\title{
ATTENTION IN DISCRIMINATION LEARNING: \\ A STIMULUS CONTROL APPROACH TO THE INTRADIMENSIONAL-EXTRADIMENSIONAL SHIFT PARADIGM
}

A dissertation presented to the Victoria University of Wellington in partial fulfillment of the requirements for the degree of Doctor of Philosophy

by

Lynne Whitney

1986 
Page No.

INTRODUCTION

THEORIES OF DISCRIMINATION LEARNING :

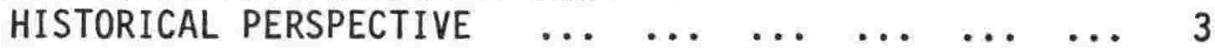

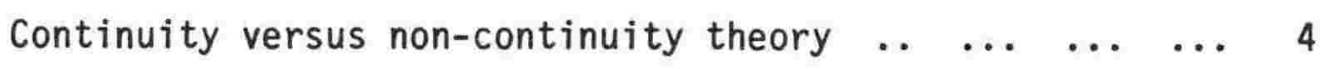

Theories of attention in discrimination learning $\ldots$... 6

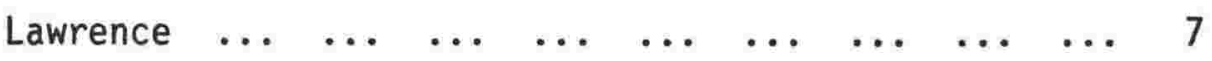

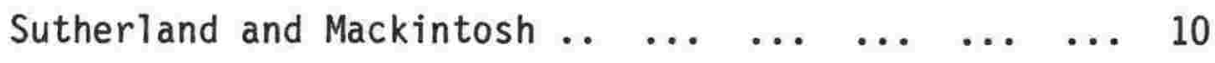

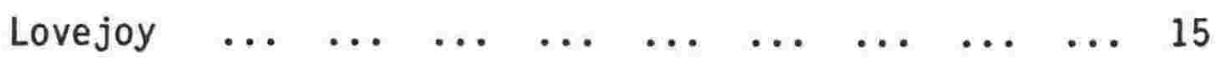

Summary of Lovejoy's (1968) and Sutherland and

Mackintosh's (1971) two-process accounts of

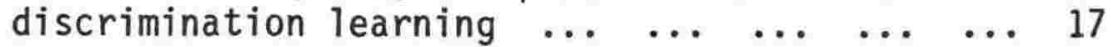

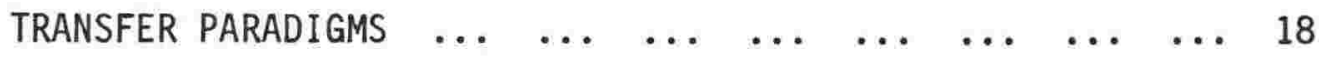

The Reversal-Extradimensional shift paradigm $\ldots \quad \ldots \quad \ldots \quad 19$

The Intradimensional-Extradimensional shift paradigm $\ldots 23$

Theoretical predictions for the ID-ED shift paradigm 27

Determinants of the ID-ED shift difference $\ldots \quad \ldots 30$

Summary of empirical results $\ldots \begin{array}{llllll}\ldots & \ldots & \ldots & \ldots & \ldots & 42\end{array}$

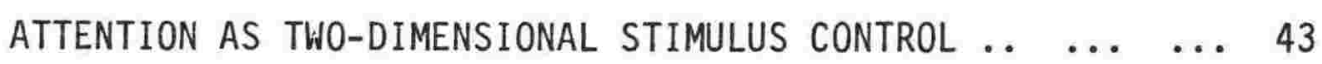

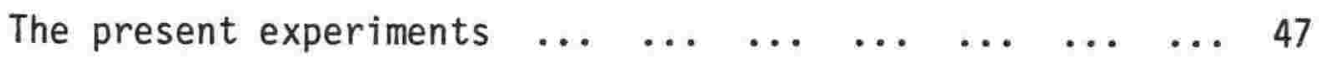

\section{EXPERIMENTS}

EXPERIMENT 1

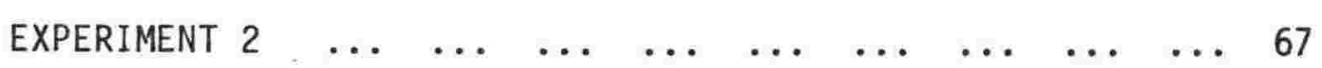

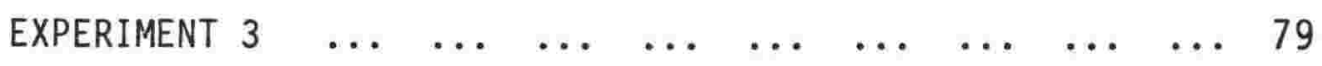

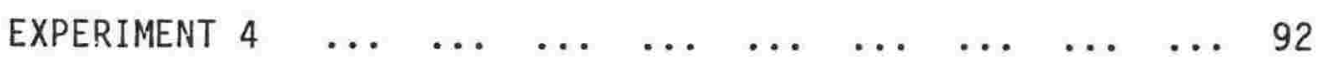


Page No.

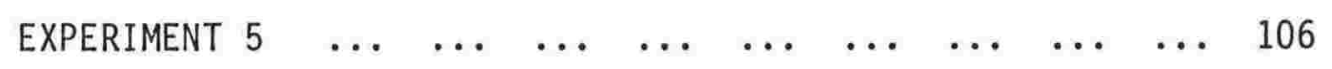

EXPERIMENT $6 \begin{array}{lllllllllll}6 & \ldots & \ldots & \ldots & \ldots & \ldots & \ldots & \ldots & \ldots & \ldots & 125\end{array}$

Comparisons of Experiments 5 and 6 .

$\begin{array}{llllllllll}\text { GENERAL DISCUSSION } & \ldots & \ldots & \ldots & \ldots & \ldots & \ldots & \ldots & \ldots & 142\end{array}$

$\begin{array}{llllllllllll}\text { Summary } & \ldots & \ldots & \ldots & \ldots & \ldots & \ldots & \ldots & \ldots & \ldots & \ldots & 147\end{array}$

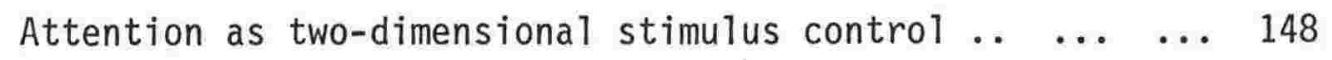

Quantification of two-dimensional stimulus control .. 149

The Chase and Heinemann (1972) Model of

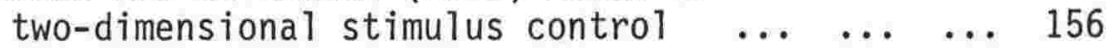

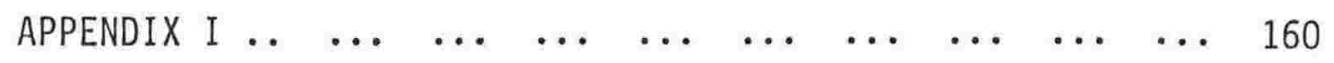

$\begin{array}{lllllllllll}\text { APPENDIX II } & \ldots & \ldots & \ldots & \ldots & \ldots & \ldots & \ldots & \ldots & \ldots & 167\end{array}$

$\begin{array}{llllllllllll}\text { REFERENCES } & \ldots & \ldots & \ldots & \ldots & \ldots & \ldots & \ldots & \ldots & \ldots & \ldots & 170\end{array}$ 
iii.

\section{LIST OF FIGURES}

Page No.

Introduction

Figure 1. Sutherland and Mackintosh's (1971)

two-process model of discrimination

learning

Figure 2. The Reversal-Extradimensional shift

Paradigm

Figure 3. The Intradimensional-Extradimensional

shift Paradigm

Experiment 1

Figure 4. Correct responses as a function of trials of Phase-2 discrimination training in the ID and ED shift

Figure 5. Two-dimensional generalisation gradients

following Phase-1: group data

Figure 6. Two dimensional generalisation gradients

following Phase-1: individual data

Figure 7. Two-dimensional generalisation gradients

following ID or ED shifts: group data

\section{Experiment 2}

Figure 8. Correct responses as a function of trials of Phase-2 discrimination training in the ID, ID (R), ED and ED (R) shift

Figure 9. Two-dimensional generalisation gradients

following Phase-1 and Phase-2 in the ID, ID $(R), E D$ and $E D(R)$ shift: group data

Figure 10. Two-dimensional generalisation gradients following Phase-1 and Phase-2 in the ID, ID $(R), E D$ and ED (R) shift: individual data

Experiment 3

Figure 11. Correct responses as a function of $8,12,16$ or 20 trials of Phase-2 training in the ID and ED shift 
iv.

Experiment 3 (cont'd)

Page No.

Figure 12. Two-dimensional generalisation gradients

following $8,12,16$ or 20 trials of Phase-2

ID or ED shift: group data

85

Figure 13. Two-dimensional generalisation gradients

following $8,12,16$ or 20 trials of Phase-2

ID or ED shift: individual data

Experiment 4

Figure 14. Correct responses as a function of trials of Phase-2 in the PD and ED shift

Figure 15. Two-dimensional generalisation gradients following 0,12 or 20 trials of Phase-2

in the PD and ED shift: group data

Figure 16. Two-dimensional generalisation gradients

following 0,12 or 20 trials of Phase-2

in the PD and ED shift: individual data

Experiment 5

Figure 17. Two-dimensional generalisation gradients following "constant-irrelevant" Phase-1 training: group data

Figure 18. Two-dimensional generalisation gradients following 12 or 20 trials of ED Phase-2 (CI-CI ,CI-2D,2D-CI ,2D-2D) : group data

Figure 19. Two-dimensional generalisation gradients following 12 or 20 trials of ED Phase-2 (CI-CI ,CI -2D , 2D-CI , 2D-2D) : individual data

Figure 20. Correct reponses as a function of 12 or 20 trials of ED Phase-2 (CI-CI,CI-2D,2D-CI or 2D-2D).

Experiment 6

Figure 21. Freeman and Thomas' (1967) training procedure

Figure 22. Generalisation gradients along the dimension of orientation following Phase-1 training for the constant-irrelevant and two-dimensional control groups 
Figure 23. Generalisation gradients along the dimension of orientation following 12 or 20 trials of Phase-2 ED shift (CI-CI,CI-2D,2D-CI, 2D-2D): group data

Figure 24. Generalisation gradients along the dimension of orientation following 12 or 20 trials of Phase-2 ED shift ( $\mathbb{C}-\mathrm{CI}, \mathrm{CI}-2 \mathrm{D}, 2 \mathrm{D}-\mathrm{CI}, 2 \mathrm{D}-2 \mathrm{D})$ : individual data

Figure 25. Correct responses as a function of 12 or 20 trials of Phase-2 ED shift (CI-CI,CI-2D, 2D-CI, 2D-2D)

General Discussion

Figure 26. A comparison of response ratings to stimulus values on the length and orientation dimensions.

Figure 27. Regression coefficients for length and orientation plotted against trials of Phase-2 training: Experiment 3

Figure 28. Regression coefficients for length and orientation plotted against trials of Phase-2 training: Experiment 4

Figure 29. Regression coefficients for length and orientation plotted against trials of Phase-2 training: Experiment 5

Appendix II

Figure 1. Two-dimensional generalisation gradients following 12 or 20 trials of Phase-2

ID (Reversal) shift: group data. 


\section{LIST OF TABLES}

Experiment 1

Page No.

Table 1. Design of Experiment 1

Table 2. Mean trials to criterion in Phase-2:

ID and ED shift groups

Table 3. Summary of ANOVARS comparing ID and ED shifts

\section{Experiment 2}

Table 4. Design of Experiment 2

Table 5. Mean trials to criterion in Phase-2:

Reversal and non-Reversal, ID and

ED shift groups

Table 6. Summary of ANOVARS comparing ID and ED, Reversal and non-Reversal shifts

\section{Experiment 3}

Table 7. Design of Experiment 3

Table 8. Summary of ANOVARS comparing ID and ED shifts over trials of Phase-2

Table 9. Summary of ANOVARS comparing ED shifts over trials of Phase-2

Experiment 4

Table 10. Design of Experiment 4

Table 11. Summary of ANOVAR comparing PD and ED shifts over trials of Phase-2

\section{Experiment 5}

Table 12. Design of Experiment 5

Table 13. F-values for ANOVARS for the eight conditions in Experiment 5

Table 14. Summary of ANOVAR to show effect of TRials, Array and Dimension over ED shift (CI-2D vs 2D-2D) 
vii.

Experiment 5 (cont'd)

Page No.

Table 15. Summary of ANOVAR to show effect of

Trials, Array and Dimension over

ED shift (2D-CI vs 2D-2D)

120

Experiment 6

Table 16. Summary of ANOVAR to show effect of

Trials, Array and Dimension over

ED shift (CI-2D vs $2 D-2 D)$

Table 17. Summary of ANOVAR to show effect of Trials, Array and Dimension over

ED shift (2D-CI vs 2D-2D)

Appendix I

Table 1. Summaries of ANOVARS to show effect of Trials of ID Phase-2 training

in Experiment 3

Table 2. Summaries of ANOVARS to show effect of Trials of ED Phase-2 training

in Experiment 3

Table 3. Summary of ANOVARS to show effect of TD or PD training in Experiment 4

Table 4. Summary of ANOVARS to show effect of Phase-1 array (CI or 2D) in

Experiment 5 ( CI-2D vs 2D-2D)

Table 5. Summary of ANOVARS to show effect of Phase-2 array (CI or 2D) in

Experiment 5 (2D-CI vs 2D-2D)

Table 6. Summary of ANOVARS to show effect of

Phase-1 array (CI or 2D) in

Experiment 6 (CI-2D vs 2D-2D)

Table 7. Summary of ANOVARS to show effect of Phase-2 array (CI or 2D) in

Experiment 6 (2D-CI vs 2D-2D) 
viii.

\section{ACKNOWLEDGEMENTS}

Geoff White's enthusiastic approach to behavioural psychology set a fine example to many students in the Psychology Department at Victoria University. I consider myself fortunate to be one of those who has benefitted from his encouragement and guidance.

I would like to thank members of staff and students in the Psychology Department for their friendship and support, and, in particular, Murray White for his assistance. I would also like to thank Rob Whitney for helping to get me underway in this research. I am indebted to the staff at the Victoria Child Care Centre, whose dedication in providing a caring 'family' for my children has made completing this work so much easier. My thanks to Christine who made such a good job of the typing.

Most sincere thanks go to Alex Skelton for his patience and support. Finally, no acknowledgements would be complete without expressing my gratitude to Lauren and Alex, who are now allowed to make as much noise as they like. 
In the present thesis, the intradimensional-extradimensional shift effect was treated as a problem of two-dimensional stimulus control. Factors determining stimulus control in the ID-ED shift were explored over six experiments. In Experiment 1 adult students were trained to discriminate between successively presented stimuli that differed in both line length and orientation. For half the subjects the length dimension was relevant (ie: different stimuli on that dimension were correlated with different outcomes) and for half the subjects orientation was relevant (Phase 1). A11 subjects were then shifted to a second discrimination between new line lengths and orientations (Phase 2). For half, this constituted an intradimensional (ID) shift in that the previously relevant dimension remained relevant; for the remaining subjects the previously irrelevant dimension was made relevant in an extradimensional (ED) shift. The ID shift required significantly fewer trials to establish strong stimulus control by the relevant dimension in Phase 2 than did the ED shift. Experiments 1 and 2 further established that such differences were not attributable to a dominance relationship between dimensions or to specific cue values.

Experiment 3 examined the development of stimulus control by the two dimensions over trials in Phase 2. In the ED shift, two-dimensional generalisation gradients showed a systematic 
weakening and strengthening of control by the Phase 1 relevant and Phase 2 relevant dimensions respectively. In the ID shift there was no change in stimulus control by either dimension. Experiment 4 established that transfer to the orientation dimension following differential training on length (ED shift) was superior to orientation following non-differential training on length (PD shift). Learning that differences on an extradimensional dimension were relevant in Phase 1 therefore had a facilitatory effect on control by orientation. Experiments 5 and 6 investigated the effects of manipulating the number of cues on the irrelevant Phase 1 dimension (orientation) and/or the irrelevant Phase 2 dimension (1ength), in an ED shift where orientation was relevant in Phase 2. Both orientation and length (Experiment 5) or orientation alone (Experiment 6) were varied in the generalisation test. The ED shift in Phase 2 was retarded by the irrelevant dimension in Phase 1.

It was concluded that in general the Phase 1 relevant dimension must lose control in Phase 2, and the Phase 1 irrelevant dimension must gain control in Phase 2 (Experiment 3). However, the inverse relation between loss of control by one dimension and gaining of control by the other does not occur in a way consistent with the Inverse Hypothesis of some selective attention theories. In addition, the previously relevant dimension in an ED shift facilitates control by the new relevant dimension in Phase 2 relative to non-differential training, consistent with attentional enhancement. The major factor found to be slowing down the 
$x i$.

development of control by the new relevant dimension in an

ED shift is the presence of the irrelevant dimension in Phase 1 , (Experiment 5). This is probably a 'learned irrelevance' effect. 
INTRODUCTION 


\section{INTRODUCTION}

In every situation requiring a discrimination, the discriminative stimuli vary along a number of possible dimensions. The dimension relevant to the discrimination is typically the one chosen by the experimenter to provide stimulus values correlated with the rules or reinforcement contingencies that operate in the procedure. Many other dimensions are irrelevant by virtue of a lack of correlation between the stimulus values they provide and the reinforcement contingencies. Some irrelevant dimensions may be arranged by the experimenter as an important part of the procedure. Others may be an inherent feature of the procedure in that the stimulus values by which they are defined remain constant ("constantirrelevant" stimuli) and afford an unchanging context for the experimental situation. A salient issue in the study of discrimination learning concerns the means by which the discriminative stimuli come to control differential responding. How does a subject learn to identify the relevant dimension? Does the subject learn to attend to the relevant dimension or ignore the irrelevant dimensions? Does the subject learn to respond to specific stimulus values of the discriminative stimuli independently of the stimulus dimensions to which they belong?

The specific question here concerns the dimensional transfer of attention. One way of examining the nature of this transfer is to use a paradigm in which a subject is trained on one discrimination and is tested on a subsequent task where either stimulus or response components are changed. The question is: which aspects of the 
stimulus array facilitate transfer and which aspects inhibit transfer to the new task?

The transfer of attention in discrimination learning has been investigated in two related forms (Hogg \& Evans, 1975). The first paradigm involves go-no-go discrimination learning followed by stimulus generalisation testing on a second, independent dimension. This is known as extradimensional (ED) generalisation (Thomas, Freeman, Svinicki, Burr \& Lyons, 1970). The second paradigm involves simultaneous discrimination learning followed by a discrimination shift where the relevant dimension of the shift phase remains the same (intradimensional (ID) shift or reversal (RV) shift) or is changed (extradimensional shift). The two types of shift are compared in terms of trials or errors to a pre-determined criterion.

The extradimensional-generalisation paradigm has been used extensively with animals (Honig, 1969, 1970: Robles, Newlin \& Thomas, 1980 : Thomas et al., 1970) and with young children (Evans \& Hogg, 1975; Hogg \& Evans, 1975: Singh \& Beale, 1978) but not with adults. The discrimination shift is the procedure most commonly used with adult humans to test attentional theories of discrimination learning (Esposito, 1975b; Harrow, 1964: Isaacs \& Duncan, 1962: Nehrke, 1973: Ozioko \& May, 1977: Shanab \& Yasin, 1979: Uh1, 1966).

The discrimination-shift paradigm was designed to test twoprocess theories of attention in discrimination learning (Fisher \& Zeaman, 1973: Lovejoy, 1966, 1968: Sutherland \& Mackintosh, 1971: 
Trabasso \& Bower,1968; Zeaman and House,1963). These theories are characterised as two-process because "they assume that the organism first engages in some form of information-processing activity with respect to the input stimuli, and then develops associations with respect to that processed information. This way of describing the process suggests a cognitive view of its nature instead of a view that refers to attentional responses" (Logan \& Ferraro,1978,p.349). Thus attention is represented as a selective, mediated process.

In general terms two-process theories of attention in discrimination learning predict flatter generalisation gradients following ED training relative to control groups for the ED generalisation paradigm, and a slower transition for the ED shift than the ID shift in the discrimination-shift paradigm. It is the aim of this thesis to integrate the two approaches by extending the ID-ED shift procedures traditionally used with adult humans to include extradimensional and two-dimensional stimulus generalisation. This thesis explores the conditions under which discrimination-shift effects occur and examines an account for the ID-ED shift in terms of different sources of stimulus control.

THEORIES OF DISCRIMINATION LEARNING:

AN HISTORICAL PERSPECTIVE

Before the early 1950 s there were two classes of theories of discrimination learning: continuity theory (Spence, 1936) and noncontinuity theory (Lashley,1929;Krechevsky,1932). Subsequent1y, the distinction between theories has become less clear, primarily because theorists have concentrated on different aspects of the 
discrimination learning process. An example of the latter strategy is seen in the case of learning to attend to the relevant dimension in theories of selective attention. This process has been variously described as a "coding response" (Lawrence, 1949), an "observing response" (Wyckoff, 1952) and "switching in a stimulus analyser" (Sutherland \& Mackintosh, 1971).

\section{Continuity versus Non-continuity Theory}

In the 1930 s the main debate in theoretical approaches to discrimination learning was the continuity versus non-continuity dichotomy. The main proponent of the continuity approach was Spence (1936) and of the non-continuity approach, Lashley (1929) and Krechevsky (1932). The major difference was in terms of the interpretation of the organism's performance in the pre-solution period of a visual simultaneous discrimination task. Lashley (1929), who represented the most extreme form of non-continuity interpretations, explained chance responding on the learning curve that preceded later learning, in terms of no learning having occurred. Spence (1936), however, argued that the learning of a discrimination was best described in terms of a cumulative process and did not agree that the absence of differential responding to stimuli on a particular dimension necessarily reflected an absence of learning about those stimuli.

Spence believed that performance in simultaneous visual discriminations could be explained without resorting to the concepts of attention suggested by non-continuity theorists. For example, in 
a simultaneous discrimination task where red is positive (correct) and blue is negative (incorrect), the stimuli can be broken down into four separate components; red, blue, left, right. The response of approaching each component has some measurable strength. The four components combine to produce compounds and on any trial the response is made to the compound with the highest "strength". Spence maintained that the excitatory and inhibitory response tendencies are directly modified by reward and did not employ any internal mediating processes. Continuity theory therefore conceptualised discrimination learning as a gradual combining of basic excitatoryinhibitory tendencies to stimulus elements. Learning could occur for any stimulus "which impinged on the animal's sensorium at or near the critical moment of response" (Sutherland \& Mackintosh, 1971, p.5).

Non-continuity theorists Lashley (1929) and Krechevsky (1932), broke away from the tradition of explaining all discrimination learning in terms of a simple combination of basic learning processes. Whereas Spence (1936) minimised the role of perceptual processing and emphasised the cumulative process of solving a problem, non-continuity theory conceptualised discrimination learning as a selective process where perceptual processing is important. In Lashley's (1929) view, attention to a dimension was all-or-none. Attention was selective to the point where, while attending to one dimension on a trial, nothing would be learned about a second dimension. This is known as the "strong non-continuity position" (Mackintosh, 1965). Learning was not, therefore, a gradual 
cumulation, but all-or-none, and involved a series of hypotheses which were modified by reward, and shifts in attention were abrupt.

The distinction between continuity and non-continuity theories in terms of their use of attentional processes was therefore clear cut, but after this period it became more "natural to characterise current theories as parallel and interactive, rather than as distinct choices" (Medin, 1976, p.132). However, these theories provided a strong foundation for new theoretical ideas. Of special interest in this respect is the work of Lawrence $(1949,1950)$ and his associates, who laid the foundation for the re-awakening of interest in the role of attention in discrimination learning.

\section{Theories of Attention in Discrimination Learning}

A number of theoretical models of attention describe and predict aspects of discrimination learning. Mackintosh (1970) believes that while there has been a proliferation of terms in this area, there are some ideas which are common to all theorists who describe learning mechanisms variously as mediational, observing, attentional or orienting responses. Such areas of common ground include beliefs that organisms "respond selectively in some not always specified manner, that all stimuli are not equally effective and that these differences in effectiveness are not merely the consequences of static variables such as discriminability or temporal relationship to the response" (Mackintosh, 1970, p.173).

Theories discussed here are those that use attentional concepts 
to characterise learning processes.

\section{Lawrence}

Lawrence $(1949,1950)$ reintroduced the role of attention in discrimination learning as a result of demonstrations of transfer effects that continuity theory could not predict. He believed two questions were of fundamental importance in the continuity/noncontinuity controversy. One involved establishing whether stimulusresponse associations were best described as involving a gradual process or were an all-or-none affair; the second, asked whether a11 aspects of the stimulus situation become associated with the response or only selected ones.

In general Lawrence's (1949) theory expresses a compromise between the original continuity and non-continuity theorists. Like the non-continuity theorists Lawrence emphasised the importance of perceptual processes and described learning as a selective process. He believed the systematic analysis of the relationship between training and test problems would provide important information about the nature of these processes. With respect to the issue of stimulus-response associations, unlike the non-continuity theories that describe shifts in attention as all-or-none or abrupt, Lawrence (1949) postulated that changes in attention are of a gradual and continuous nature. With respect to the issue of stimulus selection, Lawrence argued that any particular stimulus in a transfer task does not have a fixed salience, but rather acquires a distinctiveness as a result of its own reinforcement history and that of other stimuli 
in the situation.

Lawrence's approach "divided the usual stimulus-response association formulated by learning theorists into two parts with learning occurring within the perceptual, or 'sensory' component and within the response or 'motor' component" (1949, p.187). Learning is thus seen as a two-stage process, one involving the establishment of a coding response, the other an association between the stimulusas-coded (SAC) and the overt response. This two-stage process has continued to be an important facet of theories of selective attention (Lovejoy, 1968: Sutherland \& Mackintosh, 1971: Zeaman \& House, 1963). Sutherland and Mackintosh (1971) for example developed this idea and made it a fundamental part of their 'analyser theory'.

In his experiments with rats Lawrence (1949, 1950) aimed to analyse what is involved in learning to attend to a specific stimulus and to formulate rules which govern attentional learning. His experimental designs attempted to separate out the proposed perceptual and response components. Lawrence argued that transfer between simultaneous and successive conditional problems could not involve the transfer of any instrumental response, but would depend upon changes in the mediating attentional responses.

Using both dimensional preference and speed of transfer as his dependent variable, Lawrence $(1949,1950)$ established that positive and negative attentional effects occur, in that previous experience with a dimension (eg. brightness) in a simultaneous discrimination 
task increased the rate at which a new, successive task was performed relative to previous experience with unrelated dimensions such as width of maze or texture of maze floor. However, while establishing that such effects can occur, the experiments provided little information about the nature of the transfer effects.

Goodwin and Lawrence (1955) also examined the effect of training rats to ignore a dimension by making the dimension in question irrelevant to responding. They found that it is possible to pretrain animals to ignore a particular dimension and that such pretraining retards learning of a subsequent discrimination involving that dimension. The overall finding of Lawrence's experiments is that the rate at which a specific stimulus is learned is affected by previous exposure to that stimulus dimension and other dimensions.

Lawrence's $(1949,1950)$ experiments have been criticised on the basis of not taking into account pre-experimental dimensional preferences and on the assumption that instrumental responses required in a simultaneous discrimination are not the same as those required in a successive discrimination using the same cues (Siegel, 1967), the support for which is equivocal. Issues raised by these experiments, however, become a vital part of theories that incorporated or challenged the concept of selective attention. Lawrence's reduction of the distinction between continuity and noncontinuity theories by combining concepts from each was an important contribution to later theorising. 
Sutherland and Mackintosh

In the tradition of the non-continuity theorists (Lashley, 1929) and of Lawrence (1949, 1950), Mackintosh (1965, 1971, 1977) emphasised that it was the central process of attention that determined which stimuli impinging on the organism would control behaviour. Like Lawrence's theory, Mackintosh's two-stage (1965) theory distinguished two distinct components in discrimination learning: an attentional component and a choice component. The attentional component involved learning to attend to a relevant stimulus dimension, and determined the extent to which the subject's behaviour would be controlled by that stimulus rather than by another at that particular moment. The choice component involved the animal learning to attach the correct responses to stimuli having different values on a particular dimension.

Mackintosh (1965) saw the experimental evidence as showing that attention selects between stimulus dimensions in humans, and not between objects as orienting response theories predict. Unlike the 'strong non-continuity position' (Lashley, 1929), Mackintosh did not assume attention to a dimension to be 'all-or-none'. His modified non-continuity position assumed that attention to one cue did not preclude an animal learning anything about a second cue, but it did reduce the amount learned.

An advantage of two-stage theories over other two-stage accounts of discrimination learning is that attention theories permit the parameters associated with attentional and choice responses to be 
manipulated independently. "Having established that the amount learned about any cue can be affected by attention", Mackintosh (1965, p.131) argued "the next step would seem to be to investigate what variables increase or decrease the range of attention". One set of experiments that have tried to separate out the attentional or classification component from the choice or response component is that of 'acquired distinctiveness of cues', described earlier as formulated by Lawrence (1949). Evidence from such paradigms indicated to Mackintosh (1965) that pretraining to attend to a given dimension will benefit learning of a discrimination involving that dimension - irrespective of the relationship between the responses required in the two problems.

Sutherland (1964) was also concerned that learning theorists in the 1930 s and 1940 s had neglected the classification of stimulus imput, and had concentrated on how responses are learned. Using some of the ideas originally proposed by non-continuity theorists, his work with Mackintosh and his own work on shape recognition, Sutherland identified and named two distinct mechanisms operating in discrimination tasks. The first mechanism governed which cue or dimension the organism attended to and involved learned to "switchin analysers". The second mechanism governed the attachment of the correct responses to the outputs from the 'analyser'. Response attachments to the analyser output were said to be strengthened by reward. However, "the strength with which an analyser is switched in depends on differences in its outputs being consistently associated with the subsequent occurrence of events of importance 
to the animal, whether these events are rewarding or punishing" (1964, p.57).

Many of the ideas set out in earlier papers have been combined to form the more formal statement by Sutherland and Mackintosh (1971) of two-process theory of discrimination learning. Two major features of this model are two-process learning and the inverse hypothesis.

\section{Two-process learning.}

Sutherland and Mackintosh (1971) assume that two processes are involved in learning. Initially the organism must learn to attend to the relevant stimulus dimension, and then, learn to attach the correct responses to stimuli with different values on this dimension. These two processes operate serially, rather than in parallel. In terms of the model this involves the combined probabilities of firstly, 'switching-in' the correct analyser, and, secondly, choosing the correct response to attach to that analyser (see Figure 1). These two processes are not governed by the same rules. Responses are strengthened by reward, weakened by non-reward, but analyser strength is increased when its outputs consistently make correct predictions about further events of importance to the organism.

The inverse hypothesis.

The model assumes an inverse relationship between analyser 


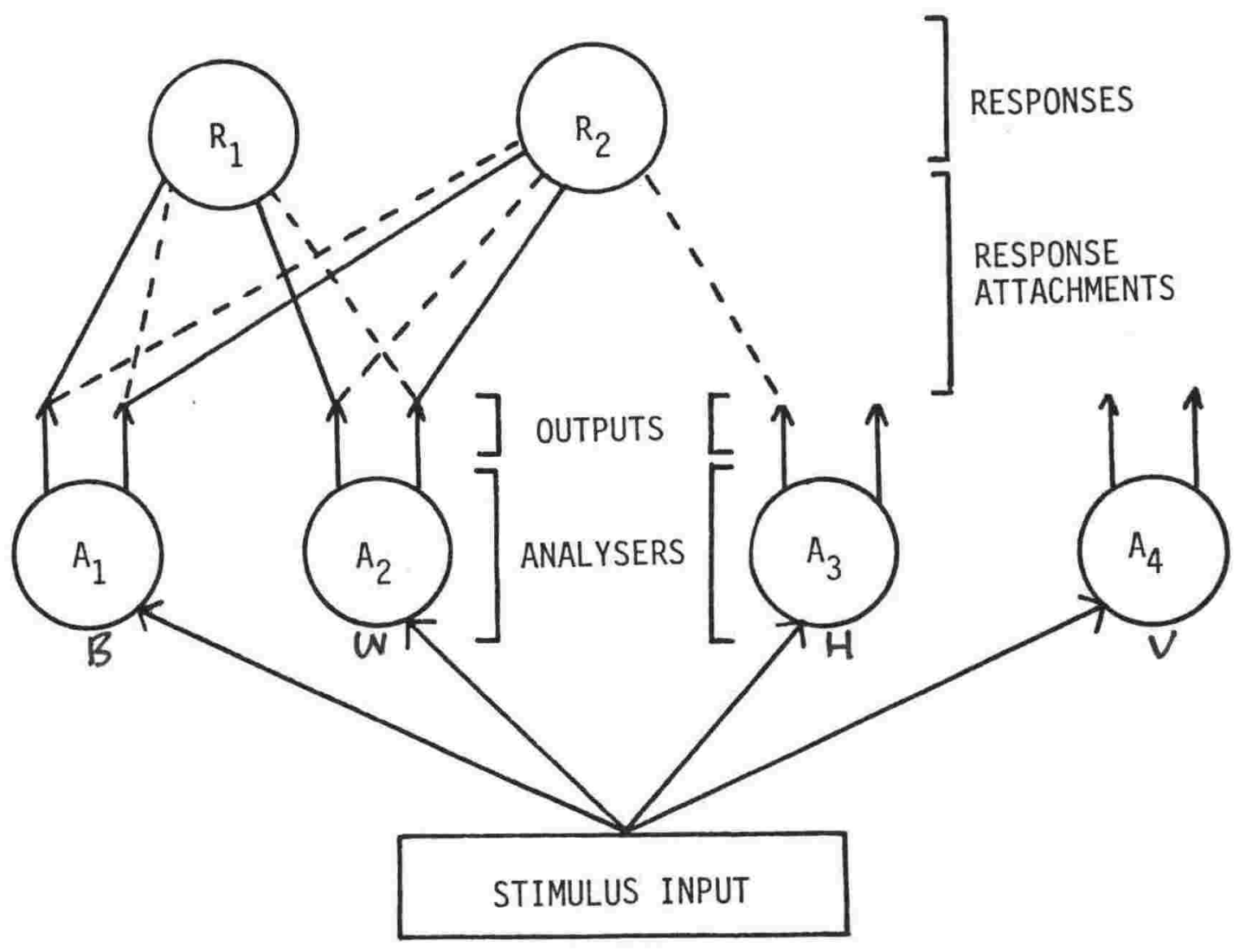

Figure 1: To show the Sutherland and Mackintosh (1971) model of two-process discrimination learning. Learned response attachments are indicated by solid lines: other possible response attachments are shown by dashed lines. B designates black; W, white; $H$, horizontal and $\mathrm{V}$, vertical. (Taken from: Sutherland and Mackintosh (1971,p.34)). 
strengths. The strengths of different analysers can be modified by procedural variables. As the probability of attending to one stimulus dimension increases, the probability of attending to the second dimension (or other dimensions) decreases in a complementary manner.

Analyser strength may be subject to direct acquisition or extinction by trial outcome, or, because analyser strengths sum to unity, may be subject to indirect acquisition or extinction. of particular interest in this respect is the assumption that the relative dominance of different analysers can be affected by different training procedures. Therefore, the way the organism learns about the dimensions in a discrimination task can be modifed by the training procedure itself.

Mackintosh (1965) commenting on results from Goodwin and Lawrence (1955), recognised that it was possible to show that prior training with a cue that is irrelevant retards subsequent learning when that cue is relevant. Irrelevance is defined experimentally when two cues on a given dimension are non-differentially reinforced. Sutherland and Mackintosh (1971) showed that "discrimination training depresses subsequent control of behaviour by irrelevant stimuli present during training" (p.467), and that differential training on a salient rather than a non-salient dimension suppresses a new discrimination. 
Lovejoy

Lovejoy (1968) described a quantitative theory for two-choice discrimination learning, primarily designed to explain simultaneous visual discriminations by rats. Like Sutherland (1964) and Mackintosh (1965), Lovejoy (1968) claimed that simultaneous discriminations should be characterised in terms of stimulus rather than response selection.

Lovejoy's (1968) model III draws a clear distinction between two selective mechanisms which are normally described together as 'selective attention'. The first of these is a control mechanism, which determines how an animal responds selectively to one dimension or another. It is what Mackintosh (1965) refers to as learning to attend to the relevant stimulus dimension. A control strength exists for each dimension and is determined by the relationship between the distinctiveness (or salience) of the dimension and by cue-specific feedback expressed in terms of the preference for the two cues on that dimension (see below). The second of these is a learning mechanism, and determines how an animal learns about one dimension or another.

In examining the relationship between these two mechanisms, Lovejoy assumes, unlike Sutherland and Mackintosh (1971) 's 'inverse hypothesis', that animal's "simply learn about that one cue by which they were just controlled" (1968, p.45). Evidence has shown that this 'strong non-continuity assumption' is not always correct. Lovejoy modified this assumption to some extent, but suggested there 
is evidence to suspect a correlation between control and learning. Firstly, both the probability of control by a dimension in his Model III and the probability of learning about a dimension depend primarily on the distinctiveness of that dimension. However, distinctiveness is not the only factor that determines which dimension controls behaviour. The probability of a dimension controlling behaviour is defined by:

$$
\frac{D_{i}+\Delta_{i}}{V(1-V)}
$$

where $D_{j}$ in the nondirectable and $\Delta_{j}$ the directable component of a cue's distinctiveness, and $V(1-V)$ represents the preference for two cues on the dimension. Where only one cue is available on a dimension (ie: constant-irrelevant), this feedback principle predicts that at the end of training the probability of control by that dimension remains at its base-level distinctiveness or salience. The control and learning mechanisms can therefore be modified by prior experimental experience.

Evidence has shown that pre-training with a cue can facilitate or inhibit future learning or control with that or other cues (Lawrence, 1949: Mackintosh, 1965). "Such training makes an animal more likely to attend to the previously relevant cue, and hence less likely to attend to other cues" (Lovejoy, 1968, p.57). Lovejoy uses attention for situations where it is known that learning or control is changing, but we cannot specify which, and he reserves this term in his own model for when control and learning are perfectly correlated. 
Summary of Lovejoy's (1968) and Sutherland and Mackintosh's (1971) Two-process accounts of Discrimination Learning

Lovejoy (1968) and Sutherland and Mackintosh (1971) offered two-process accounts of discrimination learning. The first of these processes involves learning to attend to the relevant stimulus dimension and the second process involves learning to attach the correct response to stimuli on that dimension. In both accounts the two processes are described as operating serially, rather than in parallel, and the first process is an attentional, dimension-specific factor.

In both models learning is conceptualised as a gradual process, incrementing over trials. The theoretical postulates of acquisition and extinction implicated in learning, and that correspond to the operations of reinforcement and non-reinforcement in discrimination learning, are applied to both the first and second stages of both models in some form. In particular, with respect to the first, attentional stage, acquisition processes operate directly to increase the probability of attending to the relevant dimension of training, while extinction processes operate to decrease the probability of attending to the irrelevant dimension.

Both Lovejoy, and Sutherland and Mackintosh subscribe to an "inverse hypothesis" of attentional modifiability. As the probability of attending to one dimension increases the probability of attending to all other dimensions decreases in a complementary way. 
An important aspect of these theories for this thesis is how to account for changes in attention that result from different training procedures. This raises the issue of to what extent the theories allow for modifiable as opposed to non-modifiable aspects of attention. "An aspect of attention is called modifiable, or directable, insofar as the response strength or sampling probability for a particular dimension (or a given set of cues) may change with reinforcement. It is non-directable if it remains constant for a given set of cues throughout training" (Fisher \& Zeaman, 1973, p.205). Theoretical descriptions vary as to the extent they allow for modifiable aspects of attention. Lovejoy (1968) has made this distinction explicit; Sutherland and Mackintosh (1971) acknowledge that attributes of the incoming stimulus (eg. stimulus novelty) can also determine analyser strength, as well as changes in strength due to learning.

Both Lovejoy, and Sutherland and Mackintosh acknowledge the important role of the irrelevant dimension in Phase 1 in determining the degree of control by that same dimension in Phase 2. For example, Lovejoy (1968) would predict that where only one cue is available on the irrelevant dimension in Phase 1 (constant-irrelevant), at the end of training the probability of control by that dimension would remain at its base-level salience, rather than being reduced to virtually zero. 
paradigms where a discrimination with different stimulus dimensions is transferred to a new discrimination. The general rationale is to change the dimensions or specific training values in the transfer task in order to determine their relative importance. The two transfer paradigms described below are the Reversal-Extradimensional shift (RV-ED) comparison and the Intradimensional-Extradimensional shift (ID-ED) comparison. These paradigms have been specifically used to test some of the theoretically critical issues in accounts of two-process theories of attention in discrimination learning as will be explained later. For example, they provide the opportunity to assess the importance of prior experience in determining dimensional salience, the effects of changing stimulus attributes in dimensional transfer, and can differentiate between theoretical positions with respect to the manner in which dimensions are processed.

\section{The Reversal-Extradimensional Shift Paradigm}

The Reversal (RV) shift and Extradimensional (ED) shift comparison was introduced by Buss (1953) and received a great deal of theoretical and methodological examination. The shift comparison took a number of forms according to the nature of the stimulus material, the pre-shift and post-shift array, but a typical RV-ED shift comparison would involve the following basic design (Figure 2). In the pre-shift discrimination task, one dimension (colour) is designated 'relevant' and the other dimension (form) 'irrelevant' to the solution of the problem. The relevant dimension is consistently associated with reinforcement, while the irrelevant dimension is reinforced randomly. After a training criterion has 
$\underline{\text { PHASE - } 1}$

(Training Stimuli)

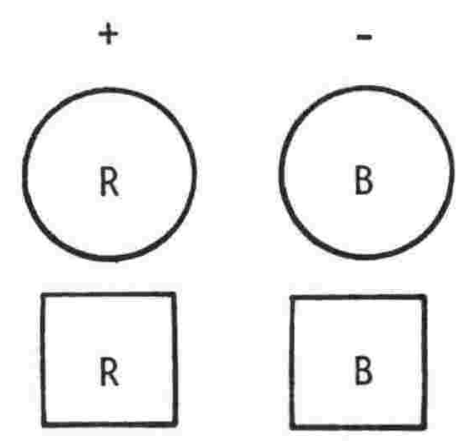

PHASE - 2

(Transfer Stimuli)

REVERSAL

SHIFT

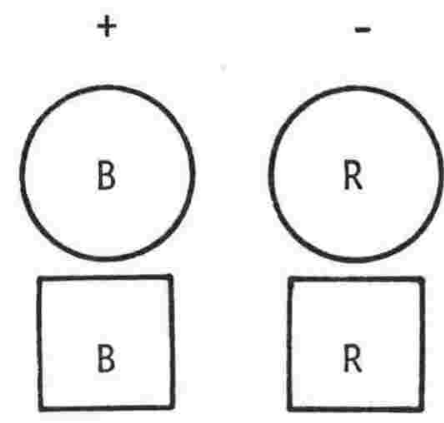

EXTRADIMENSIONAL SHIFT

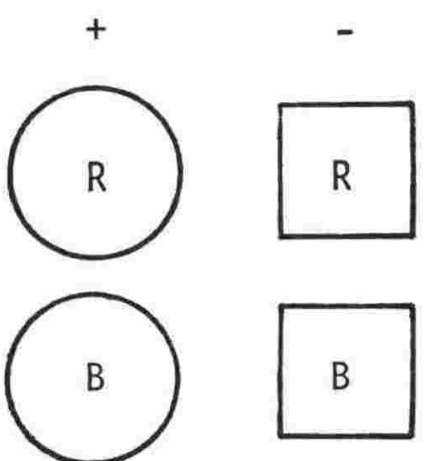

Figure 2: To show a typical Reversal versus Extradimensional Shift paradigm, using colour $(R=$ Red; $B=B l u e)$ and form dimensions. 
been reached, subjects are transferred, usually without any prior warning, to a RV shift or an ED shift (Figure 2). The RV shift requires the subject to continue to respond to the previously 'relevant' colour dimension, but responses that were reinforced in the presence of red are now reinforced in the presence of blue. The stimuli are identical for the ED shift, but now reinforcement is associated with the previously 'irrelevant' dimension - form, and colour is reinforced randomly.

Using a block classification task, Buss (1953) found that subjects performed an RV shift faster than a non-RV shift, although both were performed more slowly than the original pre-shift discrimination task. He accounted for this difference in terms of partial reinforcement. He claimed the acquisition of the new response in the ED shift condition was retarded by the intermittent reinforcement of pre-shift responses to stimuli during the post-shift phase. For example, a response to red (Figure 2) in the post-shift ED condition was still reinforced on $50 \%$ of the trials, as opposed to $100 \%$ on pre-shift trials, even though it was no longer relevant to the correct solution of the problem. This served to retard the extinction of responses to the old relevant dimension, colour. In the RV shift, however, the reinforcement contingencies were completely changed and old responses were never reinforced, hence no delay in extinction. Thus performance was poorer in the ED shift compared to the RV shift.

Kendler and D'Amato (1955) repeated the RV-ED shift in a card 
sorting task, but they designed the post-shift task so that there was no opportunity for partial reinforcement in the first few post-shift trials. There was still a performance decrement in the ED shift, which could not be accounted for by partial reinforcement.

The RV-ED shift comparison was the most popular means in the 1950s and early 1960s of testing whether two-stage theories (eg. Lawrence, 1949: Zeaman \& House, 1963) or single-stage theories (eg. Spence, 1936) could best account for the transfer of various responses in discrimination tasks. The main problem with the RV shift was that it incorporated in the design two types of response transfer. Shepp and Turrisi (1966) refer to these as instrumental-response transfer and mediating-response transfer. Mediating-response transfer refers to the transfer of such conceptual responses as the activity of 'stimulus analysers' (Sutherland \& Mackintosh, 1971), and observing responses (Wyckoff, 1952: Zeaman \& House, 1963). The nature of this response forms the major difference between the number of two-stage mediational theories that have been invoked to account for discrimination learning. Wolff's (1967) review of the ID-ED paradigm concluded that the "principal factors operating in the shift process in general are probably attentional in nature, as Zeaman and House (1963) have proposed, rather than verbal or perceptual as other investigators have sometimes implied". Opportunities for instrumentalresponse transfer are found in situations where specific stimuli present in the pre-shift discrimination task are present in the postshift task. This occurs in the RV shift where the stimuli are not changed from one task to the other (see Figure 2), but response 
contingencies are changed.

The availability of instrumental-response transfer posed problems for the RV-ED shift. Slamecka (1968) evaluated the development of shift paradiams and their theoretical implications for accounting for human visual discriminations. He examined the RV-ED shift in detail and discussed five sources of bias associated with this paradigm for testing single stage versus two-stage theories. Slamecka described three sources of bias which operated in favour of a faster solution of the RV shift: the differential opportunity for detection of shifts, the differential presence of intermitcent reinforcement and, the differential obviousness of post-shift solutions (1968).

These methodological problems, together with the differential transfer of sorting responses in the RV-ED shift made results difficult to interpret with respect to single versus two-stage theories of attention. slamecka (1968) therefore believed the tactic was to "abandon the use of the RV shrft while maintaining fidelity to the original shift rationale and to the theoretical question that its use was intended to answer" $(p .435)$. He sought to replace it with a new paradigm that had been reported by Eimas (1965) as more appropriate for examining the role of attention in discrimination learning - the Intradimensional (ID) - Extradimensional (ED) shift comparison.

\section{The Intradimensional-Extradimensional Shift Paradigm}

The ID-ED comparison involves two phases of discrimination training. A typical ID shift design involves training a subject to 
discriminate between stimuli which may be presented successively or simultaneously. The training stimuli vary along two dimensions (Figure 3). As in the RV-ED shift, one dimension is designated 'relevant' and the other dimension 'irrelevant' to the solution of the discrimination task. One component of the relevant dimension is consistently associated with reinforcement, while the other component on this dimension is associated with either no reinforcement or reinforcement at a lower rate. The second dimension remains irrelevant and is reinforced randomly throughout the training task. The subsequent post-training shift involves testing the subject with new stimuli which are made up of two new values from the same two dimensions. Differential reinforcement in the second phase of an ID shift is still associated with the original 'relevant' dimension of the first phase.

The ED shift uses identical stimuli to the ID shift in both the training and the post-shift phases. Training procedures follow the same course as those given for the ID shift. In the second phase, however, differential reinforcement is associated with the previous irrelevant dimension and the previous relevant dimension is now randomly reinforced. In terms of Garner's (1970) distinction between dimensions and features, in the ID-ED shift, both dimensions remain the same in the pre-shift and post-shift phases, whereas features (values of stimuli on the dimensions) change between the phases. In the RV-ED shift, both dimensions and features remain the same between phases.

One advantage of the ID-ED over the RV-ED in comparing different 
PHASE - 1

(Training Stimuli)

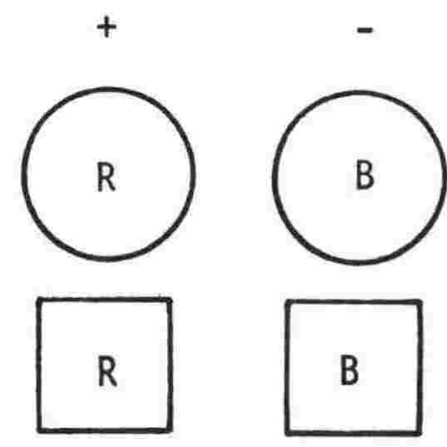

PHASE- 2

(Transfer Stimuli)

INTRADIMENSIONAL SHIFT

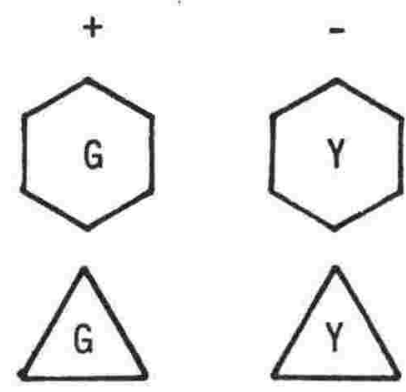

EXTRADIMENSIONAL SHIFT

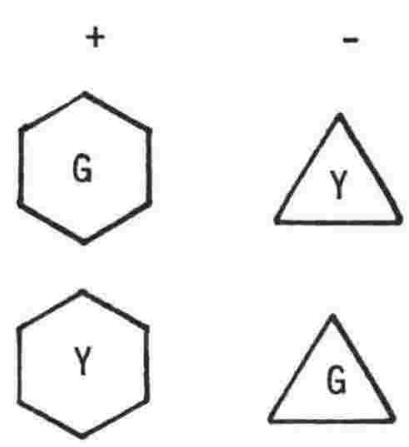

Figure 3: To show a typical Intradimensional versus Extradimensional Shift paradigm, using colour $(R=$ Red; $B=B l u e ; G=$ Green; $Y=$ Yellow) and form dimensions. 
theoretical perspectives is that it reduces the comparison to a theoretically critical one: what conditions affect the post-shift dimensional change. A second advantage is that it eliminates possible partial reinforcement effects due to instrumental-response transfer, while still allowing for attention to transfer. That is, positive attentional transfer can be assessed in the absence of instrumental-response transfer. In Lawrence's experiment (1949), instrumental-response transfer was eliminated, and by retaining the same stimuli for both the ID and ED shift in the pre-shift and postshift phases the same basic manipulation is achieved. Only the reinforcement contingencies associated with those dimensions change.

Experiments utilising the ID-ED shift or 'total change' paradigm (Esposito, 1975a) were first reported by Shepp and Eimas (1964) with rats and later with human subjects (Shepp \& Turrisi, 1966: Trabasso, Deutsch \& Gelman, 1966) although the paradigm has been included in earlier experiments under other names. For example, Isaacs and Duncan (1962) incorporated such a comparison in their experiments calling them "Non-Reversal to the Same dimension (NRS)' (cf. ID shift) and 'Non-Reversal to Different dimension (NRD)' (cf. ED shift). They were the first to include an ID-ED shift with adults in their experimental design. They noted that there was disagreement among various researchers (Buss, 1953: Harrow \& Friedman, 1958: Kendler \& D'Amato, 1955) who had used the RV-ED shift to establish whether a mediational theory could account for the way human adults learn and transfer in such concept tasks. Isaacs and Duncan (1962) concluded that failure to confirm unambiguously the theoretical predictions 
could be avoided if appropriate control groups were provided. As mediation theory would predict (Kendler \& D'Amato, 1955) human adults consistently took more trials to learn non-Reversal shifts than Reversal shifts. However, mediation theory, which utilises the transfer of a dimension-specific response, also predicted that the RV shift should be learned faster than a control group that learns only the transfer task, because the reversal group should allow for the positive transfer of that dimension-specific response. It was here that the ambiguities arose. The superiority of the Reversal group in terms of trials to criterion over a control task only group, depended on the nature of that control group.

For example, Isaacs and Duncan (1962) found that the RV condition had significantly fewer trials to criterion $(\bar{X}=7.52)$ than an ED shift condition $(\bar{X}=13.77)$. It was also found that an ID shift condition $(\bar{X}=3.03)$ was performed the fastest. From these results they concluded that performance in all groups could largely be accounted for by a combination of two factors: "non-specific transfer (eg. a warm-up effect) and a specific tendency to continue to respond in transfer to the dimension of stimuli reinforced in training" ( $p .585)$. The ID-ED shift paradigm is still being used to investigate the conditions under which attention can be modified.

\section{Theoretical predictions for the ID-ED Shift Paradigm}

Two-process theories of attention in discrimination learning predict that in general the ID shift will be performed faster than the ED shift because the dimension-specific first stage means that 
28.

attention will transfer more rapidly within than between dimensions. The theories describe the development of some cue-independent strength of attention. Acquisition processes in the first discrimination task of the shift (Phase-1) increase the probability of attending to the relevant dimension, and decrease the probability of attending to the irrelevant dimension. This irrelevant dimension subsequently becomes relevant in the second discrimination task (Phase-2) only for the ED shift.

The 'inverse hypothesis' demands that as the probability of attending to the relevant dimension in Phase-1 nears 1.0 , the probability of attending to the irrelevant dimension is virtually zero. The ED shift will therefore be performed relatively slowly because the probability of attending to the relevant dimension in Phase-2 is low and the process of re-learning the relevance of this dimension is seen as a gradual one. The ID-ED comparison itself is not designed to test the correctness of the inverse hypothesis as the procedure requires the subject to respond to one dimension at the expense of the other. It is important, however, to note how such a parameter affects the predicted performance of the two types of shift.

Sutherland and Mackintosh's (1971) model specifically predicts that "discrimination training suppresses subsequent control of behaviour by irrelevant stimuli present during the training" (E-31, p.457). They hypothesise that if a dimension is present but irrelevant in Phase-1, subsequent learning about that dimension in Phase-2 is impaired compared to the case where the dimension is not 
present in Phase-1. This would suggest that the positive transfer would be partly a result of the neutralising of irrelevant stimuli during Phase-1 training.

An alternative to not presenting the Phase-2 relevant dimension in Phase-1 is to hold the cue on the dimension constant. In other words, the cues on the relevant dimension of Phase-1 are permitted to vary and are associated with differential reinforcement, while the cue on the irrelevant dimension of Phase-1 remains at a constant value. It has been argued that the probability of attending to a dimension is directly related to the physical distance between cues on a dimension. Fisher and Zeaman predict that when the irrelevant dimension is held constant-irrelevant "with no competition from irrelevant dimensions the remaining relevant dimensions (with variable cues, of course) control performance to about the same extent in both ED and ID conditions" (1973, p.220). The control strength mechanism in Lovejoy's (1968) model predicts the same ID-ED performance when the irrelevant dimension of Phase-1 is constant-irrelevant.

Sutherland and Mackintosh (1971) modified their earlier prediction (E-31) of the effects of discrimination learning to take the salience of the dimensions into account (E-25). While nondifferential training of a salient dimension may retard subsequent learning on that dimension, non-differential training with a nonsalient cue may facilitate subsequent learning. As Sutherland and Mackintosh's (1971) model includes both non-modifiable, fixed, and modifiable components, the model would therefore predict some 
interactions between dimensional preferences and the type of shift.

In summary, the main prediction for the ID-ED shift paradigm by two-process theories discussed here is that the ID shift will be performed faster than the ED shift. However, two main factors are described that qualify this prediction. Firstly, the effects of ID and ED training may be modified by the presence, absence or constant-irrelevance of the Phase-2 relevant dimension in Phase-1. Secondly, by the pre-experimental salience of the dimensions.

\section{Determinants of the ID-ED Shift Difference}

Studies of the performance of human visual discrimination tasks have followed two basic strategies. "The first has been concerned with the testing of hypotheses regarding the properties of mediating responses and the attempt to discover process laws pertaining to these responses. The second class of experiments has been either developmentally or comparatively oriented (Shepp \& Turrisi, 1966, p.86). With regard to the developmental question, Esposito (1975a) reviewed the experimental variables that affect children's performance on discrimination shift tasks. The theoretical issue around which his paper was organised was "whether or not young children can mediate or conceptualise stimuli as being instances of more general dimensions" (p.432) and at what age mediation begins. Kendler and Kendler (1962) proposed a developmental trend in the ability to exhibit a mediated response. This question has been examined empirically by the comparison of various types of transfer task. It is the ID-ED shift which is relevant to the present review. 
A. number of studies (eg. Nehrke, 1973: Shanab \& Yasin, 1979: Uhl, 1966) have examined the effects of manipulating different variables on the performance of the ID-ED shift. Results from these experiments have shown that ID shifts are performed faster in terms of trials or errors to a pre-determined criterion than are ED shifts. Wolff (1967) concluded that perhaps the "best established finding in the CST literature is that for college students in twochoice tasks, ID are easier than ED shifts - at least in the absence of specific task instructions" (p.377), and this pattern of results continues to be consistently found (eg. LeBow \& Tritt, 1971: Shanab \& Yasin, 1979).

\section{Dimensions.}

In visual, two-choice discriminations, the concept of stimulus dimension is critically important. Shepp and Turrisi (1966) proposed that the concept of stimulus dimension is useful as an operational method of specifying stimulus arrays, and also as a theoretical construct along which transfer may be postulated to operate. A stimulus dimension is defined operationally as a class of stimuli (Shepp \& Turrisi, 1966, p.87) and comprises two subcategories. The first subcategory, relevant versus irrelevant dimensions, has been discussed with respect to the shifts described earlier. Relevant dimensions are where stimulus values on the dimension are correlated with different reinforcement, while irrelevant dimensions are those where dimensional stimulus values are related randomly to reinforcement. The second subcategory, involves a qualitative versus quantitative distinction. Qualitative dimensions have no 
immediately obvious underlying continuum, but may be multidimensional (eg. form), while quantitative dimensions allow continuous variation in stimulus value (eg. size). The contribution of the effects of the different combinations of stimulus dimensions has been investigated in the fairly limited literature of adult ID-ED shift comparisons. In particular, the properties of stimuli and stimulus dimensions has been wel1 researched (Esposito, 1973: Harper, 1978: Harrow, 1964: Kennedy \& Gersten, 1976: Ozioko \& May, 1977: Uh1, 1966: Watson 1975).

\section{(i) Dimensional salience}

One important property of stimulus dimensions is their relative salience (Lovejoy, 1968). Harrow (1964) claimed that "in order to relate the results in this area to various theoretical formulations with greater exactness, there is need of a more precise analysis concerning which stimulus factors or combination of factors, are responsible for the relatively quicker learning of reversal shifts by most older human subjects" (p.330). Although this specifically related to the reversal-ED shift, the same also applies to the ID-ED shift. Harrow (1964) counterbalanced his dimensions in the ID and ED groups. Half the groups transferred from a colour to a shape discrimination, the other half from shape to colour. Comparing Harrow's ID group to the ED group it was found that in both cases (ie. shape to colour and colour to shape) that the ID was performed significantly faster than the ED. However, there was also a significant interaction (dimension by shift) in the relevant ANOVA. For the shape to colour groups the difference in performance was mean trials to criterion of 2.3 and 6.4 for the ID and ED groups 
respectively. For the colour to shape groups, however, mean trials to criterion were 4.4 and 38.4 for the ID and ED groups respectively. These data show that the shape discrimination took more trials to learn than did the colour discrimination, particularly in the ED shift condition. The nature of the specific dimensions must therefore influence the extent of difference between ID and ED shift conditions.

Dimensional salience was also demonstrated by Ozioko and May (1977) using adult college students as subjects. Preliminary scaling of the five dimensions (form, number of forms, size, position and dots within the form) by subjects who did not participate in the main experiment, showed form to be the most dominant dimension and position to be the least dominant. Using errors to criterion as the dependent variable, Ozioko and May (1977) showed two main effects of dimensional dominance: (1) In Pre-shift training, those groups for which the most dominant dimension was relevant made significantly fewer errors (1.88) than those groups for which the least dominant dimension was relevant (7.00 mean errors). (2) In Post-shift transfer there was no significant difference between ID and ED performance (n.s.) when the dominant dimension was relevant in Phase-2. But, ID was learned significantly faster than the ED when the least dominant dimension was relevant.

In an experiment using university students, Esposito (1973) demonstrated that the ID-ED difference could be attenuated by using a highly salient or dominant dimension. He examined the combined effect of dimensional salience and novelty of stimuli in a design 
which compared ID and ED shifts using partial and total change designs. The dependent variables were number of transformed trials and number of errors to criterion. He found that for the total change low saliency group there was a significant difference between ID and ED shift performance, whereas for the total change high saliency group this difference did not reach significance. Esposito (1973) also established in this experiment that the total change (ID-ED) paradigm was superior to the partial change design for examining shift learning as in the latter, novelty effects are confounded with shift effects. The results of experiments using partial change designs may therefore be due to stimulus novelty rather than attentional processes.

Dimensional dominance has been shown to interact with other effects. Esposito (1975b) examined the effect of dimensional dominance and overtraining on the performance of the ID-ED shift with adult students. His dependent variable was mean number of trials to criterion. Esposito found that overtraining facilitated the ID shift for the low-dimensional salience group, but not the ED shift. Overtraining had no differential effect on the high-dimensional salience group. This had the effect of increasing the ID-ED difference in the low-salience group compared to the high-salience group. This result extends Campione's (1969) findings with retardates that the ID-ED difference may increase when the shift is to a non-preferred or nonsalient dimension, to a case where overtraining is included. However, in the criterion-trained group (Esposito, 1975b) there was no difference between performance on the ID and ED shifts for either 
high or low saliency groups, but the high-saliency groups performed both tasks significantly faster than the low-saliency groups.

These studies (Esposito, 1973, 1975b: Harrow, 1964: Ozioko \& May, 1977) therefore demonstrate the importance of examining the effects of dimensional salience or dominance in an ID-ED shift paradigm in order that differential performance can be attributed to the manner in which attention transfers from one task to another, rather than accounted for by other dimension related factors.

\section{(ii) Nature of the stimulus dimension}

One branch of enquiry has investigated the qualitative nature of the stimulus dimensions used. Eimas (1966) attempted to support a dimensional mediation hypothesis that predicted superior performance in an ID shift compared to an ED shift using a total change design. He found that the ID shift was performed significantly faster. Commenting on the Eimas (1966) use of geometric stimuli, Slamecka (1968) suggested that 'the usual colored geometric stimuli ... employed in this experiment might conceivably allow a non-mediational account based upon the operation of a primary stimulus-generalisation mechanism' (p.436).

In his own study, Slamecka (1969) used a total change (ID-ED) paradigm with symbolic stimuli from various different "categorical dimensions" (eg. animals, sports, cars, chemical, cities). Slamecka (1969) found no pre-shift effect and that the post-shift performance on the ID shift was superior to that on the ED shift. 
LeBow and Tritt (1971) argued that Slamecka's (1969) dimensions may not have been comparable with traditional dimensions such as geometric form, colour, number and they repeated the experiment using typed words. An example of their stimuli would read "blue two squares" or "sma11 centre warm". Again there was no pre-shift effect, but the ID shift was performed more accurately than the ED shift. Both Slamecka (1969) and LeBow and Tritt (1971), however, had used control groups which shifted to an entirely new set of dimensions, and, whereas Slamecka had found significant positive and negative transfer effects for the ID and ED groups respectively when compared to the control, LeBow and Tritt did not. One of the conclusions that LeBow and Tritt drew from this discrepancy was that "variables such as type of stimuli and number of dimensions used.... may be important in concept shift experiments" (1971, p.327) and may determine whether or not a significant ID-ED performance difference is obtained.

Kennedy and Gersten (1976) examined transfer with geometric stimuli (eg. form: circle-triangle) and symbolic stimuli (with corresponding forms written in black, eg. ONE BLUE CIRCLE). Kennedy and Gersten found no pre-shift effect. The post-shift mean trials to criterion were 0.44 and 1.27 for ID and ED shifts respectively using geometric stimuli, and 0.67 and 1.17 for ID and ED shifts using symbolic stimuli. Of particular interest is the absence of a Shift by Stimulus type interaction. Kennedy and Gersten concluded that "this finding lends no support to the hypothesis that stimulus generalisation may be a biasing factor favouring ID shift performance with coloured geometric stimuli as proposed by Slamecka 
(1968)" (1976, p.460).

Particular stimulus values on the dimensions chosen for the concept-shift tasks, however, may be important. Using college students, Uhl (1966) compared the performance of an ID shift and ED shift when the similarity between training and transfer stimuli was systematically varied. Uhl found an interaction between type of shift and shift stimulus. In Phase-1, all stimuli were coloured forms $(F-V)$ that also varied in size and position. In Phase-2 the stimuli were now coloured forms (F-V - identical or GW - different). In ID groups form was relevant dimension throughout, while in ED groups subjects transferred from a form to a colour discrimination. Identical stimuli in training and transfer arrays produced a slower ED shift and a faster ID shift relative to the case where different stimuli were used. Uhl (1966) concluded that the mediating response was weakened by generalisation decrement. This weakening of the mediating response makes the ID shift more difficult and the ED shift easier. The results of this study "indicate that more refined analysis in terms of stimulus attributes should be part of experiments that compare IN (ID shifts) and EX (ED shifts)" (Uh1, 1966, p.433).

\section{Variability of the irrelevant dimension.}

In a simultaneous discrimination the irrelevant dimension can be varied within trials, between trials or be held constant, and in a successive discrimination can be varied over trials or held constant (constant-irrelevant). Esposito (1975a) concluded that this change in presentation of the irrelevant dimension had a 
consistently strong influence on the relative rates of ID and ED shifts in children. Specifically, in a simultaneous paradigm results showed faster ID transfer than ED transfer when the irrelevant dimension was varied between trials.

Dickerson, Wagner and Campione (1970) varied the irrelevant dimension in the post-shift task only, while in a separate study Shepp and Gray's (1971) factorial design included such manipulations in both the pre-shift and post-shift task. The manipulation in both studies involved varying the presentation of the irrelevant dimension within and between trials. The general result, in agreement with Esposito (1975a) showed that when the irrelevant dimension was varied within trials in the post-shift task, children learned the ED shift slower than the ID shift, but this difference was eliminated when the irrelevant dimension was presented between trials.

Ozioko and May (1977) investigated the effects of irrelevant dimension variability as studies by Dickerson et al. (1970) had done. This was to see whether Esposito's (1975a) general finding could be replicated with adult subjects. Ozioko and May examined two factors. Firstly, the irrelevant dimension could vary either within or between trials of a simultaneous discrimination, in both pre-shift training and post-shift testing. Secondly, (as discussed above), the dominance of the stimulus dimensions was examined.

The results of Ozioko and May's (1977) experiment showed all three main effects (type of shift, dimensional dominance and 
variability of the irrelevant dimension) to be highly significant. The college students therefore learned the ID shift faster than the ED shift when the irrelevant dimension varied within trials. The ID-ED shift difference was not significant when the irrelevant dimension varied between trials. These results also demonstrated the importance of taking the relative dominance of the dimensions into consideration as the ID-ED difference only occurred when the post-shift array had a low-dominant dimension relevant. Ozioko and May (1977) believe that their results extend the findings of Dickerson et al. (1970) to adults and conclude "the negligible difference between types of shift under BETWEEN-trial variation may be due to the non-competitiveness or WITHIN-trial constancy of the irrelevant dimensions" (1977, p.316).

Trabasso, Deutsch and Gelman (1966) working with children, varied the presentation in another way. In attempting to assess the relative roles of attentional and instrumental responses they incorporated the ID-ED shift comparison and used single dimensional rather than two-dimensional problems. They argued that "methodological and theoretical considerations that result from partial reinforcement both during initial training and shift learning are circumvented by the absence of the irrelevant dimension" (p.12). There was no difference between mean error for ID and ED shift when no irrelevant dimension was present. It therefore appears to be important to examine the influence of the irrelevant dimension, particularly in those cases where the manipulation of the irrelevant dimension leads to an absence of the typical ID-ED shift difference. 
40.

Buss (1953) had earlier suggested that partial reinforcement was a factor in the differential performance of discrimination shift tasks. He argued that the Reversal shift was performed faster than the ED shift because partial reinforcement (in this case $50 \%$ ) of the irrelevant dimension during training in the ED shift impaired transfer to that dimension when it became relevant during the transfer phase. Subsequent experiments involving various types of discrimination transfers have shown the relationship between partial reinforcement effects and performance to be complex (Abraham et al, 1964: Gormezano \& Grant, 1958: Harpur, 1968).

\section{Type of solution mode.}

Lowenkron (1975) investigated the idea of conceptual versus non-conceptual modes of learning. Subjects were identified as conceptual (consistent) responders or non-conceptual (inconsistent) responders on the basis of their responses to non-outcome trials in both acquisition and transfer phase. "Conceptual" subjects responded on no-outcome trials in a manner consistent with the reinforced contingencies: that is, if form was the relevant dimension they continued to respond to that dimension on non-reinforced trials. "Non-conceptual" subjects did not. Using adult college students, Lowenkron found that the ID shift was performed more rapidly than the ED shift. It should be noted that subjects could change classification from 'conceptual' to 'non-conceptual' and vice versa from the acquisition phase to the transfer phase. Therefore the shift performance was evaluated as a function of post-shift solution mode. The ID shift was performed with fewer errors by 'consistent' than 
41.

'inconsistent' subjects, but performance did not differ as a function of solution mode in the ED shift. Lowenkron (1975) concluded that "the superior performance of consistent subjects in the ID relative to the ED shift gave support to a mediational interpretation of their behaviour" (p.453). Consistent subjects, he argued, learned to attend to the relevant dimension during acquisition allowing consistent behaviour on outcome trials and positive transfer to the ID shift. Inconsistent subjects, on the other hand, learned relatively specific associations between some of the stimuli and the correct responses, and therefore the ID-ED difference is attenuated. Before such evidence can be accepted unequivocally, the classification of subjects into 'consistent' and 'inconsistent' responders would require re-examination.

\section{Age effects.}

As part of a study to see whether age differences affected mediational processes, Shanab and Yasin (1979) included a group of young adults. Using a simultaneous ID-ED comparison Shanab and Yasin found college students executed the ID shift (12.25 trials to criterion) significantly faster than the ED shift (20.31 trials to criterion). Nehrke's (1973) data supported this finding. The ID shift (young adu1t) group produced a mean of 2.66 errors to criterion which did not differ significantly from original training, while the ED shift (young adult) group produced a mean of 4.12 errors which indicated significant negative mediational transfer from original training. 
Summary of Empirical Results

In summary, for experiments with human adults in the ID-ED shift comparison, the consistent finding is that in the post-shift phase, the ID shift is performed more accurately (in terms of errors to criterion) or faster (in terms of trials to criterion) than the ED shift over a range of conditions (eg. Isaacs \& Duncan, 1962: Nehrke, 1967: Shanab \& Yasin, 1979). This confirms the general finding for college students (Wolff, 1967) and children (Esposito, 1975a).

The extent of the ID-ED shift has been shown to be consistently affected by two main factors:-

i) Dimensional salience. The ID-ED shift difference may be sharply attenuated when a subject is being transferred to a high salience dimension relevant in the post-shift phase relative to being transferred to a low salience dimension (Esposito, 1973: Harrow, 1964: Ozioko \& May, 1977).

Although this was not the case for Esposito's (1975b) criterion-trained groups, the high salience groups consistently performed the ID and ED shifts faster than the low salience groups.

ii) Variability of the irrelevant dimension. The ID-ED shift difference may be attenuated by varying the irrelevant 
dimension between trials compared to within trials (Ozioko \& May, 1977). This is consistent with Esposito's (1975a) summary of studies comparing the variability of the irrelevant dimension in a simultaneous discrimination.

The ID-ED shift difference has been generated using a range of stimulus dimensions (Kennedy \& Gersten, 1976: LeBow \& Tritt, 1971: Slamecka, 1968). Interactions between the stimuli used and the type of shift can occur (Esposito, 1975b: Uh1, 1966).

ATTENTION AS TWO-DIMENSIONAL STIMULUS CONTROL

Traditionally, the difference in performance on the ID and ED shift is measured in terms of trials or errors to a pre-determined criterion. Such differences in learning speed or accuracy have been used to make inferences about the degree of control exerted by the relevant and irrelevant stimulus dimensions of the original discrimination and have been accounted for in terms of attention processes (eg. Esposito, 1975b: Harrow, 1964: LeBow \& Tritt, 1971: 0zioko \& May, 1977). For example, Kemler and Shepp (1971) compared trial-tolast-error data over six different shift types to test their hypotheses about changes in dimensional control during transfer. From significant differences in the TLE over the various shift types they concluded that their results supported the assumption that "both learned dimensional relevance and irrelevance contribute to performance in the transfer task" (p.126). Typically, however, they did not investigate the nature of degree of change in dimensional control and failure to trace such development in the transitional 
stages of transfer is prevalent throughout the literature on attention in discrimination learning. An exception is sub-problem analysis (eg. Tighe, Glick \& Cole, 1971) where discrimination shift data is analysed to examine a subject's response on each trial of a problem.

In most cases, therefore, the observation of an ID-ED difference, while establishing dimensional learning, does not directly reveal the specific source(s) of dimensional transfer. In order to assess this transitional stage more directly, a new approach is taken towards the transfer of attentional responses in adult human discrimination learning.

Ray (1972) argues that attention is an unstable phenomenon not well suited to the experimental design appropriate for stable phenomena. She believes that research in this area requires more emphasis on functional analyses that follow stimulus-response units through periods of transition. Functional analyses are well suited to examining the attention which Ray (1972) sees as a "critical relation between the environment and behaviour" (p.294).

The precedent for defining attention as a controlling relation was set by Skinner (1953) who saw attention as the relation between a response and a discriminative stimulus. Skinner's criterion for attention is whether or not a stimulus is exerting any effect on a particular behaviour. If the behaviour is a function of the stimulus, the observer is said to be attending to that stimulus. 
45.

Stimulus control serves a descriptive function and does not have explanatory or theoretical properties. Definitions of stimulus control are operational. Mackintosh (1977) has defined stimulus control as a term that has come "to be used as a convenient shorthand expression describing....... an observed relationship between changes in external stimuli and changes in recorded behaviour". This control is readily and conveniently assessed with the procedure that yields a generalisation gradient, a post-discrimination generalisation test.

Honig (1970) has suggested that attentional effects can be identified as modulations of stimulus control which take the form of general differences in the slopes of generalisation gradients. Attention arose as a theoretical issue in this context after it became clear that some stimulus dimensions yielded flatter gradients than others. The ID shift and the ED shift each involve two discrimination tasks and therefore seem ideally suited to the aforementioned analyses. Specifically, in discrimination learning, different consequences of behaviour are predicted by two or more stimulus values. One of these values may set the occasion for a given behaviour and absence of that stimulus value (or presence of another value) may occasion low probability of that behaviour. In that case a response differential or discrimination obtains and the behaviour is said to be under stimulus control. If the behaviour changes systematically when the values of the stimulus are varied along a dimension (as in a generalisation test) dimensional stimulus control is evident. In particular, if control by stimuli 
along one dimension is much greater than control along other dimensions, the subject is said to have attended to that dimension. In this sense attention is equivalent to the empirical demonstration of dimensional stimulus control.

Generalisation gradients have been used extensively to assess responding along specific dimensions following discrimination training in humans along one dimension (Doll \& Thomas, 1967: Howard, 1979: Newlin, Rodgers \& Thomas, 1979: Thomas, Svinicki \& Vogt, 1973: Thomas \& Vogt, 1983: White \& Thomas, 1979) and in humans and pigeons along two dimensions (Blough, 1972: Chase \& Heinemann, 1972: Johnson, 1970: Purtle \& Newman, 1969). Such tests involve the presentation of both training stimuli and other stimuli along one or more dimensions in a random fashion, usually without feedback to the subject.

Generalisation tests have several advantages over the other methods used in the ID-ED shift paradigm so far. Firstly, the subject is exposed to the entire dimension (s) during testing. Usually in the ID-ED shift comparison subjects are exposed to only four values of any one dimension and they do not have the opportunity to respond to those dimensions as a whole. The post-discrimination generalisation test provides, therefore, a better opportunity to discover the subject's response to the dimension which may not agree with the experimenter's classification (Ray, 1972).

Secondly, these tests are carried out in extinction. It is 
less likely that the testing procedure, which sets out to measure the extent of dimensional control, actually modifies control; it allows the subject's responses during testing to be relatively uncontaminated.

Thirdly, generalisation tests have the advantage that trends and failures in responding become apparent very quickly. Testing can begin at any point in time allowing assessment of control by dimensions to take place before, during and after learning to criterion in either Phase-1 or Phase-2. It does not restrict comparisons to post-Phase-1 and Phase-2 terminal performance. Responding can therefore be evaluated over trials (cf. subproblem analysis). There is an increased likelihood that rapid transitions in stimulus control would be detected (Ray \& Sidman, 1970).

Because all subjects have undergone the same testing procedure and have been exposed to the same set of stimuli, the results of the experiment lend themselves to different kinds of analyses.

\section{The Present Experiments}

It is the aim of the following series of experiments to systematically investigate some factors that affect the magnitude of the ID-ED shift difference, and to use a framework that permits a closer examination of the degree of control by stimulus dimensions. Two-process theories (Lovejoy, 1968: Sutherland \& Mackintosh, 1971: Zeaman \& House, 1963) predict an ID shift superiority over the ED shift when two dimensions are simultaneously presented in a 
48.

discrimination task. The present research extends this prediction to successive discrimination (cf. Evans \& Hogg, 1976: Hogg \& Evans, 1975, 1978).

Experiment 1 compared data from post-discrimination generalisation tests to the trials-to-criterion data and assessed the relative dominance of the two dimension employed here (1ine length and line orientation) by counterbalancing over shift conditions. In Experiment 2 the relative stimulus values on the relevant dimension in Phase-1 were reversed in the Intradimensional shift in Phase-2 to show that any ID-ED differences could not be accounted for in terms of stimulus generalisation, but rather, involved attending to dimensions. In Experiment 3, development of stimulus control along both length and orientation dimensions during Phase-2 of the ID and ED shifts was investigated by including different numbers of Phase-2 training trials before generalisation testing. Experiment 3 thus demonstrated the gradual development of control by the previously irrelevant dimension in the ED shift, concurrent with a gradual loss of control by the previously relevant dimension.

Experiments 4 to 6 focused on the components of the ED shift. Experiment 4 attempted to differentiate between the two processes of learned relevance and learned irrelevance which are confounded in the ID-ED shift comparison. The effects of prior extradimensional discrimination training were compared to those of non-differential discrimination training by using a pseudo-discrimination paradigm. 
49.

Experiment 5 examined whether the variability of the irrelevant dimension is important in determing the size of the ID-ED shift (cf. Esposito, 1975). In Experiment 5 there were two stimulus values on the irrelevant dimension or one value (constant irrelevant), in both Phase-1 and Phase-2 discriminations.

Experiment 6 repeated the conditions of Experiment 5 but with only one dimension varied in the generalisation test, in order to examine the contribution of masking control in the ED shift by the relevant dimension of the Phase-1 discrimination. 
EXPERIMENTS 


\section{EXPERIMENT 1}

Most previous studies of the ID-ED shift have involved a transfer paradigm in which discrimination training in Phase-1 is followed by discrimination training in Phase- 2 where the training values are on the dimension that was relevant (ID) or irrelevant (ED) in Phase-1. Performance on the transfer task has typically been assessed in terms of trials to a given criterion (trials correct) or errors to criterion. Inferences about stimulus dimensions that have been exerting control over discriminative performance have therefore been drawn from between group comparisons of discriminative performance rather than based directly on the results of empirical generalisation gradients.

The aim of Experiment 1 was to ask whether the relevant stimulus dimensions in the Phase-1 and Phase-2 tasks of an ID-ED shift did indeed exert dimensional stimulus control over performance, and whether that control was consistent with the typical ID-ED shift effect (cf. Hogg \& Evans, 1975, 1978). Different groups of subjects were given a generalisation test in which all possible combinations of a range of stimulus values on two dimensions were presented. The test was administered after Phase-1 training or after Phase-2 training for ID and ED transfer groups.

The stimulus dimensions used in the present experiments were the length and orientation of a single line. The design of the experiment (Table 1) counterbalanced ID and ED shifts over the length and orientation dimensions in order to establish the possible 
51.

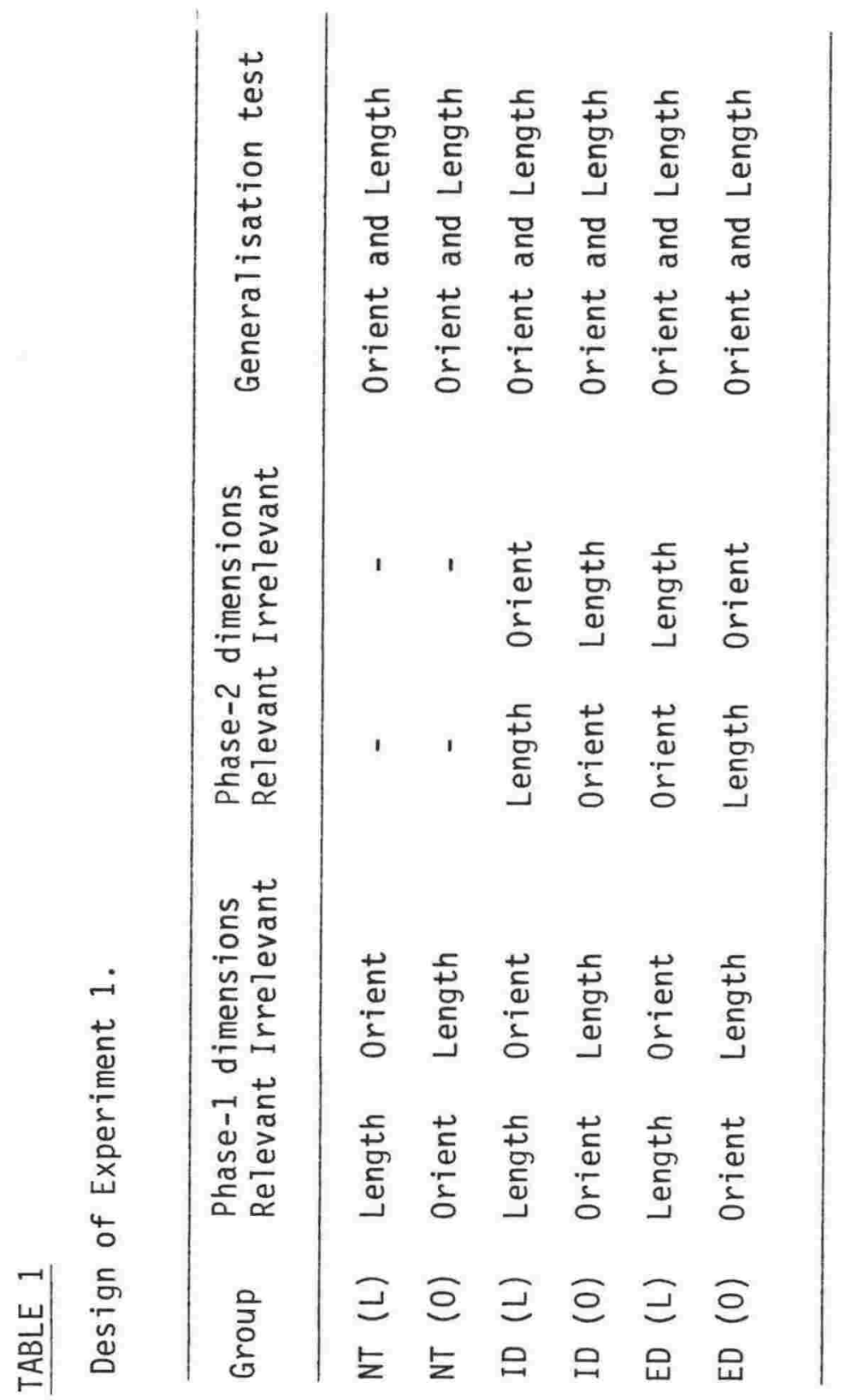


contribution of stimulus dominance to the ID-ED shift. Honig (1969) states that it is important when examining the transfer of stimulus control to systematically counterbalance the experimental design to demonstrate that any effects are attentional in nature, rather than specific to a particular relationship between stimulus dimensions.

A dominance relationship between dimensions has accounted for discrepancies in obtaining the ID-ED performance difference in previous studies (Esposito, 1973: Harrow, 1964: Ozioko \& May, 1977).

In Experiment 1, ID groups, ED groups and groups trained only in the Phase-1 task (NT groups), were trained with length or orientation as the relevant Phase-1 dimensions. As shown on Table 1, whether length or orientation was the relevant dimension in Phase-2 training was determined by the shift (ID or ED). In the generalisation test, seven values of orientation were combined with seven values of length in order to assess conjoint control by both dimensions.

METHOD

Subjects were 48 undergraduates who were randomly allocated between six groups with the constraint that each group comprised 5 males and 3 females. One subject in the ED (L) group was replaced because of failure to reach the performance criterion within the pre-set limit of 70 Phase-2 trials.

Apparatus. The experiment was conducted in a $4^{\prime}$ by $5^{\prime}$ white walled 
room. The experimenter was separated from the subject by a rigid plastic screen $(400 \mathrm{~mm} \times 500 \mathrm{~mm})$ mounted on a small table. The screen had a central rectangular hole $(100 \mathrm{~mm} \times 60 \mathrm{~mm})$ where stimulus cards were displayed. The stimuli were constructed from $3 \mathrm{~mm}$ wide letraset lines in the centre of white cards $(126 \mathrm{~mm} \times 75 \mathrm{~mm})$. There were 49 different stimuli. These comprised all combinations of line lengths $(8 \mathrm{~mm}, 10 \mathrm{~mm}, 12 \mathrm{~mm}, 14 \mathrm{~mm}, 16 \mathrm{~mm}, 18 \mathrm{~mm}, 20 \mathrm{~mm})$ and 1 ine orientation $\left(75^{\circ}, 65^{\circ}, 55^{\circ}, 45^{\circ}, 35^{\circ}, 25^{\circ}, 15^{\circ}\right.$ from vertical). Verbal responses from each subject were recorded by the experimenter. Viewing distance was $60 \mathrm{~cm}$.

Procedure. Each subject was seated in front of the screen and was instructed as follows:

\footnotetext{
"This is an experiment in perception. There are a number of of trials and on each trial I will show you a line for three seconds. According to a rule there will be a 'yes' line or a 'no' line. I would like you to reply according to the following scale. (Subjects were given a six point scale to keep for the duration of the experimental session). For example, if you are very certain that the line you see is a 'yes' line you should say ' 6 ', fairly certain ' 5 ', not very certain ' 4 '. If you are very certain the line you see is a 'no' line you should say '1', fairly certain '2', not very certain ' 3 '. Obviously your response on the very first trial will be a guess. Your aim will be to identify a 'yes' and a 'no' line correctly. On some trials I will tell you whether or not you are correct, while on others I would like you to continue without my assistance. Remember that on every trial you should give me a number according to how certain you are that the line is a 'yes' or a 'no' line. Do you have any questions?"
}

Phase-1 discrimination training. Phase-1 discrimination training was identical for all subjects, except that for 24 subjects length was the relevant dimension, and for 24 subjects, orientation was the relevant 
dimension. Training stimuli used were lines of $8 \mathrm{~mm}$ or $20 \mathrm{~mm}$ long, oriented $15^{\circ}$ or $75^{\circ}$ from vertical. The four training stimuli were presented equally often and one at a time for 3 seconds on each trial, in a random order over 24 training trials. The intertrial interval approximated 5 seconds.

The subject's rating response to each 1 ine was followed by 'correct' or 'incorrect' feedback from the experimenter according to the following rule. For the length-relevant groups, NT (L), ID (L), and $\operatorname{ED}(\mathrm{L})$, lines to which 'yes' ratings were required were $20 \mathrm{~mm}$ lines oriented $15^{\circ}$ or $75^{\circ}$, and lines which required a 'no' rating were $8 \mathrm{~mm}$ lines oriented $15^{\circ}$ or $75^{\circ}$. That is, the length, but not the orientation values were relevant to the discrimination. For the orientation-relevant groups, NT (0), ID (0) and ED (0), 'yes' lines were $75^{\circ}$ orientation either $8 \mathrm{~mm}$ or $20 \mathrm{~mm}$ long, and 'no' 1 ines were $15^{\circ}$ orientations either $8 \mathrm{~mm}$ or $20 \mathrm{~mm}$ long. That is for these groups, orientations, but not length were relevant to the discriminations.

Phase-2 discrimination training. Trials of Phase-2 discrimination continued from Phase-1 training without a break in the procedure. The main procedural requirements were the same except that different stimulus values and different response rules were introduced. The two NT (No Transfer) groups were not given Phase-2 trials, but proceeded directly to the generalisation test. The ID and ED groups saw four new lines over as many trials as were necessary for each subject to attain a criterion of 10 correct responses over consecutive trials. The experimenter's 'correct' or 'incorrect' feedback on each 
55.

trial was determined by the following rules. For the length relevant groups, 'yes' lines were $18 \mathrm{~mm}$ long oriented $25^{\circ}$ or $65^{\circ}$ and 'no' lines were $10 \mathrm{~mm}$ long oriented $25^{\circ}$ or $65^{\circ}$ (ie. the ID (L) group and the ED (0) group). For the orientation relevant groups, 'yes' lines were $65^{\circ}$ Iines either $10 \mathrm{~mm}$ or $18 \mathrm{~mm}$ long and 'no' 1 ines were $25^{\circ}$ lines either $10 \mathrm{~mm}$ or $18 \mathrm{~mm}$ long (ie. the ID (0) group and the ED (L) group).

Generalisation testing. A generalisation test was administered directly following Phase-1 training for the NT groups or directly following Phase-2 training for the ID and ED groups without a break in the procedure. Stimulus presentations followed the same procedure as in training, except that there was no feedback from the experimenter. The test comprised presentations of 49 unique stimuli combining 7 length values with 7 orientation values. The random order of presentation of the 49 lines over the first 49 trials was reversed for a second block of 49 trials. Presentation orders were constrained by not allowing a given stimulus value on either dimension to occur on consecutive trials. The test was continued without feedback from the experimenter.

RESULTS

Phase-2 discrimination. Table 2 gives the mean triais to criterion required by the ID and ED groups in the Phase-2 discrimination. The ID-ED shift effect reported in previous studies was also clearly evident in the present experiment. The ED groups required twice as many trials as ID groups to reach criterion, $F(1,28)=18, p<.001$. 
56.

\section{TABLE 2}

Mean trials to criterion for ID and

ED groups in Phase-2 discrimination

of Experiment 1.

\begin{tabular}{lcr}
\hline Group & Mean & SD \\
\hline ID (L) & 10.25 & 0.71 \\
ED (L) & 24.5 & 12.46 \\
& & \\
ID (0) & 10.13 & 0.35 \\
ED (0) & 24.38 & 8.3 \\
\hline
\end{tabular}


The ID-ED shift effect was independent of the stimulus dimension in that the type of Phase-1 discrimination did not affect trials to criterion, $F<1$, and there was no interaction between groups and dimension, $F<1$. In addition, correct responses are plotted against number of trials for ID and ED groups in Figure 4. This shows the rate of acquisition for the two tasks is faster for both ID groups than both ED groups.

Generalisation. Mean response ratings to each stimulus value in the generalisation test were derived for each group by averaging ratings over trials and over subjects within groups. Gradients for each group were thus based on a total of 16 observations for each of the 49 stimulus values. The gradients were plotted on isometricorthographic co-ordinates to show variations in mean ratings along both orientation and length dimensions (cf. Johnson: 1970).

Figure 5 shows gradients obtained after Phase-1 training for the NT (1) and NT (0) groups. These gradients demonstrate that the relevant dimensions in Phase-1 discrimination had gained dimensional stimulus control in that mean ratings for the NT (L) group varied systematically with length, but not with orientation (the irrelevant dimension). The mean ratings for the NT (0) group varied systematically with orientation but not with length (the irrelevant dimension). The mean ratings in Figure 5 had maxima at the stimulus value, $20 \mathrm{~mm}$ or $75^{\circ}$, requiring 'yes' ratings in prior discrimination training. Functions were flat along the irrelevant dimension, indicating absence of control by that dimension. 

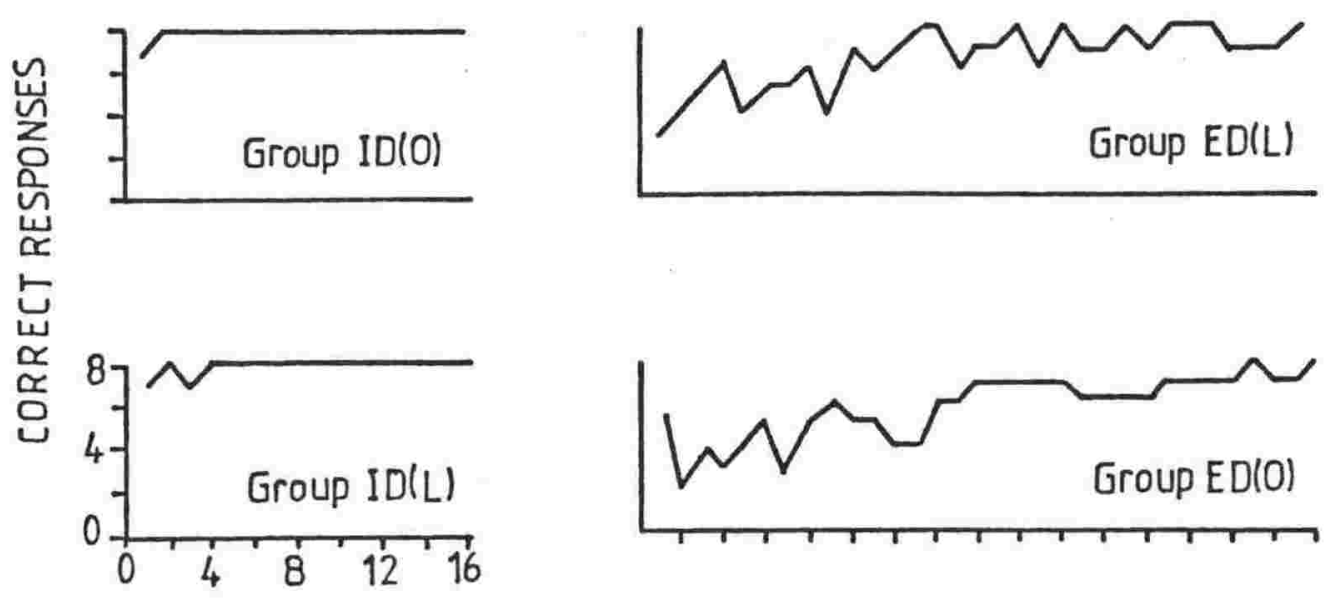

TRIALS

Figure 4: Number of correct responses per group as a function of number of trials of ID (ID (0), ID (L)) or ED (ED (0), ED (L)) Phase-2 discrimination training. 
59.

(i)

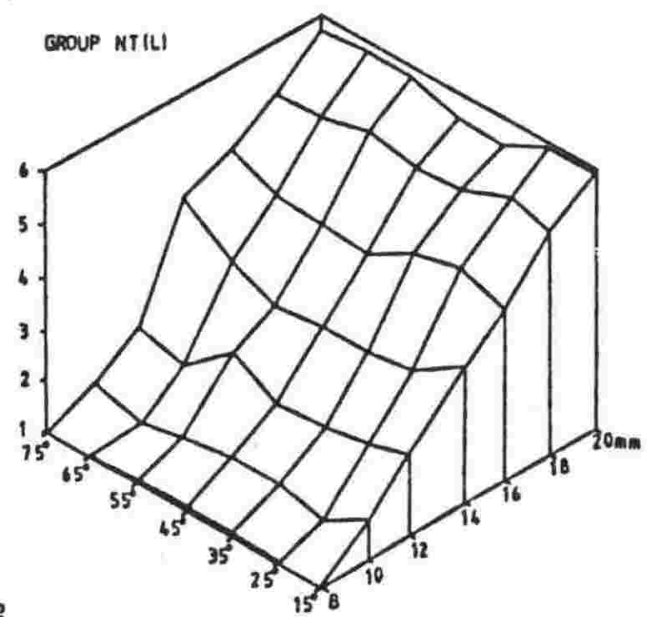

(ii)

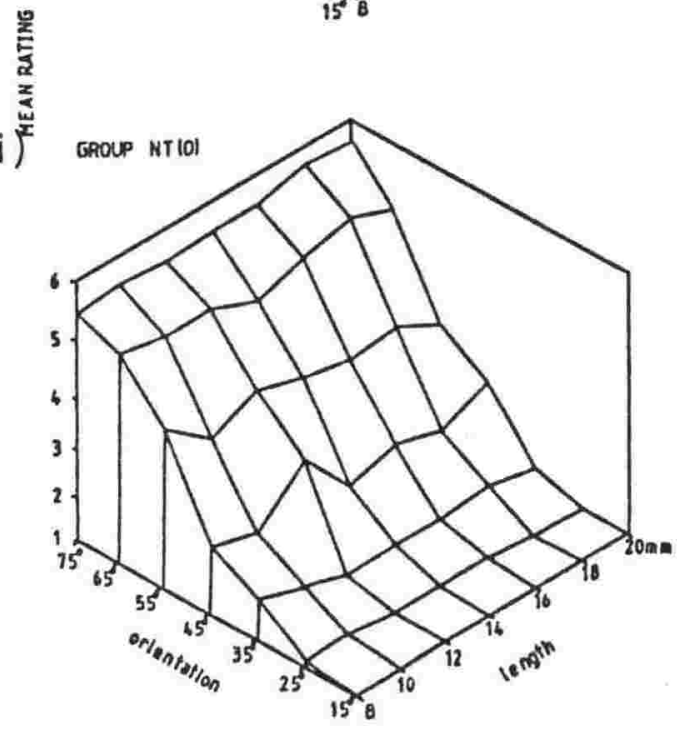

Figure 5: Post Phase-1 two-dimensional generalisation gradients following either (i) 24 trials of a length discrimination or (ii) 24 trials of an orientation discrimination showing mean response ratings as a function of length and orientation dimensions. 
60.

The gradients were unaffected by whether the relevant dimension was orientation or length in that there was no effect of group, $\mathrm{F}<1$, or interaction with groups, in an analysis of variance in which factors were group NT (L) versus NT $(0)$, relevant dimension (7 values) and irrelevant dimension ( 7 values). Separate analyses of variance for NT (L) and NT (0) confirmed control by the relevant dimensions length $(F(6,42)=107.07, p<.001)$ and orientation $(F(6,42)=63.45 \mathrm{p}<.001)$, and absence of control by the irrelevant dimensions orientation $(F(6,42)=1.80$, n.s.) and length $(F(6,45)=$ 1.47 , n.s.) in NT (L) and NT (0) respectively.

Figure 6 shows that gradients for individual subjects within a group were consistent with group mean gradients. In Figure 6 , mean ratings are plotted for orientation, averaged over all lengths, and for length averaged over all orientations. For each subject in the two groups, gradients along the relevant dimension were steep and gradients along the irrelevant dimension were flat.

Gradients obtained following Phase-2 discrimination training for the ID and ED groups are shown in Figure 7. A11 gradients are sharp along the dimension that was relevant in Phase-2 discrimination training, and flat along the dimension that was irrelevant in Phase-2 discrimination. Results of analyses of variance for the effect of the relevant dimension in each group is shown in Table 3 . The F values for all irrelevant dimensions and interactions were nonsignificant. 
61.

\section{TABLE 3}

Summary of analyses of variance to show the effects of the relevant and irrelevant dimensions in the four experimental groups in Experiment 1, following Phase-2 (criterion) discrimination training.

\begin{tabular}{|c|c|c|c|c|c|c|}
\hline CONDITION & DIMENSION & DF & MS & $\mathrm{F}$ & & \\
\hline ID $(L)$ & $\begin{array}{l}\text { Length }(R E L) \\
\text { Orient }(I R R) \\
L \times 0\end{array}$ & $\begin{array}{c}6,42 \\
6,42 \\
36,252\end{array}$ & $\begin{array}{r}137.4 \\
0.7 \\
0.2\end{array}$ & $\begin{array}{c}33.0 \\
2.0 \\
<1\end{array}$ & $\mathrm{p}$ & $\begin{array}{l}<.001 \\
\text { n.s. } \\
\text { n.s. }\end{array}$ \\
\hline ID $(0)$ & $\begin{array}{l}\text { Orient (REL) } \\
\text { Length }(I R R) \\
L \times 0\end{array}$ & $\begin{array}{c}6,42 \\
6,42 \\
36,252\end{array}$ & $\begin{array}{r}217.5 \\
0.2 \\
0.2\end{array}$ & $\begin{array}{l}65.8 \\
<1 \\
<1\end{array}$ & $p$ & $\begin{array}{l}<.001 \\
\text { n.s. } \\
\text { n.s. }\end{array}$ \\
\hline$E D(L)$ & $\begin{array}{l}\text { Orient }(R E L) \\
\text { Length }(I R R) \\
L \times 0\end{array}$ & $\begin{array}{c}6,42 \\
6,42 \\
36,252\end{array}$ & $\begin{array}{r}212.7 \\
0.4 \\
0.2\end{array}$ & $\begin{array}{c}65.2 \\
1.1 \\
<1\end{array}$ & $\mathrm{p}$ & $\begin{array}{l}<.001 \\
\text { n.s. } \\
\text { n.s. }\end{array}$ \\
\hline ED (0) & $\begin{array}{l}\text { Length (REL) } \\
\text { Orient (IRR) } \\
L \times 0\end{array}$ & $\begin{array}{c}6,42 \\
6,42 \\
36,252\end{array}$ & $\begin{array}{r}183.0 \\
0.7 \\
0.4\end{array}$ & $\begin{array}{r}42.9 \\
1.0 \\
<1\end{array}$ & $\mathrm{p}$ & $\begin{array}{l}<.001 \\
\text { n.s. } \\
\text { n.s. }\end{array}$ \\
\hline
\end{tabular}


(i)
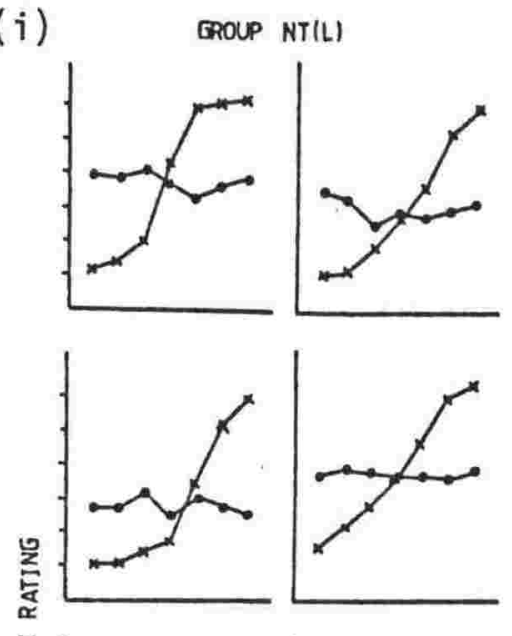

永
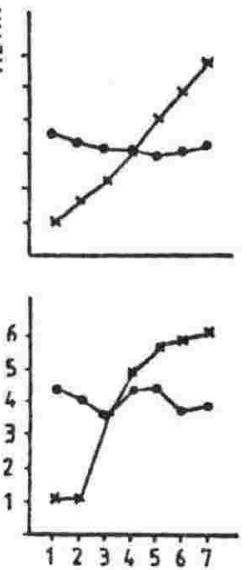

(ii)
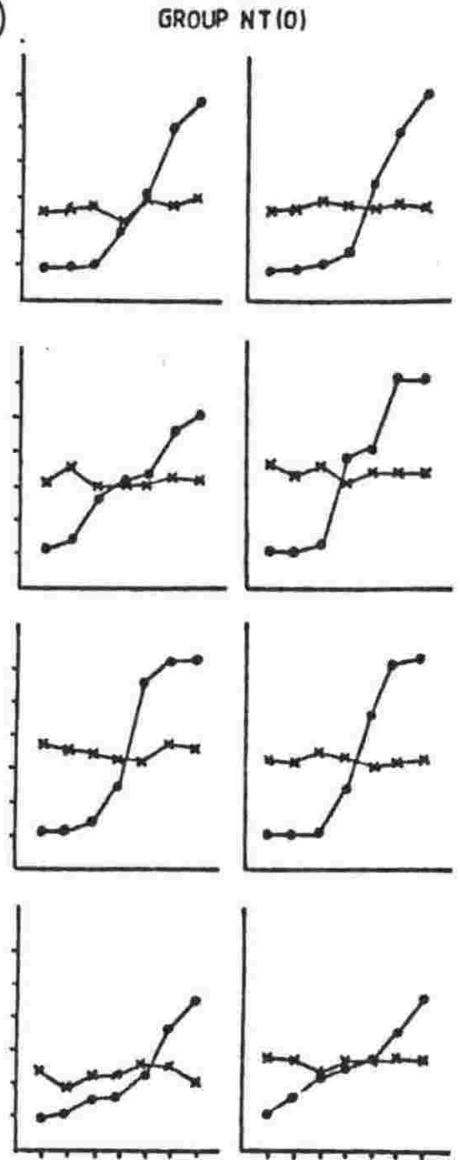

ORDINAL LENGTH (x) OR ORIENTATION (0) VALUE

Figure 6: Post Phase-1 general isation gradients following either (i) 24 trials of length discrimination with Stimulus 7 as 'yes' and Stimulus 1 as 'no' or (ii) 24 trials of orientation discrimination with Stimulus 7 as 'yes' and Stimulus 1 as 'no', showing mean response ratings for individual subjects as a function of either length $(x)$ or orientation $(\bullet)$. 
63.
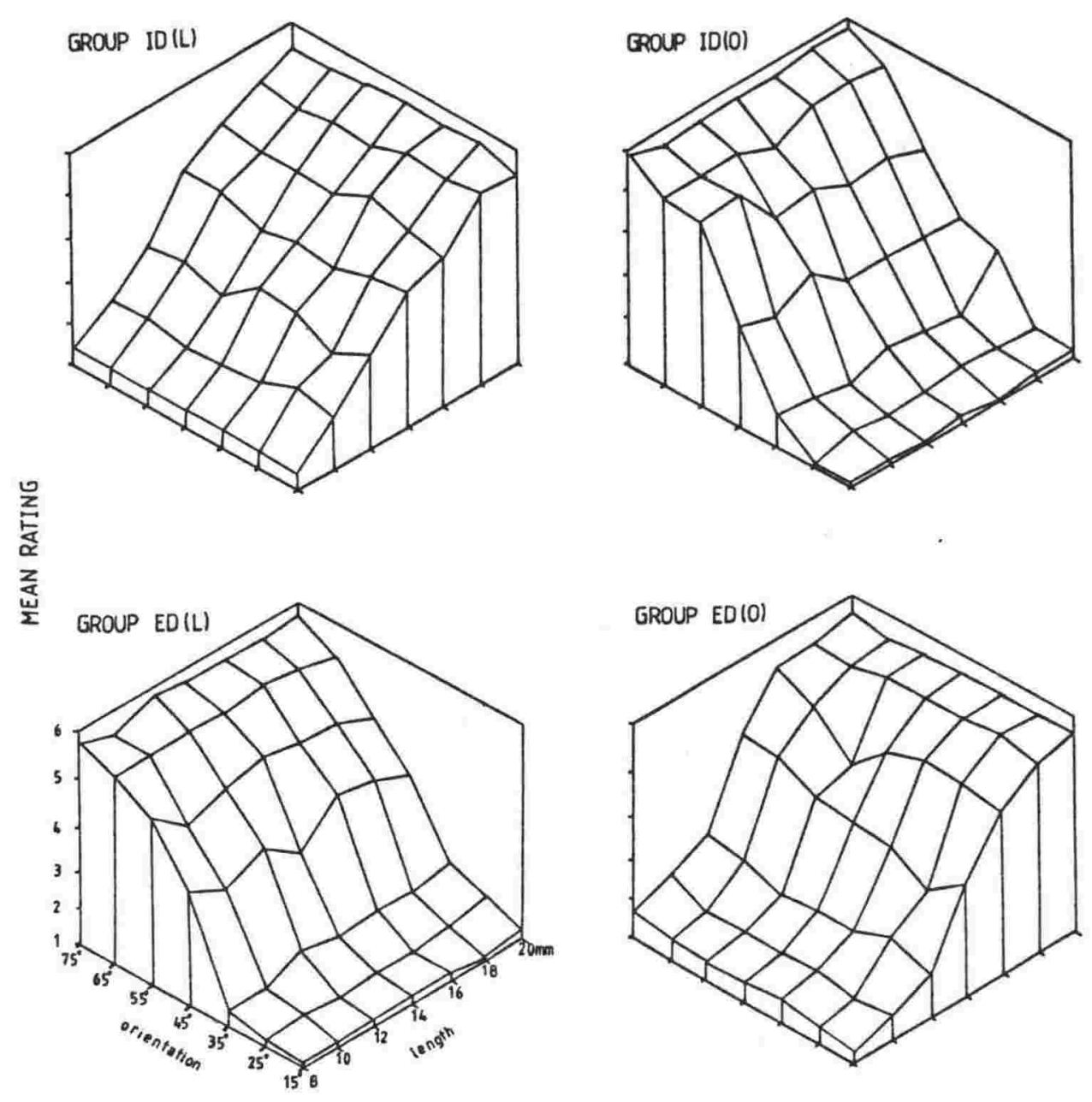

Figure 7: Post Phase-2 two-dimensional generalisation gradients following criterion performance on either the length discrimination [ID (L) and ED (O)] or the orientation dimension [ID (O) and ED (L)] showing group mean response ratings as a function of length and orientation dimensions. 
64.

The gradients following Phase-2 tend to be more ogival in form than the Phase-1 gradients, owing to the Phase-2 training stimuli lying at positions 2 and 6 on the training dimension rather than at positions 1 and 7 . Otherwise the post-Phase- 2 gradients have the same form as the post-Phase-1 gradients. There is no difference in the gradients as a function of the dimension that was relevant in Phase-1. The intervals for length and orientation values are functionally equivalent and there is no evidence for dimensional dominance.

The gradients in Figure 7 exhibit the main characteristics of the ID-ED shift. For the ID gradients, the dimension that was relevant in Phase-1 and remained relevant in Phase-2 retains dimensional stimulus control. For the ED gradients, the dimension that was relevant in Phase-1 has lost control following Phase-2 training, whereas the previously irrelevant dimension has gained dimensional stimulus control. The degree of control exerted by the relevant dimensions for the ID and ED groups is similar, owing to transfer having been continued until a criterion level of performance was reached.

\section{DISCUSSION}

The present experiment demonstrated the ID-ED shift in terms of trials to criterion in a successive discrimination. The performance differential was comparable to those found in previous studies of simultaneous ID-ED comparisons. Shanab and Yasin (1979) included a group of young adults in their study of ID-ED differences. 
Using a simultaneous design and an identical performance criterion (10 consecutive trials correct), they found a mean trial to criterion of 12.25 (ID) and 20.31 (ED). This is almost the same as the mean trials to criterion for the ID (10.18) and ED (24.44) groups in the present experiment. The trials to criterion data establish that the ID is performed significantly faster than the ED. The post-discrimination generalisation gradients allowed a comparison of dimensional stimulus control both before and after transfer. They provide direct evidence of attention to a specific dimension and demonstrate how control is related to specific cues on the dimension. That is, control by the same relevant dimension is evident following both Phase-1 and Phase-2 discrimination training in the ID groups, whereas in the ED groups control by the relevant dimension following Phase-1 is lost after Phase-2 when that dimension becomes irrelevant, and control by the new relevant dimension is exhibited.

The present experiment also demonstrated that the extent of the ID-ED shift and also the extent of dimensional stimulus control was unaffected by the specific nature of the dimensions used, length or orientation. That is, there was no evidence for dimensional dominance. Harrow (1964) found that the ID-ED shift can be affected by the direction of change between a relatively dominant and nondominant dimension. For example, for groups that shifted from colour to shape, the ED group performed the transfer task slower than the ID group (38 versus 4 trials to criterion). For the shape to colour group, this difference in performance was attenuated 
( 6 versus 2 trials to criterion). Fortunately, the present result is not confounded by the presence of dimensional dominance and the length and orientation dimensions used here can be regarded as functionally equivalent. 
67.

\section{EXPERIMENT 2}

The main theoretical significance of the ID-ED shift is that it demonstrates attention to dimensions independently of attention to specific cues. In Experiment 1 it was shown that the relevant dimension did exert dimensional stimulus control in the pre-shift and post-shift discrimination tasks, that is, the relevant dimensions were being attended to and the irrelevant dimensions were not. It is possible, however, that dimensional control in the ID groups was mediated by cue-specific transfer because the discrimination between $20 \mathrm{~mm}$ versus $8 \mathrm{~mm}$ in Phase-1 was shifted to a discrimination between $18 \mathrm{~mm}$ versus $10 \mathrm{~mm}$ in Phase-2. That is, the faster transfer by the ID group could be attributable to stimulus generalisation from Phase-1 to Phase-2 stimuli.

Previous studies (LeBow \& Tritt, 1971: Shanab \& Yasin, 1979) have used qualitative dimensions such as form and words, and it is less likely that stimulus generalisation would have occurred in the ID shift. In Experiment 2, the possibility of cue-specific generalisation in the present procedure was examined by comparing ID and ED shifts conducted in Experiment 1 to ID and ED shifts involving a reversal of the specific stimulus values on the relevant dimensions. In the ID Reversal groups, a Phase-1 discrimination between $20 \mathrm{~mm}$ (yes) and $8 \mathrm{~mm}$ (no) was transferred to a Phase-2 discrimination between $10 \mathrm{~mm}$ (yes) and $18 \mathrm{~mm}(\mathrm{no})$. Only groups performing a line length discrimination were used in this experiment, as it was established in Experiment 1 that proceeding either from length or orientation or vice versa did not affect experimental 
68.

results.

METHOD

Subjects were 32 undergraduates. Four males and four females were assigned to each of four groups. Apparatus and the general procedural conditions were the same as those in Experiment 1. All subjects in all groups were first trained for 24 trials in Phase-1 under identical conditions, with length relevant and orientation irrelevant. In Phase-1 'yes' ratings were required for $20 \mathrm{~mm} 1$ ines oriented at $15^{\circ}$ or $75^{\circ}$ and 'no' ratings were required to $8 \mathrm{~mm}$ lines oriented at $15^{\circ}$ or $75^{\circ}$.

Following Phase-1, all subjects were given a generalisation test without a break in the procedure. The test was administered without feedback from the experimenter, and involved presentation of 49 lines comprising all possible combinations of seven lengths and seven orientations. The random order of presentation of the 491 ines was reversed for a second block of 49 trials.

The pre-shift generalisation test was immediately followed by Phase-2 discrimination training without a pause in the procedure. Responses were followed by 'correct' and 'incorrect' feedback from the experimenter and training continued until a criterion of 10 correct consecutive trials was attained. Phase-2 discrimination was immediately followed by a 98 trial generalisation test in which length and orientation varied conjointly identical to the preshift generalisation test. 
69.

The rule relating 'yes' and 'no' ratings to specific stimulus values was different for four groups and is given in Table 4. In two ID groups, length was the relevant dimension in Phase-2 and in two ED groups orientation was the relevant dimension in Phase-2. For the Reversal groups, training values were the reverse of those used in the 'non-reversal' groups (Table 4).

\section{RESULTS}

Table 5 gives the mean trials to criterion required by the ID and ED groups in the Phase-2 discrimination from Experiments 1 and 2. The ID-ED shift effect reported in Experiment 1 with ID (L) and ED (L) was clearly evident in the present experiment for the reversal ID (R) group and the pseudo-reversal ED (R) group, and it was of the same order. ED groups required twice as many trials to criterion as ID groups $F(1,28) 11.89, p<.001$. The ID-ED shift effect was independent of the values on the stimulus dimensions, in that non-reversal and reversal groups showed no difference in trials to criterion, $\mathrm{F}<1$, and there was no interaction between type of shift and reversal condition, $F<1$. In Figure 8 , correct responses are plotted against number of trials of Phase-2 training. This shows a much faster rate of acquisition of the Phase-2 task for ID and ID (R) groups compared to the ED and ED (R) groups.

Mean response ratings to each stimulus value in the generalisation test were derived for each group as in Experiment 1. Figure 9 (i) shows gradients obtained after Phase-1 training for the ED, ED (R), ID and ID (R) groups. These gradients demonstrate 


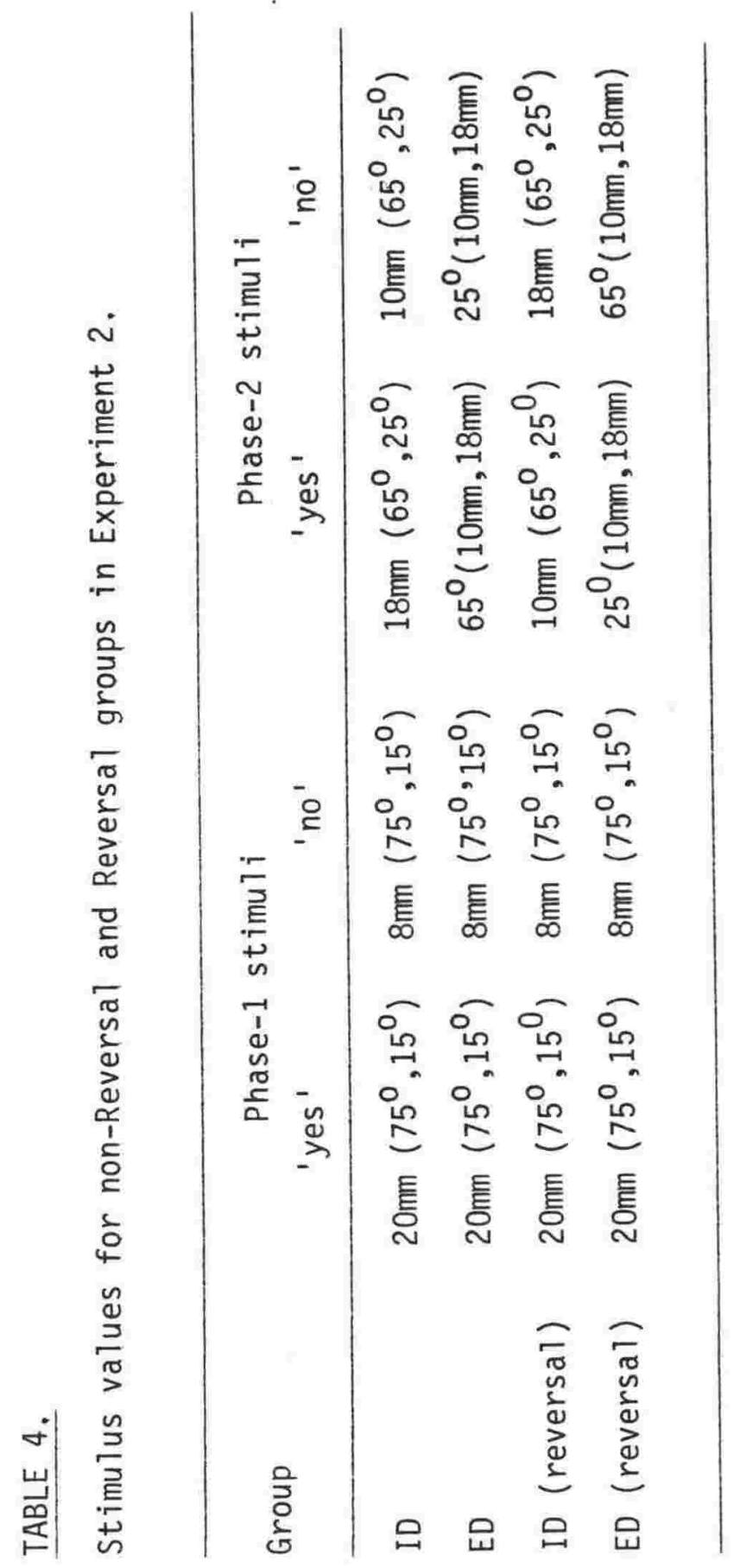




\section{TABLE 5}

Mean trials to criterion in the Phase-2 discrimination for the ID, ID (R), ED and ED (R) groups.

\begin{tabular}{llr} 
Group & Mean & SD \\
\hline ID & 10.25 & 0.71 \\
ID (reversa1) & 11.5 & 0.76 \\
ED & 24.5 & 12.46 \\
ED (reversa1) & 24.13 & 13.42
\end{tabular}


72.

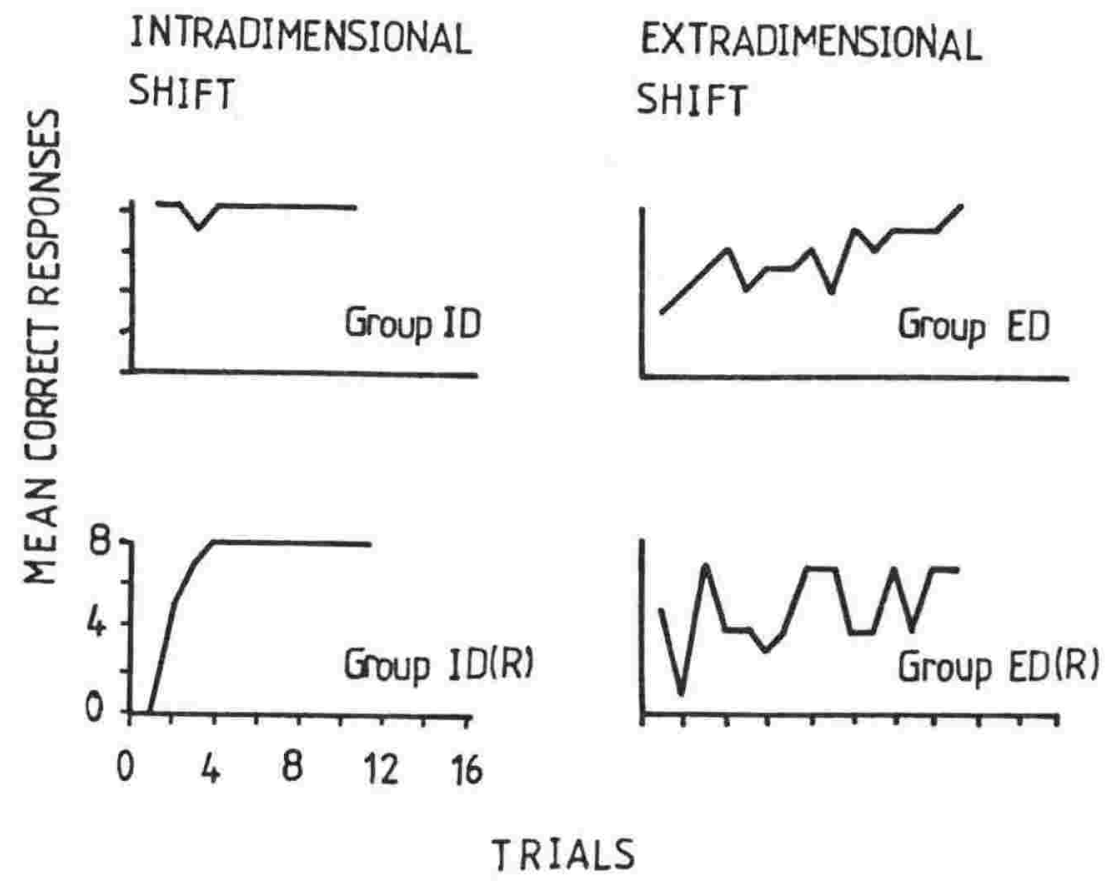

Figure 8: Number of correct responses per group as a function of the number of trials of either intradimensional [ID and ID (R)] or extradimensional [ED and ED (R)] Phase-2 discrimination trainina. 
(i) PRESHIEI
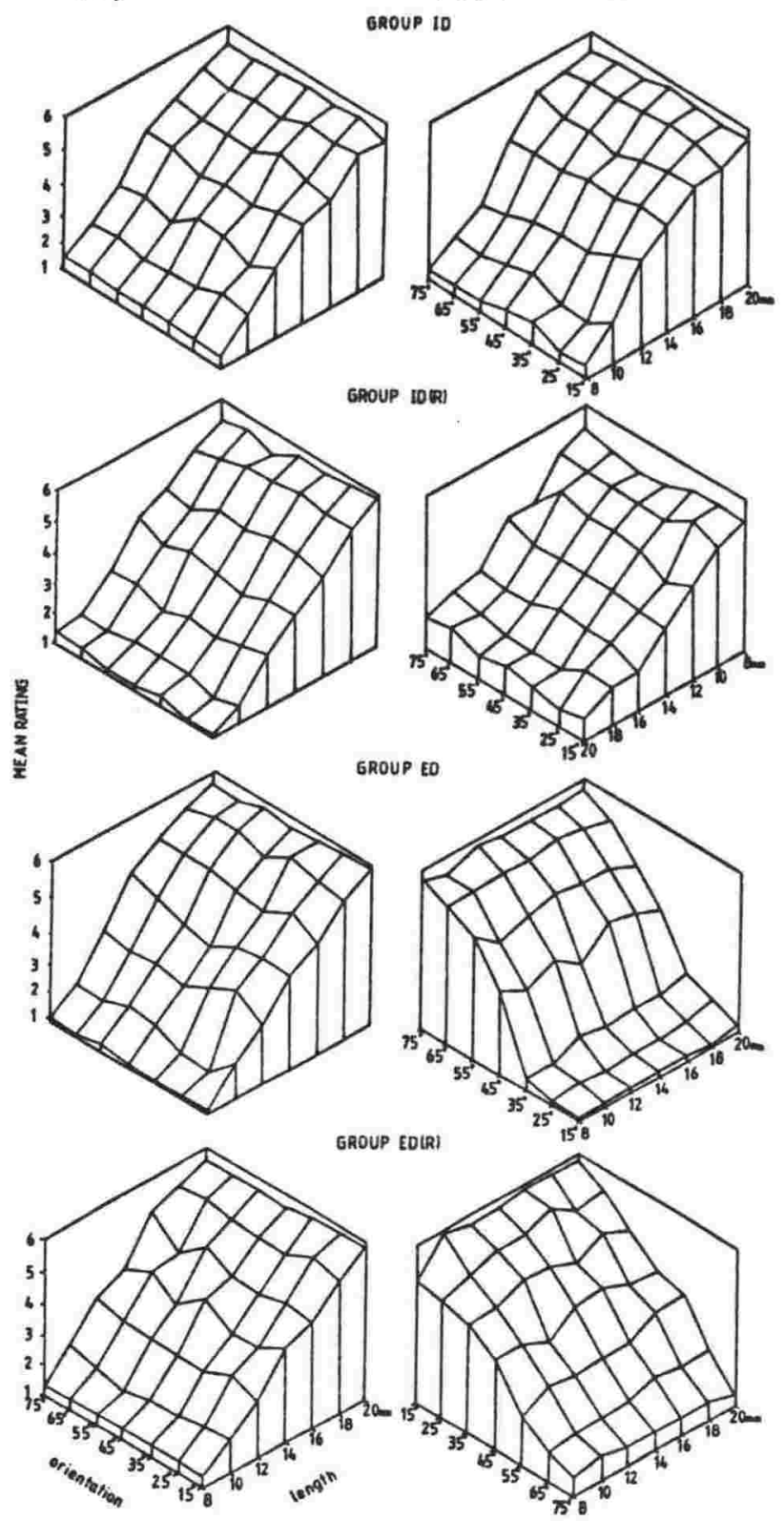

Figure 9: Two-dimensional generalisation gradients following either (i) 24 trials of Phase-1 line length discrimination or (ii) criterion performance in Phase-2, on either the length dimension [ID and ID (R)] or the orientation dimension $[E D$ and $E D(R)]$ showing group mean response ratings as a function of length and orientation dimensions. 
that line length, the relevant dimension in Phase-1 discrimination had gained dimensional stimulus control in that mean ratings for all groups varied sytematically with length, but not with orientation (the irrelevant dimension). The mean ratings in Figure 9 (i) had maxima at the stimulus value, $20 \mathrm{~mm}$, requiring 'yes' ratings in Phase-1 discrimination training. Functions were flat along the irrelevant dimension, indicating absence of control by that dimension. Results of analyses of variance for the four conditions are summarised in Table 6 and confirm these trends.

Figure 10 shows that gradients for individual subjects within a group were consistent with group mean gradients. For each subject in the four groups, gradients along the relevant length dimension were steep and gradients along the irrelevant orientation dimension were flat.

Gradients obtained following Phase-2 discrimination training for the ID, ED reversal and non-reversal groups are shown in Figure 9 (ii). All gradients are sharp along the dimension that was relevant in Phase-2 discrimination training, and flat along the dimension that was irrelevant in Phase-2 discrimination training. Table 6 shows the results of analyses of variance conducted for Phase-2 of each group and confirms the degree of control by the relevant dimension. All effects for Phase-2 irrelevant dimensions were non-significant $(F(6,42)<1)$. The gradients shown in Figure 9 (ii), exhibit the main characteristics of the ID-ED shifts as in Experiment 1. For the ID shift the dimension that was relevant 


\section{TABLE 6}

Summary of analyses of variance to show the effects of the relevant dimension in the eight experimental groups in Experiment 3 after both (i) Phase-1 and (ii) Phase-2 (criterion) discrimination learning.

CONDITION DIMENSION DF MS F

(i) PHASE-1

$\begin{array}{llllll}\text { ID } & \text { Length } & 6,42 & 163.0 & 50.1 & p<.001 \\ \text { ID (R) } & \text { Length } & 6,42 & 177.0 & 96.0 & p<.001 \\ \text { ED } & \text { Length } & 6,42 & 184.0 & 196.1 & p<.001 \\ \text { ED (R) } & \text { Length } & 6,42 & 152.0 & 198.31 & p<.001\end{array}$

(ii) PHASE-2

$\begin{array}{lrrrrr}\text { ID } & \text { Length } 7-1 & 6,42 & 137.0 & 33.0 & \mathrm{p}<.001 \\ \text { ID (R) } & \text { Length 1-7 } & 6,42 & 99.0 & 11.5 & \mathrm{p}<.001 \\ \text { ED } & \text { Orient 7-1 } & 6,42 & 213.0 & 65.2 & \mathrm{p}<.001 \\ \text { ED (R) } & \text { Orient 1-7 } & 6,42 & 139.0 & 90.2 & \mathrm{p}<.001\end{array}$




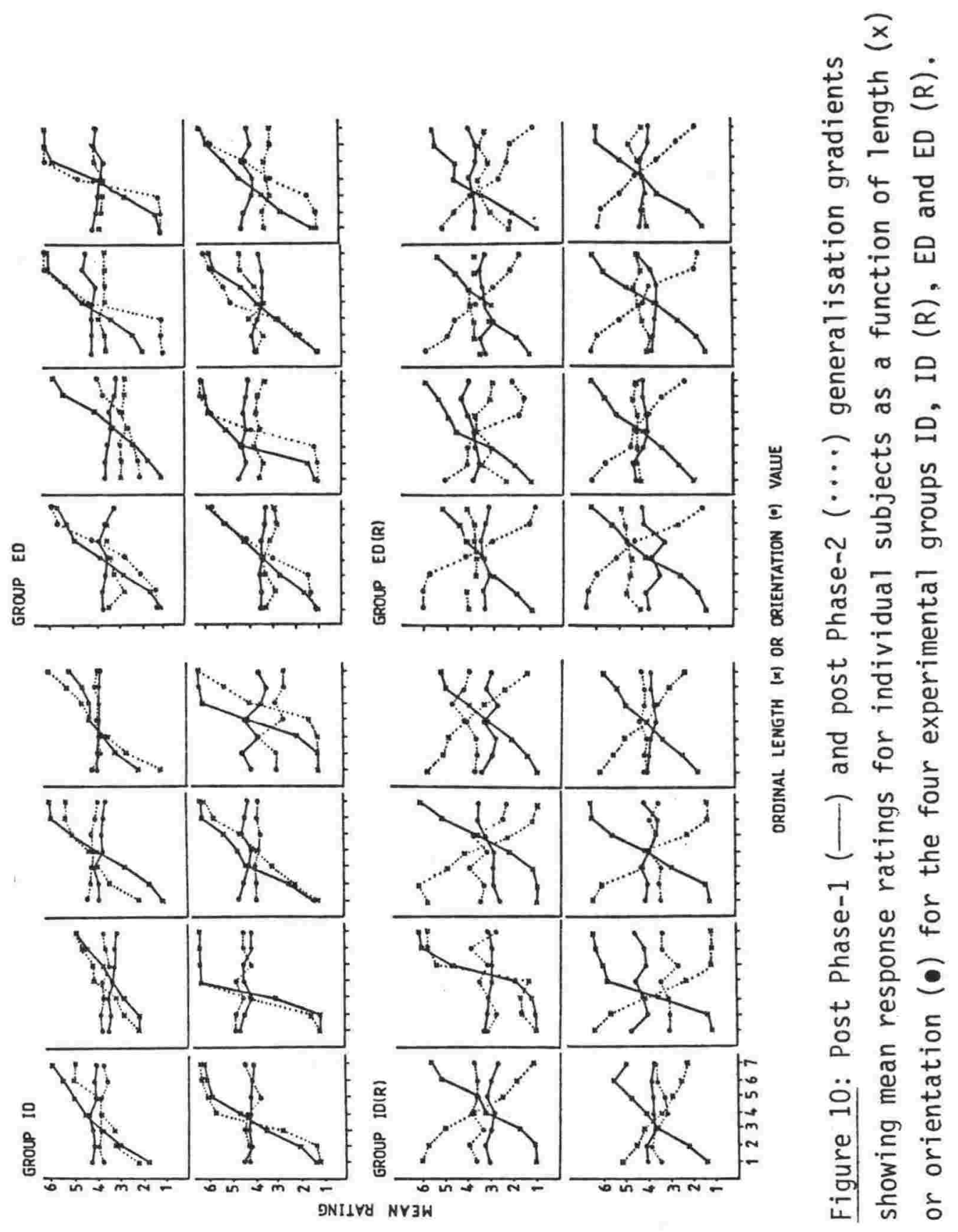


in Phase-1 and remained relevant in Phase -2 retains dimensional stimulus control. The gradients for the ID and ID (R) groups have the same ogival form. However, while the ID has its maxima at $20 \mathrm{~mm}$, the ID (R) group has its maxima at $8 \mathrm{~mm}$, the value adjacent to the new training stimulus for the Phase-2 reversal.

The ID (R) group, in addition to performing the reversal within the same number of trials as its non-reversal counterpart, also exhibits the same degree of dimensional stimulus control as the nonreversal ID group, except that control is by the new, reversed training stimuli. Analysis of variance examining the effects of Type of Training (ID vS ID (R)) $x$ Dimension, shows no significant difference for Type of Training where mean ratings to training stimuli are compared $(F(1,14)<1)$. The group mean data (Figure 9 (ii) is representative of the overall responding in this group, except that the gradients tend to be somewhat smoother. The individual data for each group is shown in Figure 10, and apart from some small idiosyncracies, all subjects show the same order of control after training to criterion, within a group.

The ED and ED (R) groups took the same number of trials to reach criterion. Although the training stimuli $\left(25^{\circ}\right.$ : 'yes' and $65^{\circ}$ : 'no') for the $E D(R)$ group were reversed when compared to the ED group, this was a "pseudo-reversal" compared to the "truereversal" of the ID and ID (R) groups. Phase-1 training for the ED and ED (R) groups was with the length cues differentially reinforced and the orientation cues non-differentially reinforced. 
78.

Therefore, there was no advantage for either the ED transfer group $\left(65^{\circ}\right.$ : 'yes' and $25^{\circ}$ : 'no') or the ED (R) transfer group (25': 'yes' and $65^{\circ}$ : 'no'). After Phase-2 criterion was reached, groups ED and ED (R) exhibit no difference in dimensional stimulus control by orientation, except that the particular cues on the dimensions control maximal and minimal responding.

\section{DISCUSSION}

The comparison between reversal and non-reversal shifts was important in view of the possibility that dimensional control in the ID groups could have been mediated by cue-specific transfer. That is, the faster transfer by the ID group could be attributed to stimulus generalisation from Phase-1 to Phase-2 training stimuli. However, the results have clearly demonstrated that the rapid transfer from Phase-1 to Phase-2 in the ID shift groups was independent of the specific stimulus values used, and that the transfer was therefore dimensional in nature. The result is consistent with two-process theory (Lovejoy, 1968: Sutherland \& Mackintosh, 1971) in that responses to specific cues were dissociated from attention to dimensions. That is, dimensional stimulus control was the same for Reversal and Non-reversal conditions where specific cues were reversed.

Further, the ED versus ID shift comparison confirmed the result of Experiment 1. In particular, the ID (R) and ED (R) groups required similar numbers of trials to criterion and exhibited the same extent of dimensional stimulus control as did their ID and ED counterparts. 


\section{EXPERIMENT 3}

It was established in Experiment 1 that strong stimulus control was exerted by the relevant dimension of the transfer task for both the ID and ED shift irrespective of which dimension was used for training or transfer. Results from Experiment 2 demonstrated further that the speed of transfer in the ID shift and degree of control by dimension was not solely attributable to stimulus generalisation. In both these experiments dimensional stimulus control was assessed after the Phase-2 discrimination had been well learned and a criterion performance for each subject had been reached.

Ray and Sidman (1970) have stressed the importance of being able to measure changes in stimulus control as they occur. Controlling relations can then be identified and subsequently maintained or eliminated. Ray (1971) describes attention as a phenomenon which is "famous for its instability" and requires an approach different from typical static analyses that are inadequate for monitoring change. Functional analyses, Ray (1971) stated, fulfil this role.

Heinemann et a1. $(1969,1970,1972)$ performed a series of experiments which examined stimulus control following discrimination tasks. They found that after training pigeons to discriminate between two intensities, the generalisation gradients had the form of normal ogives, as in the present experiments. In Heinemann et al.'s $(1969,1970,1972)$ experiments, all gradients 
80.

were obtained after the dsicriminations had been well learned. A subsequent experiment by Heinemann and Avin (1973) demonstrated how the development of stimulus control can be traced over time. They examined the changes that these gradients underwent during the course of training, by administering generalisation tests after every three days of training. Gradients changed from flat, chance-level functions between days $1-3$, to the same steep, sigmoidal gradients that characterised criterion training, after between 28 - 54 days.

Experiment 3 was designed to examine the development of control by the new relevant dimension in the ED shift over the course of the Phase-2 discrimination. The procedures for Experiments 1 and 2 were repeated with generalisation tests concluded after different numbers of Phase-2 trials.

METHOD

Subjects were 80 undergraduates who were randomiy assigned to ten experimental groups, with four males and four females in each group. The apparatus and general procedural conditions were the same as those used for the ID and ED shift groups in Experiments 1 and 2 .

Phase-1 discrimination training. For all ten groups the same discrete trials procedure was followed as for ID (L) and ED (L) groups in Experiment 1. Four training stimuli were presented equally often in a random order over 24 training trials. 'Yes' ratings were required to $20 \mathrm{~mm}$ lines and 'no' ratings were required 
to $8 \mathrm{~mm}$ lines, independently of line orientation $\left(15^{\circ}\right.$ or $\left.75^{\circ}\right)$.

Phase-2 discrimination training. Phase-2 discrimination training followed Phase-1 training without a break in the procedure. There were ten transfer conditions. The ID and ED transfer conditions differed according to whether there were $0,8,12,16$ or 20 trials in Phase-2 training (Table 7). (The number of trials in each condition was determined by the result that criterion learning in Experiment 1 was reached in the ED shift after approximately 24 trials). For the ID groups 'yes' ratings were required to $18 \mathrm{~mm}$ lines and 'no' ratings were required to $10 \mathrm{~mm} 1$ ines, independently of orientation $\left(65^{\circ}\right.$ or $\left.25^{\circ}\right)$. For the ED groups, 'yes' ratings were required to $65^{\circ}$ line orientations.

Generalisation testing. A generalisation test was administered directly following Phase-1 training for groups ID (0 trials) and ED (0 trials) or following the number of Phase-2 training trials for the remaining groups specified in Table 7. The testing procedure was identical to that used in Experiments 1 and 2, and involved 98 trials during which all combinations of line length and orientation were presented.

\section{RESULTS AND DISCUSSION}

Mean correct responses over the trials of the Phase-2 discrimination are plotted in Figure 11 for the different groups. The contrast in speed of transfer between the ID shift and ED shift groups is evident. The ID groups maintained accurate responding 
TABLE 7

Experimental conditions in Experiment 3 .

\begin{tabular}{lll}
\hline Group & $\begin{array}{l}\text { Type of } \\
\text { Shift }\end{array}$ & $\begin{array}{l}\text { Number of } \\
\text { Phase-2 Trials }\end{array}$ \\
\hline
\end{tabular}

$\begin{array}{llr}\text { ID (0 trials) } & \text { ID } & 0 \\ \text { ID (8) } & \text { ID } & 8 \\ \text { ID (12) } & \text { ID } & 12 \\ \text { ID (16) } & \text { ID } & 16 \\ \text { ID (20) } & \text { ID } & 20\end{array}$

ED (0 trials) ED 0

ED (8) ED 8

ED (12) ED 12

ED (16) ED 16

ED (20) ED 20



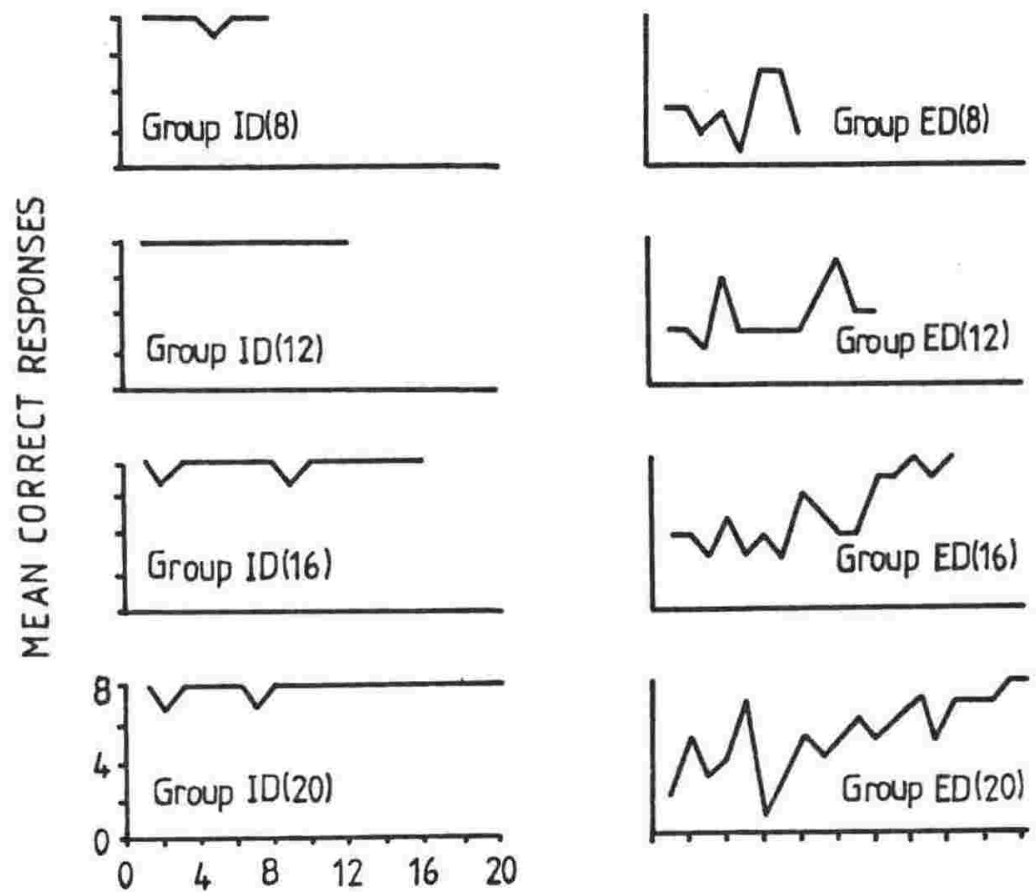

TRIALS

Figure 11: Number of correct responses per group as a function of the number of trials of either intradimensional [ID (8), (12), (16), (20)] or extradimensional [ED (8), (12),(16), (20)] Phase-2 discrimination training. 
throughout the Phase-2 discrimination, whereas ED groups acquired the new discrimination relatively slowly. Figure 11 shows that as the number of trials progressed over the different ED groups, there was a systematic improvement in performance by the 20 trials of Phase-2 training, (the mean criterion performance for ED groups in Experiments 1 and 2 being 24.34).

Generalisation gradients were plotted in the same way as in Experiment 1. Figure 12 shows the post-Phase-2 generalisation gradients for all ID and ED groups. The gradient for the ID (0) group demonstrates that the relevant dimension (length) in Phase-1 discrimination had gained dimensional stimulus control in that mean ratings varied systematically with length, but not with orientation (the irrelevant dimension). The mean ratings for this group had maxima at the stimulus value $20 \mathrm{~mm}$ requiring 'yes' ratings in prior discrimination training. Functions were flat for the irrelevant dimension, indicating absence of control by that dimension. The gradients for the remaining ID groups exhibit no change over trials. The ID groups show no change as a function of Phase-2 trials. That is, the ID gradients uniformly exhibit strong control by length and absence of control by orientation (the irrelevant dimension). Table 8 summarises separate two-way analyses of variance conducted within groups ID (8), ID (12) ID (16) and ID (20), confirming control, or lack of control, by the two dimensions. Two further analyses of variance (Keppel, 1982, p.438), make a between groups (0, 8, 12, 16 , 20 trials) comparison of the effect of either the length or the orientation dimension (Appendix I, Table 1). The results of these 


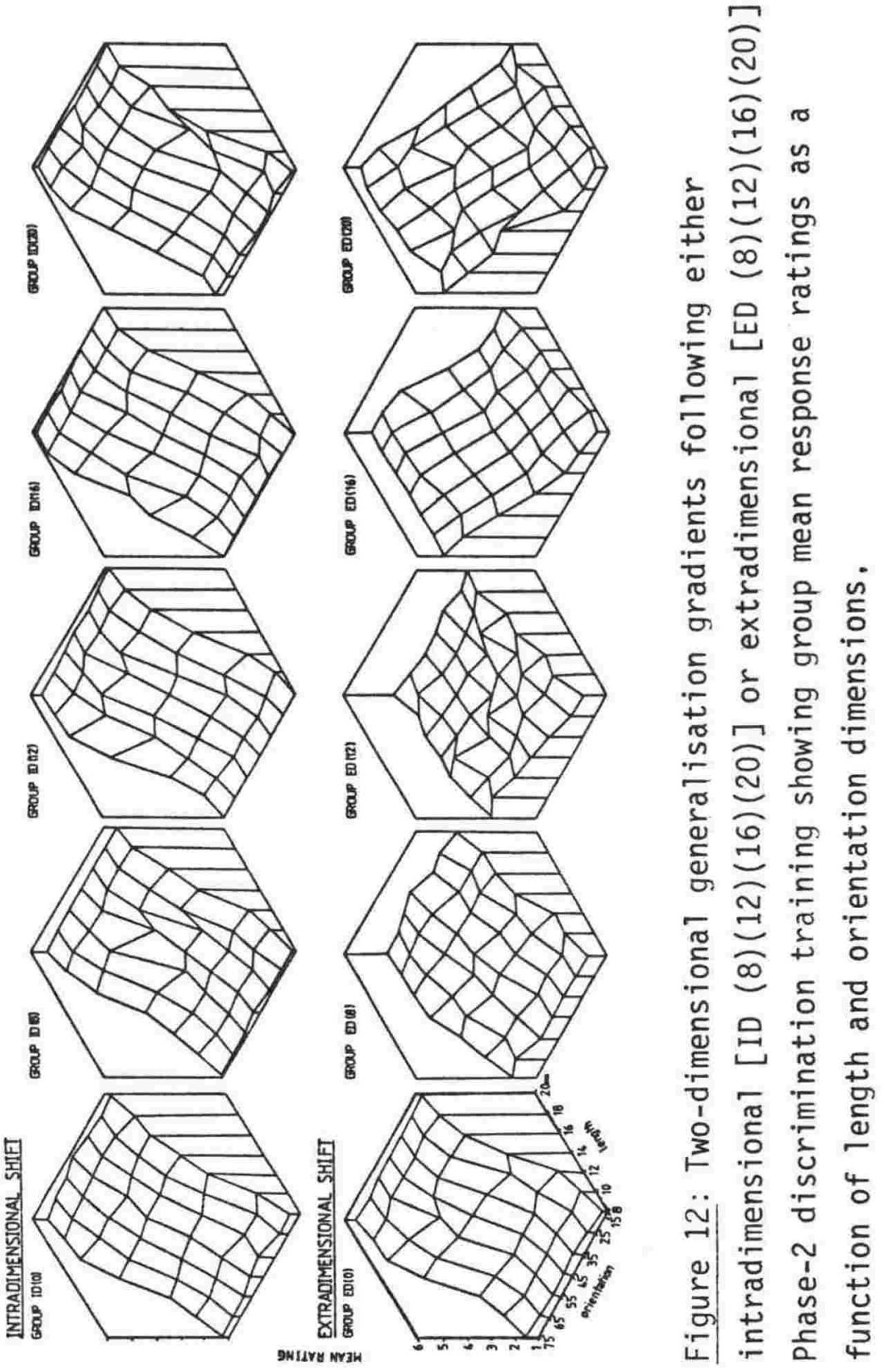


86.

\section{TABLE 8}

Summary of two-way analyses of variance to show effects

within groups of the relevant and irrelevant dimensions over trials $(8,12,16$ or 20$)$ of intradimensional or extradimensional Phase-2 discrimination training.

\begin{tabular}{|c|c|c|c|c|c|c|}
\hline CONDITION & DIMENSION & DF & MS & $\mathrm{F}$ & & \\
\hline ID (8) & $\begin{array}{l}\text { Length (REL) } \\
\text { Orient (IRR) }\end{array}$ & $\begin{array}{l}6,42 \\
6,42\end{array}$ & $\begin{array}{r}230.2 \\
0.9\end{array}$ & $\begin{array}{r}93.6 \\
1.4\end{array}$ & p & $\begin{array}{l}<.001 \\
\text { n.s. }\end{array}$ \\
\hline ID (12) & $\begin{array}{l}\text { Length (REL) } \\
\text { Orient (IRR) }\end{array}$ & $\begin{array}{l}6,42 \\
6,42\end{array}$ & $\begin{array}{r}178.3 \\
0.7\end{array}$ & $\begin{array}{r}44.3 \\
1.8\end{array}$ & $p$ & $\begin{array}{l}<.001 \\
\text { n.s. }\end{array}$ \\
\hline ID (16) & $\begin{array}{l}\text { Length (REL) } \\
\text { Orient (IRR) }\end{array}$ & $\begin{array}{l}6,42 \\
6,42\end{array}$ & $\begin{array}{r}242.7 \\
0.4\end{array}$ & $\begin{array}{c}48.6 \\
<1\end{array}$ & p & $\begin{array}{l}<.001 \\
\text { n.s. }\end{array}$ \\
\hline ID (20) & $\begin{array}{l}\text { Length (REL) } \\
\text { Orient (IRR) }\end{array}$ & $\begin{array}{l}6,42 \\
6,42\end{array}$ & $\begin{array}{r}219.4 \\
0.8\end{array}$ & $\begin{array}{r}121.2 \\
1.9\end{array}$ & $\mathrm{p}$ & $\begin{array}{l}<.001 \\
\text { n.s. }\end{array}$ \\
\hline ED (8) & $\begin{array}{l}\text { Length (IRR) } \\
\text { Orient (REL) }\end{array}$ & $\begin{array}{l}6,42 \\
6,42\end{array}$ & $\begin{array}{r}52.7 \\
1.5\end{array}$ & $\begin{array}{c}19.9 \\
<1\end{array}$ & $\mathrm{p}$ & $\begin{array}{l}<.001 \\
\text { n.s. }\end{array}$ \\
\hline ED (12) & $\begin{array}{l}\text { Length (IRR) } \\
\text { Orient (REL) }\end{array}$ & $\begin{array}{l}6,42 \\
6,42\end{array}$ & $\begin{array}{l}8.2 \\
0.5\end{array}$ & ${ }_{<}^{1.5}$ & & $\begin{array}{l}\text { n.s. } \\
\text { n.s. }\end{array}$ \\
\hline ED (16) & $\begin{array}{l}\text { Length (IRR) } \\
\text { Orient (REL) }\end{array}$ & $\begin{array}{l}6,42 \\
6,42\end{array}$ & $\begin{array}{r}2.8 \\
90.6\end{array}$ & $\begin{array}{r}1.9 \\
13.1\end{array}$ & & $\begin{array}{l}\text { n.s } \\
<.001\end{array}$ \\
\hline$E D(20)$ & $\begin{array}{l}\text { Length (IRR) } \\
\text { Orient (REL) }\end{array}$ & $\begin{array}{l}6,42 \\
6,42\end{array}$ & $\begin{array}{r}3.6 \\
99.6\end{array}$ & $\begin{array}{r}1.4 \\
25.3\end{array}$ & & $\begin{array}{l}\text { n.s. } \\
<.001\end{array}$ \\
\hline
\end{tabular}


analyses show that control by neither length nor orientation changes for the ID shift over trials of Phase-2 discrimination training. Control by the relevant dimension was therefore established in Phase-1 and maintained throughout Phase-2 discrimination training.

In addition to the ID groups, two further ID (reversal) groups were run and their performance measured after 12 and 20 trials of intradimensional (reversal) Phase-2 discrimination training. The procedure and results for these two groups appear in Appendix II. Appendix II Figure 1 clearly shows that, as for their ID counterparts (ID (12) and ID (20): Figure 12), the ID (R) groups showed strong control by the length dimension after both 12 and 20 trials of Phase-2 training.

In contrast, gradients for the ED groups (Figure 12) changed markedly as a function of the number of training trials in the Phase-2 discrimination. Data for the four ED groups ( 8 - 20 trials) was submitted to a three-way analysis of variance with repeated measures on the factors of length and orientation (Winer, 1971, p.540). Results of this analys is are summarised in Table 9. Significant two-way interactions between number of transfer trials and orientation, number of transfer trials and length and not between length and orientation confirmed the changes in stimulus control over trials manifest in Figure 11. Two further two-way analyses of variance (Keppel, 1982, p.438) make a between groups $(0,8,12,16$ and 20 trials) comparison of the effect of either the length or the orientation dimension (Appendix I, Table 2). The 
88.

\section{TABLE 9}

Summary of three-way analysis of variance to show between group effects of number of trials $(8,12,16$ or 20) of extradimensional Phase-2 discrimination training as a function of the length and orientation dimensions.

Source

DF

MS

F

\begin{tabular}{|c|c|c|c|c|}
\hline TRIALS $(8,12,16,20)$ & 3,28 & 0.7 & $<1$ & n.s. \\
\hline ORIENTATION $\left(75^{\circ} \ldots 15^{\circ}\right)$ & 6,168 & 116.5 & 29.3 & $p<.001$ \\
\hline TRIALS $\times$ ORIENTATION & 18,108 & 26.0 & 6.5 & $p<.001$ \\
\hline LENGTH $(20 \mathrm{~mm} \ldots .8 \mathrm{~mm})$ & 6,168 & 45.0 & 107.1 & $p<.001$ \\
\hline TRIALS $\times$ LENGTH & 18,108 & 7.7 & 18.4 & $p<.001$ \\
\hline LENGTH $\times$ ORIENTATION & 36,1008 & 0.6 & $<1$ & n.s \\
\hline $\begin{array}{l}\text { TRIALS } x \text { LENGTH } x \\
\text { ORIENTATION }\end{array}$ & 108,1008 & 0.5 & $<1$ & $n$ \\
\hline
\end{tabular}


89.

results of these analyses show that the number of trials was a significant factor in determining control by either dimension. Specifically, as training trials increased over ED shift groups, control by orientation was successively sharpened and control by length was attenuated.

Figure 12 shows that immediately after Phase- 1 training the gradient for Group ED (0) indicates control by the Phase-1 relevant dimension, length, and no control by orientation. After eight trials of ED discrimination, the gradient for group ED (8) exhibits no evidence of control by orientation, the Phase-2 relevant dimension. After 12 trials of ED discrimination, control by length is further attenuated and there is still no apparent control by orientation. By 16 and 20 trials of ED discrimination, attenuation of control by length is complete and control by orientation continues to develop. The gradient for group ED (20) is sharpest along the orientation dimension. Separate two-way analyses of variance conducted for groups ED (8), ED (12), ED (16) and ED (20) and summarised in Table 8, confirm the conclusions drawn from the post-Phase-2 generalisation gradients.

The changes in dimensional stimulus control for the group gradients in Figure 12 are also clearly evident in the gradients for the individual subjects in the different groups. In Figure 13 mean ratings are plotted for orientation averaged over all lengths and for length averaged over all orientations. For each subject in the four ID groups, gradients along the relevant dimension were 


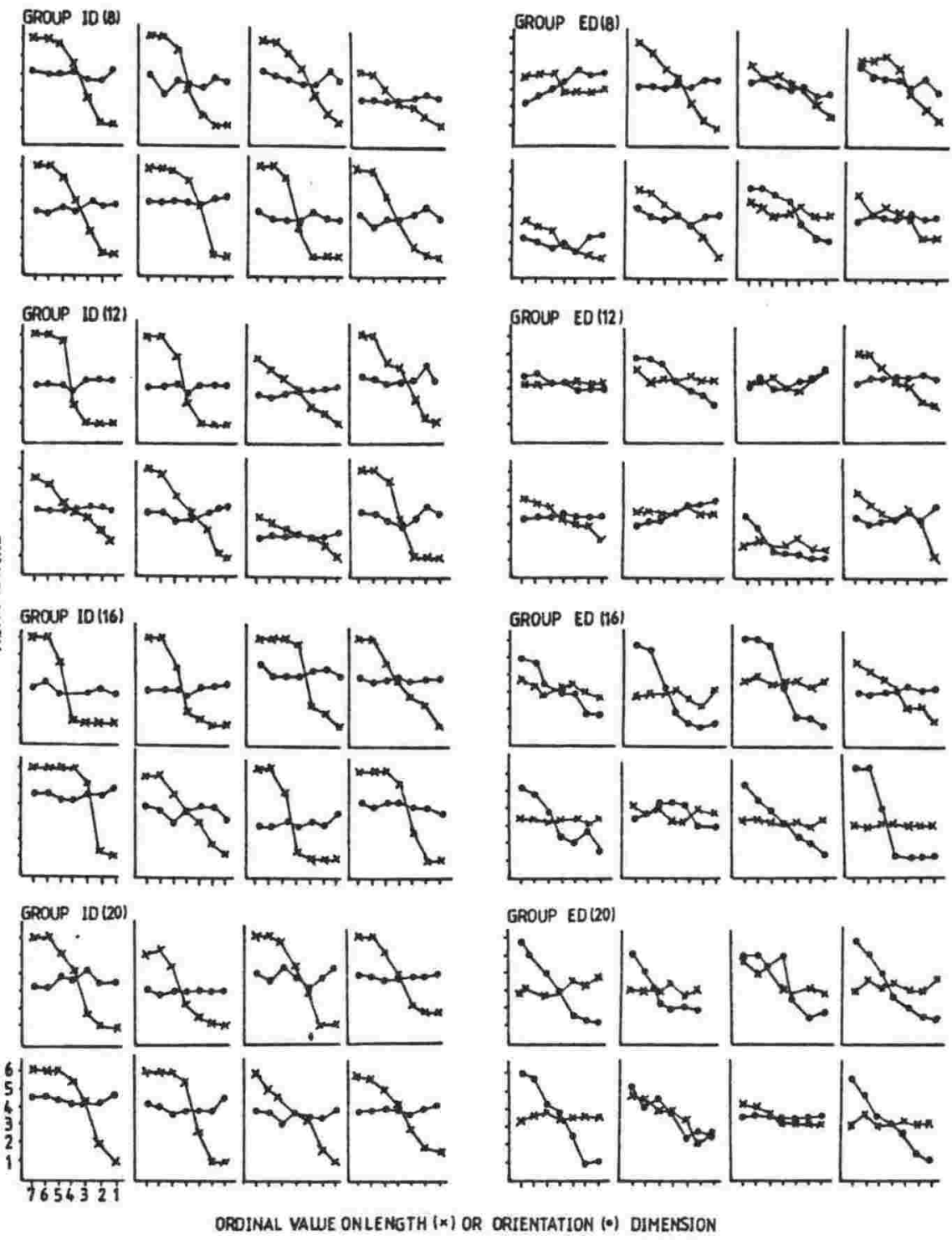

Figure 13: Post Phase-2 generalisation gradients showing mean response ratings for individual subjects as a function of length $(x)$ or orientation (o) following either intradimensional [ID (8)(12)(16)(20)] or extradimensional [ED (8)(12)(16)(20)] discrimination training. 
steep and along the irrelevant dimension, they were flat. For subjects in the ED groups, control by orientation increased as a function of training trials while control by length decreased.

The result of Experiment 3 shows that control by the new relevant dimension in the Phase-2 discrimination involves a systematic weakening of control by the dimension that was relevant in Phase-1 and an emergence of control by the Phase-2 relevant dimension in the ED shift. The present results provide some evidence to show why an ID-ED shift difference is obtained. In the ID shift the relevant dimension has gained control in Phase-1 and that control persists throughout Phase-2, since the Phase-1 relevant dimension remains relevant in Phase-2. Rapid acquisition of the new task in the ID shift as evidenced in all ID and ID (reversal) groups emphasises the 'floor effect' that obtains in this transfer task.

In the ED shift condition, the previously irrelevant dimension has no control by the end of Phase-1, but since it is now the relevant dimension in Phase-2, it must gain control over the course of the Phase-2 training. Experiments $4-6$ are therefore going to examine specific variables that may differentially affect the extradimensional shift and may increase the rate of acquisition of that shift under different conditions. Such variables include the type of Phase-1 training, the type of Phase-1 and Phase-2 stimulus arrays and the nature of the testing procedure itself. 


\section{EXPERIMENT 4}

Experiment 4 asks whether training with the relevant dimension in Phase-1 facilitates or retards training with the new relevant dimension in the extradimensional shift of Phase-2. Theories of selective attention and attentional enhancement (or general attentiveness) make different predictions in this regard. These theories have previously been examined in studies of extradimensional discrimination and generalisation where single dimensions are present in Phase-1 and Phase-2 (Gray \& Mackintosh, 1973: Hogg \& Evans, 1975, 1978: Honig, 1969: Thomas et a1., 1970: Turner \& Mackintosh, 1972). Such studies indicate that discrimination training can have a number of different effects. A general effect of discrimination training is that it neutralises incidental cues in the environment (Mackintosh, 1974), and thereby minimises control by those stimuli. Other general effects are that differential training in Phase-1 leads to a sharpening of stimulus control and hence a steeper generalisation gradient in Phase-2, whereas nondifferential training leads to a weakening of stimulus control (Thomas et al., 1970).

A number of experiments have compared the effects of differential and non-differential training on ED transfer. Using an ED procedure Honig (1969) found that True-discrimination (differential) training with wavelengths produced steeper generalisation gradients along the orientation dimension than did Pseudo-discrimination (non-differential) training with wavelength. Thomas et al. (1970) extended this finding to the case where compounded cues were used in training. Again, 
True-discrimination (TD) training on one dimension produced steeper gradients on an orthogonal dimension compared to Pseudo-discrimination (PD) training. Thomas et a1. (1970) argued that discrimination training produces a "set to discriminate" and enchances attention to other stimulus dimensions. Results from various ED procedures offer strong support for attentional enhancement (Robles, Thomas \& Newlin, 1980)

These findings challenged theories of selective attention which predict that, as an inverse relationship exists between dimensions in an ED paradigm, TD training will reduce the amount of control by an irrelevant dimension, relative to PD training. One of the few results supporting selective attention was reported by Gray and Mackintosh (1973) who tested the prediction using a discrete-trial procedure. They found that control by orientation was sharper following PD training on a tone discrimination than following TD training on the tone dimension, and concluded that Thomas et al.'s (1970) finding was limited to free-operant procedures. It is possible that "stimuli from the same modality (such as visual stimuli like line and colours) are subject to attentional enhancement, whereas stimuli from different modalities are not" (Honig \& Urcuioli, 1981, p.436), but there is no evidence that attentional enchancement in the ED procedure is more reliable in free-operant than in discretetrial procedures. Hogg and Evans (1975) trained ESN children in a discrete-trial ED procedure and demonstrated that TD training to orientation produced better control by hue than PD training to orientation. Hall and Channell (1980) reported evidence consistent 
with attentional enhancement in their ID versus ED shift experiments with pigeons. They concluded in a later study (Hall \& Channell, 1985) that "nondifferentially reinforced pre-exposure can cause stimuli to lose associability" and thus lead to a "reduction in the power of the stimulus to attract attention" (Hall \& Channel1, 1985, p.294).

In the present experiments, the relevant dimension involves a true discrimination (TD) whereas the irrelevant dimension involves a pseudo-discrimination (PD). What is the influence of TD with the relevant dimension in Phase-1 on performance in Phase-2? Experiment 4 was designed to investigate the development of control by the Phase-2 relevant dimension (orientation) following TD training with line length in Phase-1 compared to PD training along line length in Phase-1. According to theories of selective attention, TD training in Phase-1 would retard the development of control by the relevant dimension in Phase-2 (orientation), because it increases the probability that subjects will attend to the Phase-1 relevant dimension, length. If, however, values on the length or orientation dimensions in Phase-1 are not correlated with reinforcement (PD), neither dimension should gain control. That is, the probability of attending to either dimension following Phase-1 PD training should be at a chance level, and, therefore there would be no subsequent impairment in Phase-2 of control by the relevant dimension (orientation) by previous control by length. In Experiment 4, subjects were trained in a Pseudo-discrimination task in Phase-1 
shift'. Gradients following 0, 12 or 20 trials of ED training in Phase-2 were compared to the gradients obtained in Experiment 3 for the ED (0), ED (12) and ED (20) groups for which Phase-1 involved a true discrimination.

\section{METHOD}

Subjects were 24 undergraduates who were allocated to one of three groups. Each group comprised equal numbers of males and females. Apparatus was the same as that used in Experiments 1 to 3. The general procedure involved Phase-1 and Phase-2 discrimination followed by a generalisation test, conducted in the same way as in Experiment 3 .

Phase-1: Pseudo-discrimination training. The PD training used the same procedure as for Phase-1 discrimination training in the previous experiments, but the experimenter's feedback for responses to the stimuli were noncontingent as shown in Table 10. Specifically, the experimenter's "correct" and "incorrect" feedback was independent of the subjects" 'yes' and 'no' ratings and occurred equally often for both values of length and both values of orientation. Each subject received 24 trials of Phase-1 PD training.

Phase-2: Discrimination training. Phase-2 discrimination training was conducted as for the ED shift groups in Experiment 3, with orientation as the relevant dimension. Subjects received 0, 12 or 20 trials of Phase-2 discrimination training called PD (0), PD (12) and PD (20) groups. 
96.

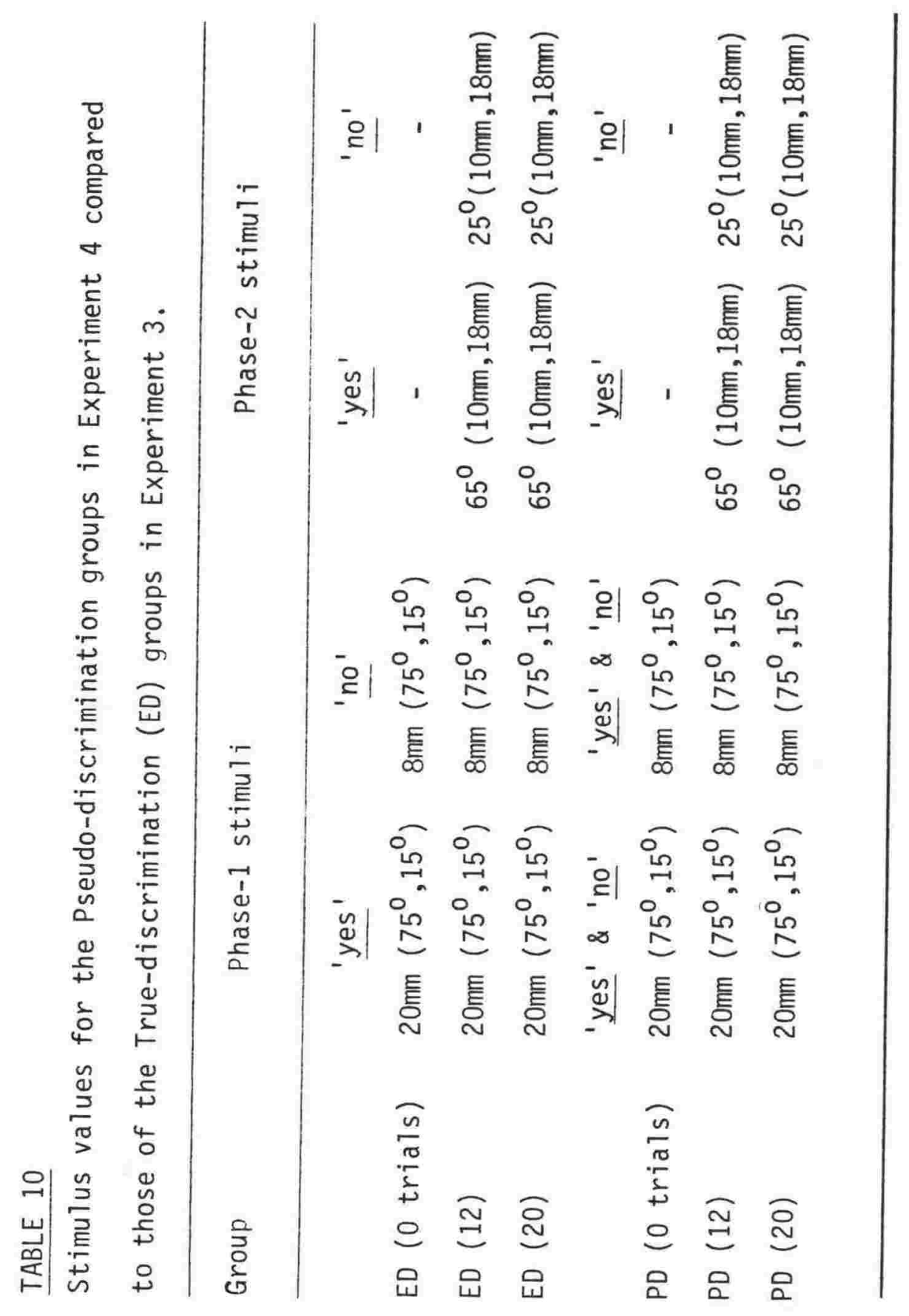


Generalisation testing. The PD (12) and PD (20) groups were administered generalisation tests conducted as in Experiments 1 - 3 after the pre-determined number of Phase-2 discrimination training trials, without a break in the procedure. The PD (0) group was administered a generalisation test immediately following PD training in Phase-1.

\section{RESULTS}

Individual and group results from the three PD groups were compared to those for ED groups ED (0), ED (12) and ED (20) from Experiment 3. Correct responses over the trials of Phase-2 discrimination training are plotted in Figure 14 for both PD and ED shift groups and the contrast in speed of transfer for each condition is evident. As the number of trials progressed over ED transfer there was a systematic improvement in performance. This improvement was not evident after 20 trials of PD transfer.

The PD (0) group was given a generalisation test immediately following PD training. Group mean data (Figure 15 (i)) and individual data (Figure 16) for the PD (0) group show no control by either orientation $(F<1)$ or length $(F<1)$ dimensions. Individual gradients establish that lack of control by the orientation and length dimensions in Figure 15 (i) is not merely a result of averaging gradients for individual data, but accurately reflects control by these dimensions within individuals.

Data from the four groups PD (12), PD (20), ED (12) and ED (20) 

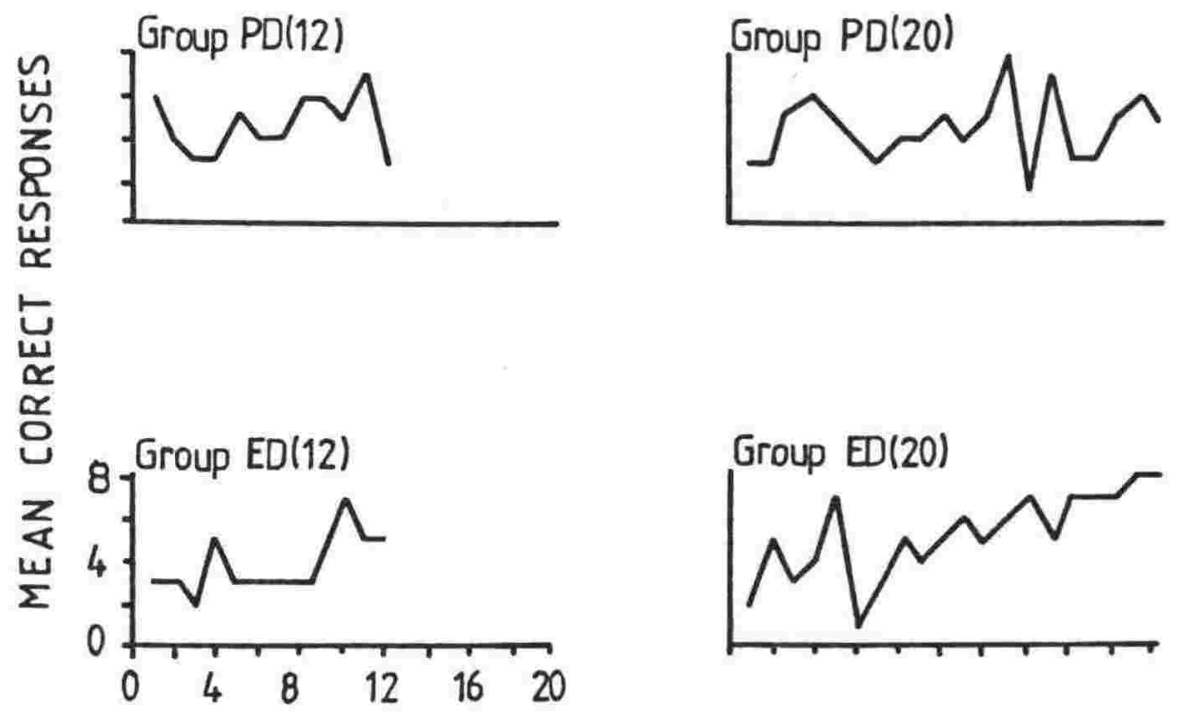

TRIALS

Figure 14: Number of correct responses per group as a function of the number of trials of Phase-2 discrimination training following 24 trials of either Phase-1 Pseudo-discrimination training [PD (12) and (20)] or Phase-1 True-discrimination training [ED (12) and (20)]. 
PSEUDO-DISCRIMINATION

(i)

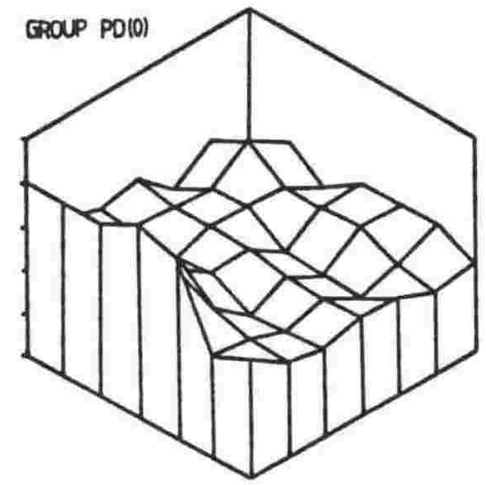

(ii) GRoup po(12)
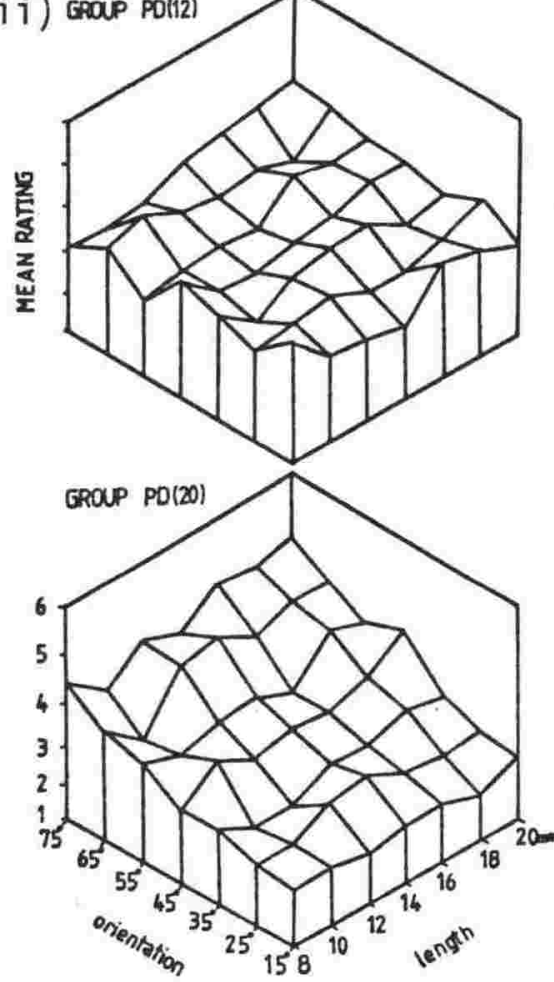

EXTRADIMENSIONAL SHIFT
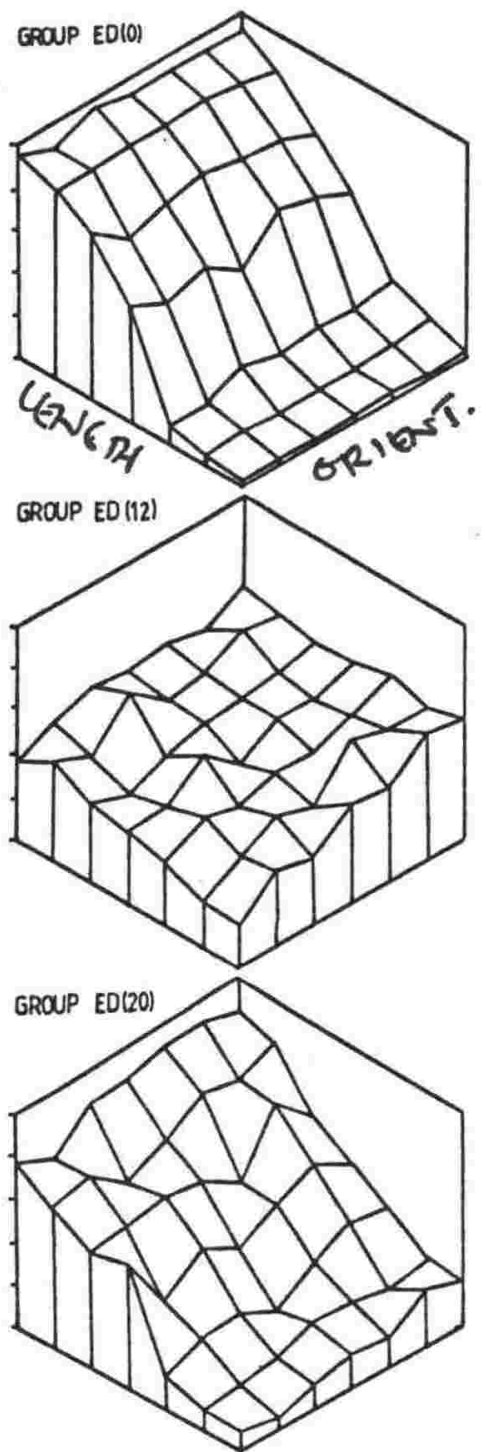

Figure 15: Two-dimensional generalisation gradients showing group mean response ratings as a function of length and orientation dimensions following Phase-2 discrimination training with orientation (relevant) and length (irrelevant). Groups PD (0), (12) and (20) received Phase-1 Pseudo-discrimination training and Groups ED (0), (12) and (20) received Phase-1 True-discrimination training with length (relevant) and orientation (irrelevant). 
100.
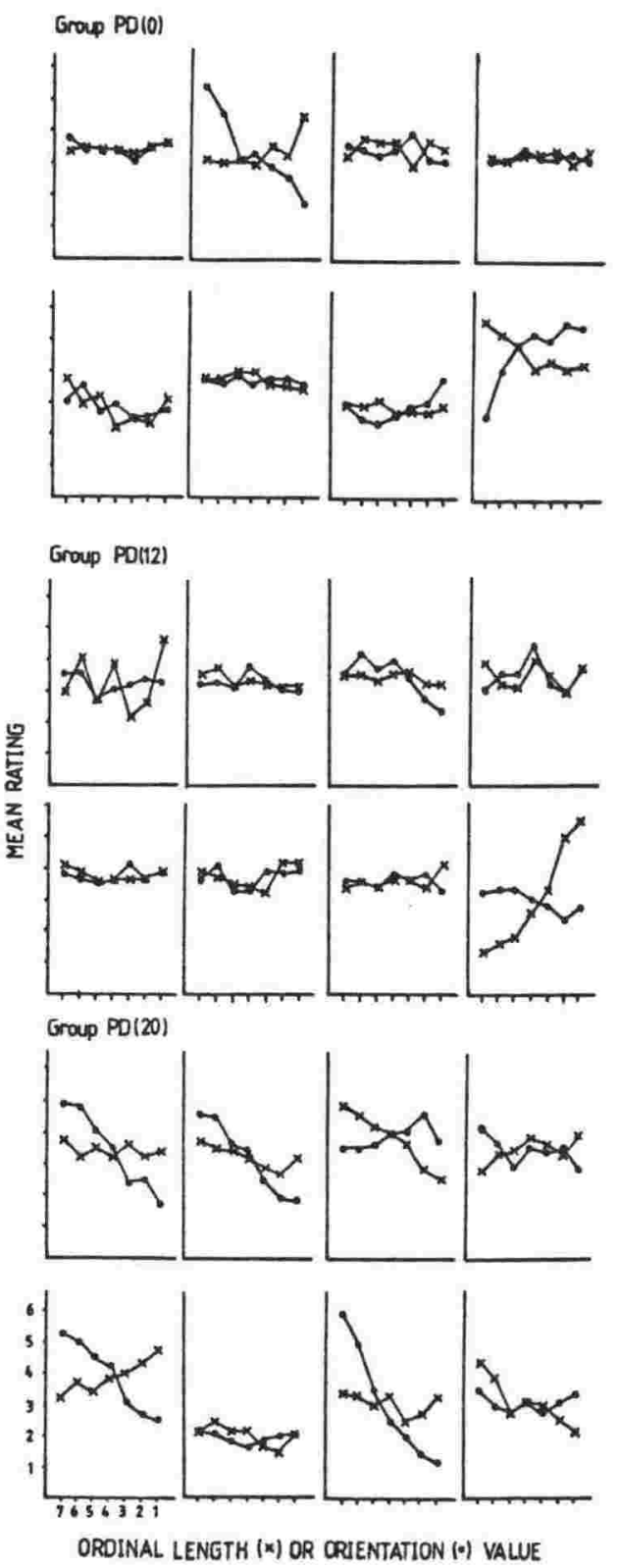

Figure 16: Generalisation gradients showing mean response ratings for individual subjects as a function of length $(x)$ and orientation ( $)$ following 0,12 or 20 trials of Phase-2 discrimination training with orientation (relevant) and length (irrelevant). Groups PD (0), (12) and (20) received Phase-1 Pseudo-discrimination training. 
(Figure 15 (ii))were submitted to a three-way analysis of variance (Winer, 1982) for repeated measures on the factor of orientation (the relevant dimension). These results are summarised in Table 11. Main effects of Array (TD versus PD), and Trials (12 versus 20) were not significant. However, two-way interactions between Array and Dimension, Trials and Dimension and a three-way interaction between Array, Trials and Dimension revealed a more careful look at the overall picture was required. Separate two-way analyses of variance for ED (12) versus PD (12) and for ED (20) versus PD (20) confirmed that after 20 trials of Phase-2 discrimination the difference in control by orientation between ED and PD was greater than after 12 trials (refer Appendix I, Table 3). The number of trials was critical: at 12 trials there was no difference in control by the orientation dimension, but after 20 trials control by orientation in Phase-2 was stronger after ED than after PD training in Phase-1.

\section{DISCUSSION}

The present experiment set out to answer the question of whether development of stimulus control by the relevant dimension in the Phase-2 ED shift was retarded by the control established for the relevant dimension in the Phase-1 task. The mechanism for such an effect was presumed to be competition between dimensions in Phase-2, with residual control by the Phase-1 relevant dimension retarding the acquisition of control by the Phase-2 relevant dimension. Such an account is consistent with two-process theories (Sutherland \& Mackintosh: 1971). The results of Experiment 4 were directly 
102.

\section{TABLE 11}

Summary of three-way analysis of variance to show between group effects of Type of Training and Number of Trials as a function of Dimension for Groups PD (12) and (20), and Groups ED (12) and (20).

\begin{tabular}{lcccc} 
Source & DF & MS & F & \\
\hline TRAINING (TD vs PD) & 1,28 & 0 & $<1$ & $\mathrm{n} . \mathrm{s}$. \\
TRIALS (12 vs 20) & 1,28 & 0.1 & $<1$ & $\mathrm{n} . \mathrm{s}$. \\
DIMENSION $\left(75^{\circ} \ldots 15^{\circ}\right)$ & 6,168 & 10.3 & 93.2 & $\mathrm{p}<.001$ \\
TRAINING $\times$ TRIALS & 1,28 & 1.7 & $<1$ & $\mathrm{n} . \mathrm{s}$. \\
TRAINING $\times$ DIMENSION & 6,168 & 1.4 & 12.6 & $\mathrm{p}<.001$ \\
$\begin{array}{l}\text { TRIALS } \times \text { DIMENSION } \\
\text { TRAINING } \times \text { TRIALS } \times\end{array}$ & 6,168 & 6.6 & 60.3 & $\mathrm{p}<.001$ \\
DIMENSION
\end{tabular}


contrary to the possibility that the Phase-1 TD may retard the development of the Phase-2 ED discrimination. Instead, consistent with Honig's (1969) findings with pigeons and with attentional enhancement (Thomas, 1970), the Phase-1 TD may actually enhance the development of control by the new, relevant dimension in Phase-2.

Hogg and Evans (1975) compared the performance of severely retarded children on an ED task following both PD and TD training. They used a "Honig" type (1969) ED procedure which exposed subjects firstly to PD or TD training with orientations, followed by generalisation testing along the hue dimension in Phase-2. Comparing generalisation gradients on the hue dimension, they found strong stimulus control by hue following TD training with orientation, and no stimulus control by hue following PD training with orientation. They concluded that "exposure to the orientation stimuli without training differential responding is not sufficient condition for the occurrence of positive transfer to the second dimension" (Hogg \& Evans, 1975, p.218) and that the "crucial distinction rests in the association of differences between the stimuli on the orientation dimension with different response consequences" (1975, p.219).

In both experiments by Hogg and Evans (1975) and in Experiment 4, subjects in the PD and TD shift groups received equal exposure to the two training dimensions in Phase-1, but not equal reinforcement. Both the results from Hogg and Evans (1975) and Experiment 4 have shown the same pattern. Differential training on an extradimensional Phase-1 task (ED groups) produced positive transfer to a Phase-2 
discrimination relative to non-differential training in a Phase-1 task (PD groups). It is clear that some aspect of differential training in the ED task transferred to the second discrimination. In Experiment 4, having length relevant in Phase-1 (TD) facilitated the development of control by orientation relative to non-differential training on the length dimension (PD).

Hogg and Evans (1975) did not present the two dimensions used in the discrimination tasks concurrently. The present experimental design, which parallels the ED shift design more commonly used in the ID-ED comparison with adult, human subjects, is complicated by the fact that two dimensions are presented simultaneously in both Phase-1, Phase-2 and testing arrays. The comparison is arranged in this manner to avoid confounding the attentional effects of the shift to the new discrimination with novelty effects.

Sutherland and Mackintosh (1971) have pointed out that because the subjects in both ID and ED transfer conditions have equal exposure to the stimuli on both dimensions, this leads to a confounding of two other effects in accounting for ID-ED shift differences. Where the ID shift is faster and leads to greater stimulus control by the Phase-2 relevant dimension than the ED shift, there are two alternative effects. Firstly, the presence of the previously relevant dimension in transfer (Phase-2) impairs the development of control by the new, relevant dimension in Phase-2. Secondly, if the relevant dimension in Phase-2 was previously irrelevant in Phase-1, subsequent control when it becomes relevant 
is retarded. This effect has been labelled "learned irrelevance" (Mackintosh, 1965).

With respect to the first alternative, Experiment 4 has shown that the presence of length did not impair control by orientation in Phase-2. In fact, relative to PD training, ED training improved control by the orientation dimension in Phase-2. Length was present and irrelevant in both TD and PD Phase-2.

The design of Experiment 4 does not allow an evaluation of the second alternative, that if the Phase-2 relevant dimension was irrelevant in Phase-1, subsequent control will be retarded. In both TD and PD groups the Phase-2 relevant dimension, orientation, was irrelevant. In order to seek support for the hypothesis of "learned irrelevance", Experiment 5 examined the effects of varying the irrelevant dimension in training and transfer procedures on the rate at which the Phase-2 discrimination was learned. Conditions where values on the irrelevant dimension were varied during training and/or transfer tasks were compared to conditions where the irrelevant dimension was held constant. It could then be seen whether the irrelevant dimension in Phase-1 impairéd control by that dimension (now relevant) in the Phase-2 task. 


\section{EXPERIMENT 5}

Experiments 1, 2 and 3 demonstrated the well-established finding of an ID-ED shift difference for humans (Harrow, 1964: Kennedy \& Gersten, 1976: LeBow \& Tritt, 1971: Shanab \& Yasin, 1979). The ID-ED shift effect may depend, however, on whether values of the irrelevant dimension change within trials.

Ozioko and May (1977) investigated the effects of irrelevantdimension variability with adults in order to replicate Esposito's (1975) general finding that children learned the ED shift slower when the irrelevant dimension was varied within trials. Ozioko and May (1977) found the same effect with adult students. The ID shift was learned faster than the ED shift when the irrelevant dimension was varied within trials, but there was no ID-ED difference when the irrelevant dimension varied between trials. In other words, when only one value of the irrelevant dimension was used on any particular trial, the ID-ED shift difference was attenuated.

Trabasso, Deutsch and Gelman (1966) studied the effects of attention to the irrelevant dimension on the learning rates of the ID and ED shift with young children. Trabasso et a1. used constantirrelevant conditions in both phases of the procedure. When stimulus dimensions were patterns (two-dimensional shapes and colours) they found that there was no significant difference between numbers of errors for the ID and ED shift groups (Mean Transformed Error: $I D=1.89, E D=2.76, t-1.38 d f=31$ ). However, an experiment reported in the same paper replicating the procedure with three- 
107.

dimensional coloured blocks of varying sizes, produced the usual ID-ED shift performance difference.

In terms of two-process accounts, Fisher and Zeaman (1973), Sutherland and Mackintosh (1971) and Lovejoy (1968) would argue that cue differences act on the probability of attending to a dimension, that is, the first of the two processes. Fisher and Zeaman (1973, p.220) summarise their prediction that "with no competition from irrelevant dimensions the remaining relevant dimensions (with variable cues...) control performance to about the same extent in both ID and ED conditions". For Lovejoy (1968), where the cue on the irrelevant dimension is constant-irrelevant, the probability of control by that dimension remains at its base-level distinctiveness or salience. This suggests that the ID-ED difference would be either eliminated or attenuated.

Experiment 4 illustrated that in the current successive paradigm the ED performance decrement relative to the ID shift may be attributable to one of two main factors: learned irrelevance, or the presence of the previously relevant dimension in Phase-2.

Experiment 5 was designed to demonstrate empirically whether either of these factors affected the ID-ED shift difference, by comparing transfer between combinations of constant-irrelevant and two-dimensional arrays. In the two-dimensional (2D) condition, the cues on the relevant dimension were permitted to vary and were associated with non-differential reinforcement. In the constant- 
108.

irrelevant (CI) condition the cues on the relevant dimension varied in the same way as for the 2D array and were associated with differential reinforcement, but the cue on the irrelevant dimension remained at a constant value and was randomly reinforced.

Subjects were transferred from either a CI Phase-1 discrimination task or 2D Phase-1 discrimination task (see Table 12). It was therefore possible to examine the acquisition of a discrimination on a dimension which had not been irrelevant in Phase-1 (ED:CI-2D), or the acquisition of a discrimination when the previously relevant dimension was removed (ED:2D-CI). These conditions were then compared to the previously used ED:2D-2D, where the two effects of Phase-1 and Phase-2 discriminations were confounded. They can further be compared to the ED:CI-CI condition where neither effect should have been present.

METHOD

Subjects were 72 undergraduates. Equal numbers of males and females were randomly assigned to each of the nine experimental groups. Table 12 summarises the conditions for eight groups. The ninth group is described below. The apparatus used and the general procedural conditions were the same as those in all previous experiments.

Phase-1 discrimination training. All subjects received 24 trials of either two-dimensional or constant-irrelevant Phase-1 discrimination 
109.

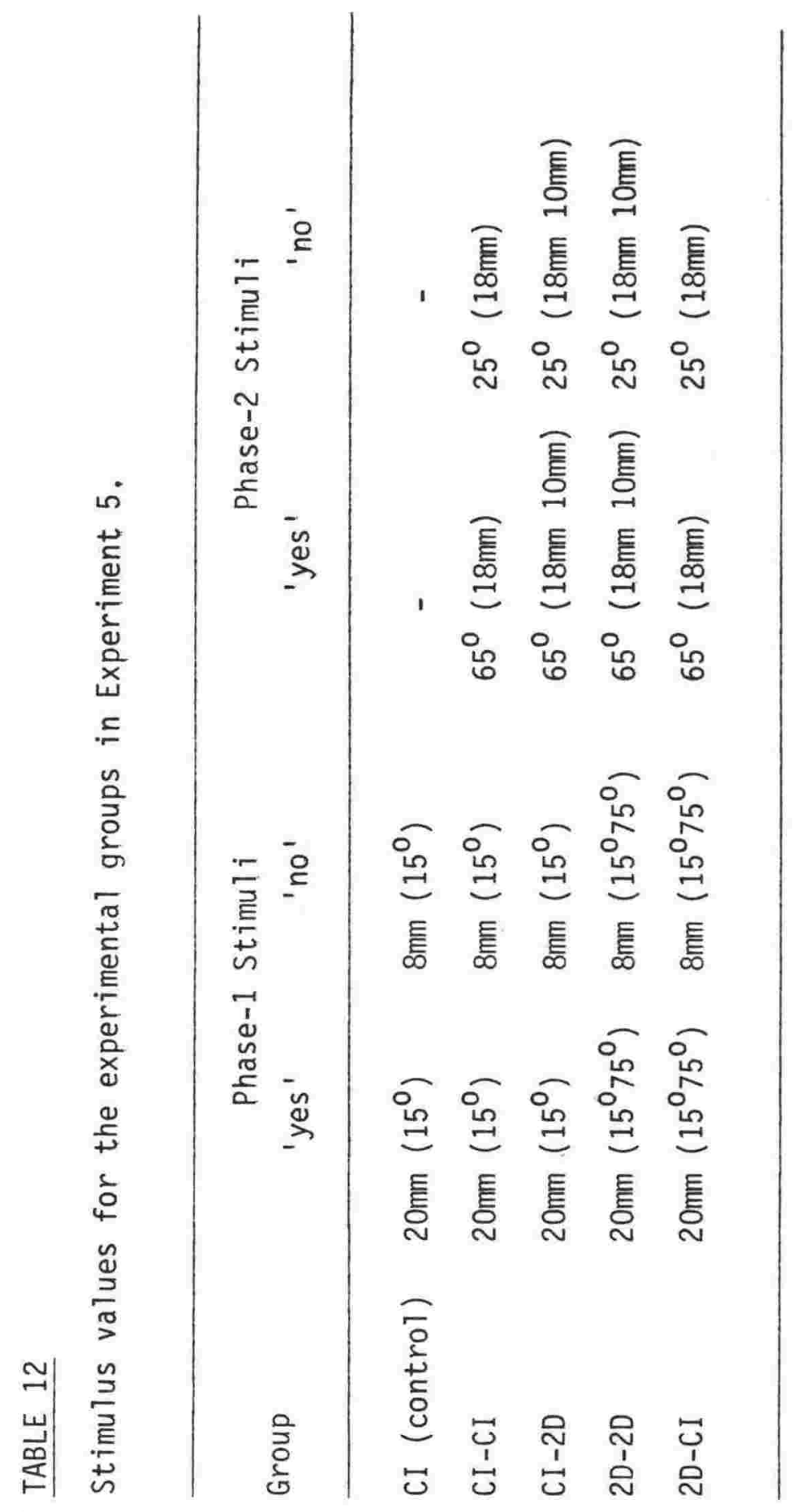


training. Table 12 shows how stimuli for the Phase-1 discrimination varied according to the condition to which the subjects were assigned. An additional eight subjects were run in a control group for the constant-irrelevant condition. These were given Phase-1 CI training only, followed immediately by a generalisation test.

Phase-2 discrimination training. Trials of Phase-2 discrimination training continued from Phase-1 training without any break in the procedure. Subjects transferred to one of two transfer conditions as specified in Table 12, two-dimensional or constant-irrelevant, and each subject received either 12 or 20 transfer trials as in Experiment 3, groups ED (12) and ED (20).

Generalisation testing. A generalisation test was administered to each subject following the pre-determined number of Phase-2 discrimination trials. The test combined seven values of orientation with seven values of length over 98 trials.

\section{RESULTS}

Control group.

Comparing group mean generalisation gradients for subjects following 24 trials of Phase-1 constant-irrelevant (Figure 17) and two-dimensional (Figure 5) training, it can be seen that the same order of control is exerted by the relevant dimension, line length. Data for the group CI (Control) shows a significant effect of length $(F(6,42)=47.12 \mathrm{p}<.001)$, no effect of orientation $(F<1)$ 
111.

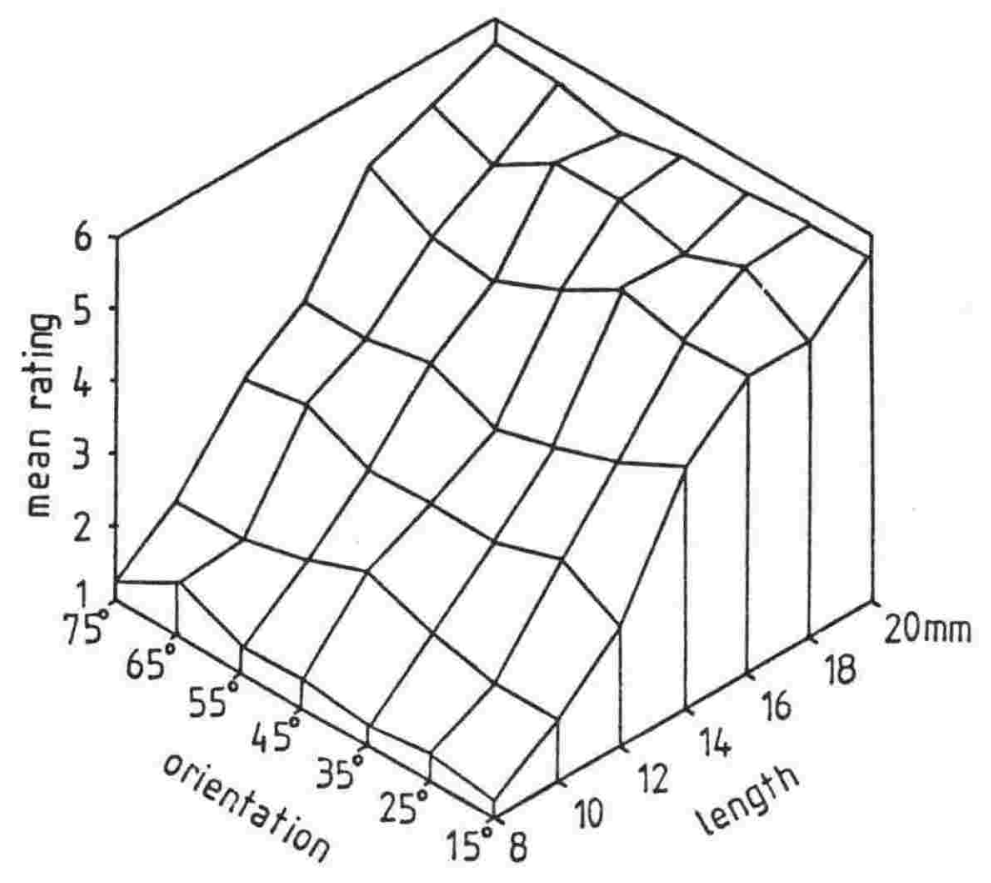

Figure 17: Post-Phase-1 two dimensional generalisation gradient showing mean response rating as a function of length and orientation dimensions following 24 trials of Phase-1 discrimination training with length (relevant) and orientation (irrelevant) with only one value (CI) on the orientation dimension $\left(15^{\circ}\right)$. 
and no interaction $(F<1)$.

\section{Experimental groups.}

Comparisons between the eight experimental groups can be made to examine:

1) the effect of the irrelevant dimension in the Phase-1 training array (Groups CI-2D versus 2D-2D)

2) the effect of the irrelevant dimension in the Phase-2 transfer array (Groups 2D-CI versus 2D-2D)

3) the effect of holding an irrelevant dimension constant in both Phase-1 and Phase-2 (Group CI-CI versus 2D-2D).

\section{1) Constant-irrelevant versus two-dimensional in Phase-1.}

In order to determine the influence of the irrelevant dimension during Phase-1 training on stimulus control established in Phase-2, gradients for Groups CI-2D and Groups 2D-2D (Figures 18 and 19) were compared. Only Phase-1 training differed between these groups. Individual (Figure 19), and group mean (Figure 18) generalisation gradients and within-group dimensional effects (Table 13) for these four experimental groups, show that holding length constant in Phase-1 leads to better control by orientation in Phase-2. There is also significantly less residual control by length in Phase-2.

Transfer data for the four groups CI-2D (12), CI-2D (20), $2 \mathrm{D}-2 \mathrm{D}(12)$ and $2 \mathrm{D}-2 \mathrm{D}(20)$ were submitted to a three-way analysis of variance with repeated measures on the factor of orientation 
113.
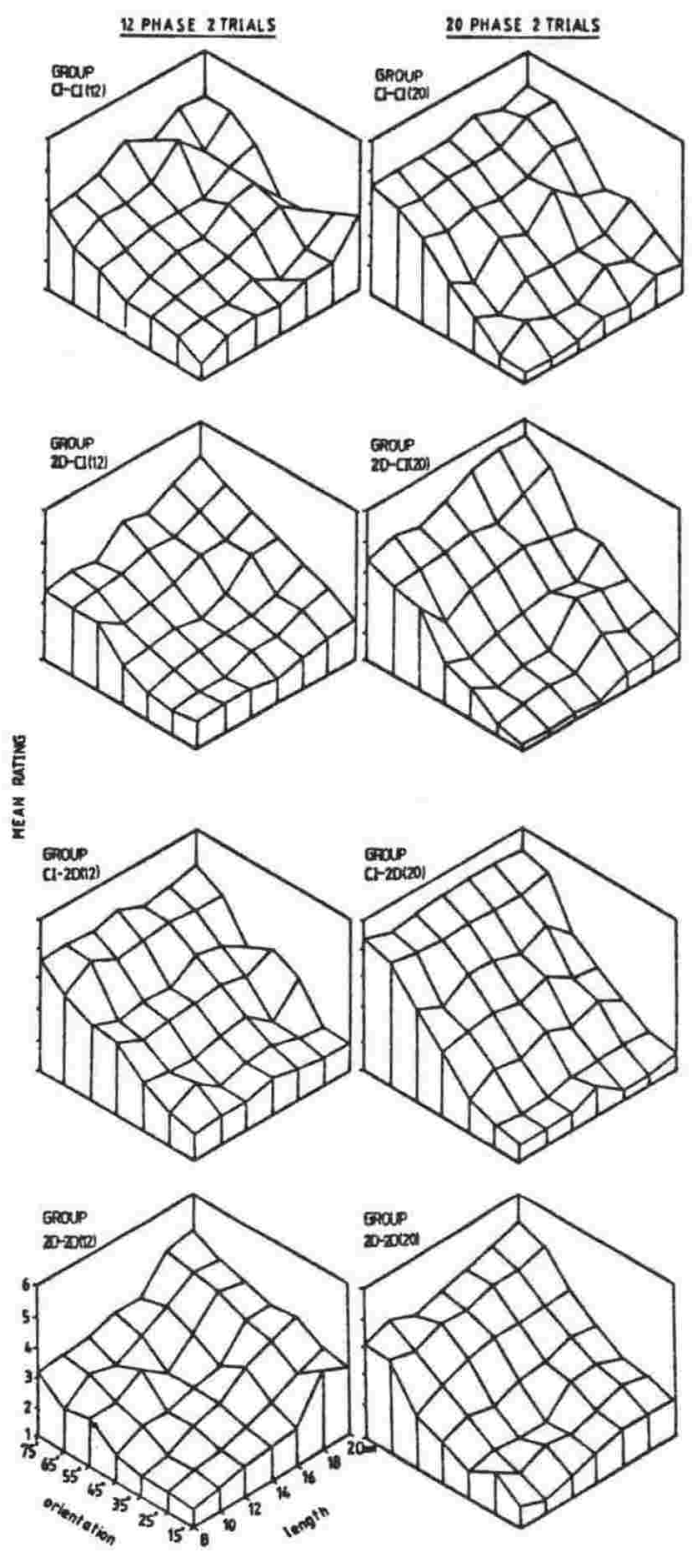

Figure 18: Post-Phase-2 two-dimensional generalisation gradients showing mean response ratings as a function of length and orientation dimensions following Phase-2 ED discrimination training with orientation (relevant) and length (Irrelevant). In Phase-1 and Phase-2 there were either two values (2D) or one value (CI) on the irrelevant dimension. 

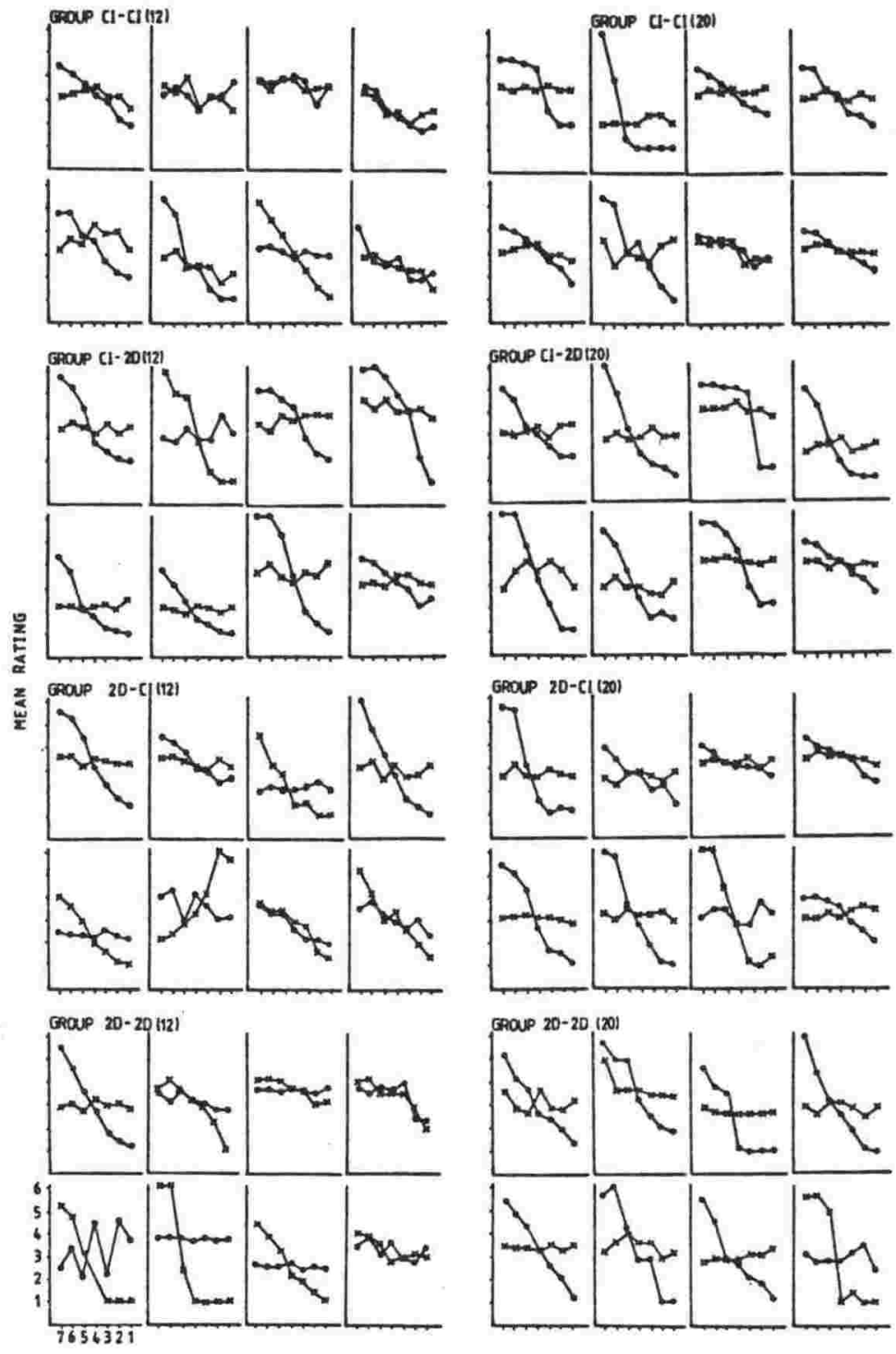

OROINAL STLHULUS VALUE ON LENGTH(*) OR OFUENTATION (*) OHMENSIONS

Figure 19: Generalisation gradients showing mean response ratings for individual subjects as a function of length $(x)$ and orientation ( $\bullet$ following Phase-2 ED training with orientation (relevant) and length (irrelevant). In Phase-1 and Phase-2 there were either two values (2D) or one value (CI) on the irrelevant dimension. 
115.

TABLE 13

F-values for two-way analyses of variance on generalisation gradients for each of the eight conditions in Experiment 5.

$\begin{array}{lll}\text { Group } & \begin{array}{l}\text { Length } \\ \text { Dimension }\end{array} & \begin{array}{l}\text { Orientation } \\ \text { Dimension }\end{array}\end{array}$

\begin{tabular}{llrl}
\hline CI - CI (12) & $4.08^{\star \star}$ & $10.18^{\star \star \star}$ & 1.06 \\
CI - CI (20) & 1.10 & $19.05^{\star \star \star}$ & 1.09 \\
$C I-2 D(12)$ & 0.85 & $16.34^{\star \star \star}$ & 1.28 \\
CI - 2D (20) & 1.13 & $39.52^{\star \star \star}$ & 0.97 \\
$2 D-C I(12)$ & 1.53 & $7.80^{\star \star \star}$ & 0.58 \\
$2 D-C I(20)$ & 0.96 & $12.53^{\star \star \star}$ & 0.91 \\
$2 D-2 D(12)$ & $5.86^{\star \star}$ & $3.56^{\star \star}$ & 0.64 \\
$2 D-2 D(20)$ & 0.81 & $16.80^{\star \star \star}$ & 0.96 \\
\hline
\end{tabular}

$$
\begin{aligned}
\star & p<.05 \\
\star \star & p<.01 \\
\star \star \star & p<.001
\end{aligned}
$$


(Winer, 1962, p.338). Results are summarised in Table 14. Two-way interactions between Array and Dimension and Trials and Dimension, but no significant three-way interaction, made it necessary to inspect the data further. Separate two-way analyses of variance (Keppel, 1982, p.438) were undertaken to compare Groups CI-2D (12) versus 2D-2D (12) and Group CI-2D (20) versus 2D-2D (20) (refer Appendix 1, Table 4). A Trial x Dimension interaction for CI-2D and 2D-2D 12 trials and not for CI-2D and 2D-2D 20 trials confirms that the effect of the array is significant after 12 trials, but attenuated by 20 trials of Phase-2 discrimination learning.

When transfer data for these four experimental groups are expressed in terms of correct number of responses over transfer trials, the same kind of picture emerges. These results for Groups CI-2D (12) and CI-2D (20) (Figure 20) show a relatively rapid transfer from random responding on the first few trials to near perfect performance by trials 8 and 9 , and stabilisation at that level for all further trials. In contrast, the same graphs for Groups 2D-2D (12) and 2D-2D (20) (Figure 20) show a slower transfer to the Phase-2 task. Generalisation gradients for these groups can therefore be seen to reflect the rate of change described in the transfer graphs for these four groups.

In summary, this comparison shows that having the relevant dimension of Phase-2 previously irrelevant in Phase-1 training retards the acquisition of the new discrimination relative to the case where the dimension is constant-irrelevant in Phase-1. 
TABLE 14

Summary of three-way analysis of variance to show between group effects of Type of Phase-1 Stimulus Array and Number of Trials as a function of Dimension in Phase-2 ED discrimination training for Groups CI-2D (12) and (20), and Groups 2D-2D (12) and (20).

\begin{tabular}{lcccc}
\hline Source & DF & MS & F & \\
\hline ARRAY (CI-2D vS 2D-2D) & 1,28 & 2.3 & $<1$ & $\mathrm{n} . \mathrm{s}$. \\
TRIALS (12 or 20) & 1,28 & 0.01 & $<1$ & $\mathrm{n} . \mathrm{s}$. \\
ARRAY x TRIALS & 1,28 & 0.9 & $<1$ & $\mathrm{n} . \mathrm{s}$. \\
DIMENSION (ORIENTATION) & 6,168 & 40.9 & 72.9 & $\mathrm{p}<.001$ \\
ARRAY x DIMENSION & 6,168 & 1.4 & 2.3 & $\mathrm{p}<.001$ \\
TRIALS x DIMENSION & 6,168 & 2.3 & 4.2 & $\mathrm{p}<.001$ \\
ARRAY X TRIALS X & 6,168 & 0.6 & $<1$ & $\mathrm{n} . \mathrm{s}$. \\
DIMENSION & & & &
\end{tabular}


12 TRIALS
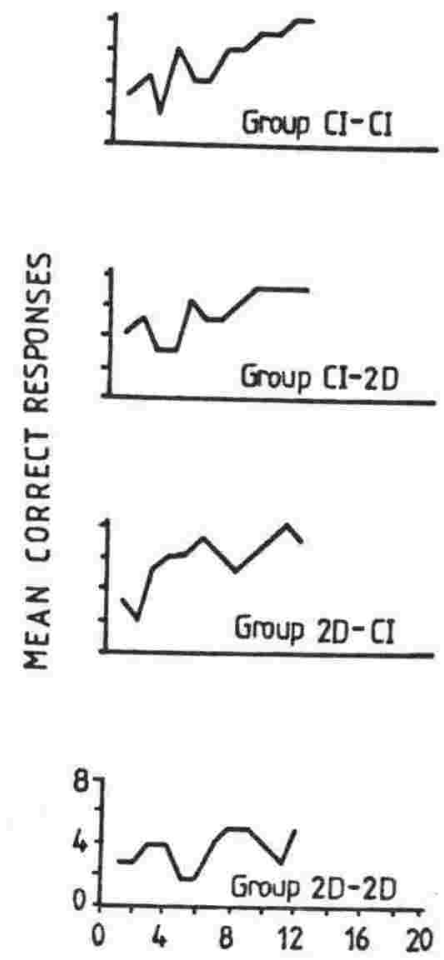

20 TRIALS
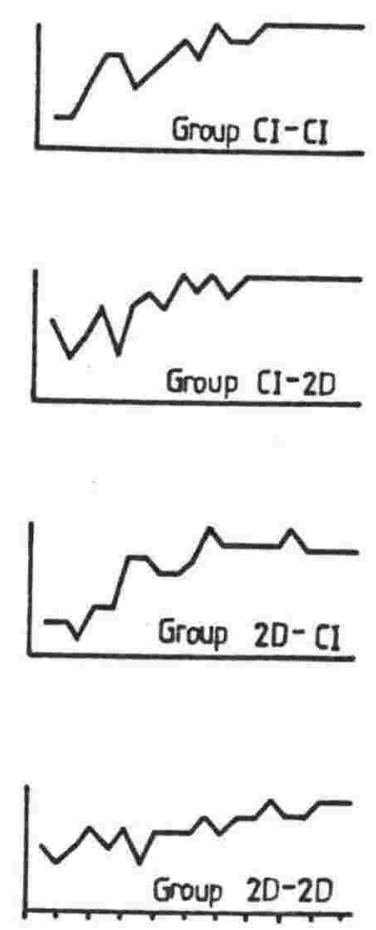

TRIALS

Figure 20: Number of correct responses per group as a function of the number of trials of Phase-2 ED training following four combinations of Phase-1 and Phase-2 discrimination training with either two (2D) or one (CI) value on the irrelevant dimension. 
2) Constant-irrelevant versus two-dimensional in Phase-2.

In order to examine the effect of the irrelevant dimension in Phase-2, gradients for Groups $2 \mathrm{D}-\mathrm{CI}$ and $2 \mathrm{D}-2 \mathrm{D}$ (Figures 18 and 19) were compared. Only Phase-2 differed between these groups. The differences in changes following ED transfer for Groups 2D-2D and 2D-CI are not nearly as marked as for the earlier comparison where the training procedure was varied.

A three-way analysis of variance with repeated measures on the factor of Dimension (Winer, 1962, p.338) was conducted to compare Array, Trials and Dimension (orientation). Main effects of Array and Trials (Table 15) were not significant, but Dimension was significant. The only significant interaction for these groups was Trials $\times$ Dimension. When the data for these two conditions was collapsed over trials (Keppel, 1982, p.438) no differences were revealed at either 12 or 20 trials for Array $\times$ Dimension effects (refer Appendix I, Table 5).

The transfer data (Figure 20) illustrate how these changes are taking place over trials. Both transfer Groups 2D-CI (12) and (20) appear to show a faster rate of change from random responding over the first few trials to a more consistent performance after trial 12, compared to the performance after the same number of trials in both $2 \mathrm{D}-2 \mathrm{D}(12)$ and (20). However, this difference has been shown not to be a significant one.

In summary, although transfer to the CI array does appear to 


\section{TABLE 15}

Summary of three-way analysis of variance to show between group effects of Type of Phase-2 Stimulus Array and Number of Trials as a function of Dimension in Phase-2 ED discrimination training For Groups 2D-CI (12) and (20), and Groups 2D-2D (12) and (20).

\begin{tabular}{|c|c|c|c|c|}
\hline Source & DF & MS & $\mathrm{F}$ & \\
\hline ARRAY (2D-CI vs 2D-2D) & 1,28 & 0.1 & $<1$ & n.s. \\
\hline TRIALS (12 or 20) & 1,28 & 0.02 & $<1$ & n.s. \\
\hline ARRAY $\times$ TRIALS & 1,28 & 1.1 & $<1$ & n.s. \\
\hline DIMENSION (ORIENTATION) & 6,168 & 25.8 & 44.5 & $p<.001$ \\
\hline ARRAY $x$ DIMENSION & 6,168 & 0.1 & $<1$ & n.s. \\
\hline TRIALS $\times$ DIMENSION & 6,168 & 2.5 & 4.3 & $p<.001$ \\
\hline $\begin{array}{l}\text { ARRAY } x \text { TRIALS } \times \\
\text { DIMENSION }\end{array}$ & 6,168 & 0.5 & $<1$ & n.s. \\
\hline
\end{tabular}


121.

have a small advantage in terms of rate of transfer to the Phase-2 discrimination, having the irrelevant dimension held constant in the Phase-2 array does not lead to the marked difference in performance that was observed between groups where the irrelevant dimension was held constant in the Phase-1 training.

\section{3) CI-CI condition.}

The CI-CI condition represents the case where the irrelevant dimension has been held constant in both Phase-1 and Phase-2 discrimination training. Compared to the other three experimental conditions (Figures 18 and 19) the generalisation gradient for Group CI-CI (12) shows that as well as the gradients for orientation being relatively steep, the gradients for the length dimension are also relatively steep. The analysis of variance (Table 13) confirms this trend. This suggests that not only has the new Phase-2 dimension, orientation, gained control after only 12 trials, but also, the Phase-1 relevant dimension still exerts control over responding. By 20 trials the Group CI-CI (20) generalisation gradient (Figure 18 (i)), is virtually the same as either the Group CI-2D (20) or the 2D-CI (20) generalisation gradient. Examining the transfer of training data, Figure 20, it can be seen that the CI-CI condition is shown to have transferred to the Phase-2 discrimination more quickly than any other group. However, as the Phase-2 irrelevant dimension, length, was not made explicitly irrelevant in Phase-2, but was held constant-irrelevant, responding to this dimension occurred again in the generalisation test and therefore control by the new relevant dimension, orientation was not as strong as might have been expected 
from the transfer of training, trial-by-trial data (Figure 20).

In order to establish the nature of control by the length dimension following CI Phase-1 training, and to compare it to the control established after 2D Phase-1 training, the control CI group was run. It could not be assumed that the order of control was the same following the two different types of training, and in view of the CI training group results, it appeared that following CI Phase-1 training, control by the length dimension could be weaker. However, as has been noted, the generalisation gradient for Group CI control (Figure 17) following 24 trials of CI Phase-1 constant-irrelevant training does not differ from the gradients (Figures 18 and 19) following 24 trials of Phase-1 two-dimensional training.

\section{DISCUSSION}

Lovejoy (1968), Sutherland and Mackintosh (1971) and Fisher and Zeaman (1973) predict that the ID-ED shift would be eliminated or attenuated if the dimension relevant in Phase- 2 was ConstantIrrelevant in Phase-1. Sutherland and Mackintosh (1971) suggest that the positive transfer would be a result of the neutralising of irrelevant stimuli during the Phase-1 discrimination training.

The first comparison of 2D-2D groups versus CI-2D groups is consistent with this prediction that ED transfer is accelerated when the irrelevant dimension is held constant in Phase-1. This would therefore reduce an ID-ED shift difference. A second comparison looked for any similar advantage that holding the irrelevant 
dimension constant in Phase-2 might have, but no significant improvement performance of 2D-CI groups over 2D-2D groups was found. It would appear, therefore, that 'learned irrelevance' in Phase-1 training had the biggest effect on the acquisition of the Phase-2 ED task.

However, an issue for theories of attention in interpreting empirical data has been raised by the CI-CI group. In previous experiments examining the ID-ED shift in adult humans (Esposito, 1975b: Shanab \& Yasin, 1979) and in the current series of experiments, subjects are invariably tested for the acquisition of the Phase-2 task in conditions where both experimental dimensions are present. Irrespective of whether the procedure involves discrete trials to criterion or generalisation testing, a common problem is evident. If the subject does not respond to a particular dimension, does this represent lack of attention to that dimension, or stronger control by the other alternative dimension? For example, in a generalisation gradient does weak stimulus control by a dimension represent no control (ie. no attention) or, represent a response strategy learned in Phases 1 and 2? In the CI-CI group, lack of correspondence between trial by trial data and post-discrimination generalisation gradients points out a potential problem in any two-dimensional testing procedure, but one that is most apparent when examining generalisation gradients.

In order to differentiate between cue utilisation during testing and cue selection during training, Experiment 5 is replicated 
in Experiment 6, with the relevant dimension of Phase-1 training, length, removed (held constant at one value) during the generalisation test. The extent of control by the Phase-2 dimension, orientation, can then be examined without the possibility of the previous Phase-1 dimension masking stimulus control in the test itself. 


\section{EXPERIMENT 6}

In order to demonstrate differential control by any one dimension Honig (1970) argues that two conditions must be met in the experimental design. First, it "is necessary to show that such selection can be brought under experimental control" (p.197). This has been established in Experiments $1-5$. Second, it must be shown that "such a selection occurs during training and is not due to the simultaneous presentation of several cues in testing, one of which may be 'dominant' and prevent other cues from demonstrating any stimulus control" (p.197). In other words we must distinguish between cue utilisation during testing and cue selection during training.

An experiment involving two-dimensional visual stimulus control in pigeons was performed by Newman and Baron (1965). Following ten days of single-stimulus VI training to respond to a compound stimulus, a white vertical line on a green background, Newman and Baron administered generalisation tests. The tests comprised five different orientations of white line, superimposed on a green background. No differential responding to the five orientations was apparent and Newman and Baron inferred from the flat gradient for orientation that colour was the dominant dimension and its presence has precluded any learning about line angle during discrimination training. The subjects had therefore attended to colour and ignored orientation. Freeman and Thomas (1967) argued that rather than this result being a function of what had been learned, it was a function of the testing procedure itself. Freeman and Thomas (1967) 
replicated Newman and Baron's (1965) training procedures but tested the birds in two ways (Figure 21). They found, using an increased range of values on the orientation dimension, that when a neutral background was used in testing, the gradient was significantly steeper with respect to orientation, than when the green training background was used. In Newman and Baron's study, the birds had therefore "attended" to the dimension during training, but the presence of the dominant dimension, colour, has masked control by orientation during testing. A similar result was found in an experiment conducted by Newman and Benefield (1968). Testing in neutral conditions produced a reliably steeper gradient of responding to orientation than testing with the previous green background used in training.

In the light of this research, Thomas et al. (1970) conducted a series of experiments which specifically examined the effects of extradimensional discrimination training on stimulus generalisation. In Experiments I - IV, reported by Thomas et al. (1970), generalisation testing was carried out along one dimension only and the other training dimension was removed from the test stimuli. Thomas et al. (1970) were attempting to distinguish between "attention (ie. cue selection during training) and cue utilisation during testing, as factors influencing the slope of generalisation gradients" (Thomas et al., 1970, p.330).

"Attention" to a particular dimension may be inferred from a sloping gradient in a generalisation test. However, it is not 


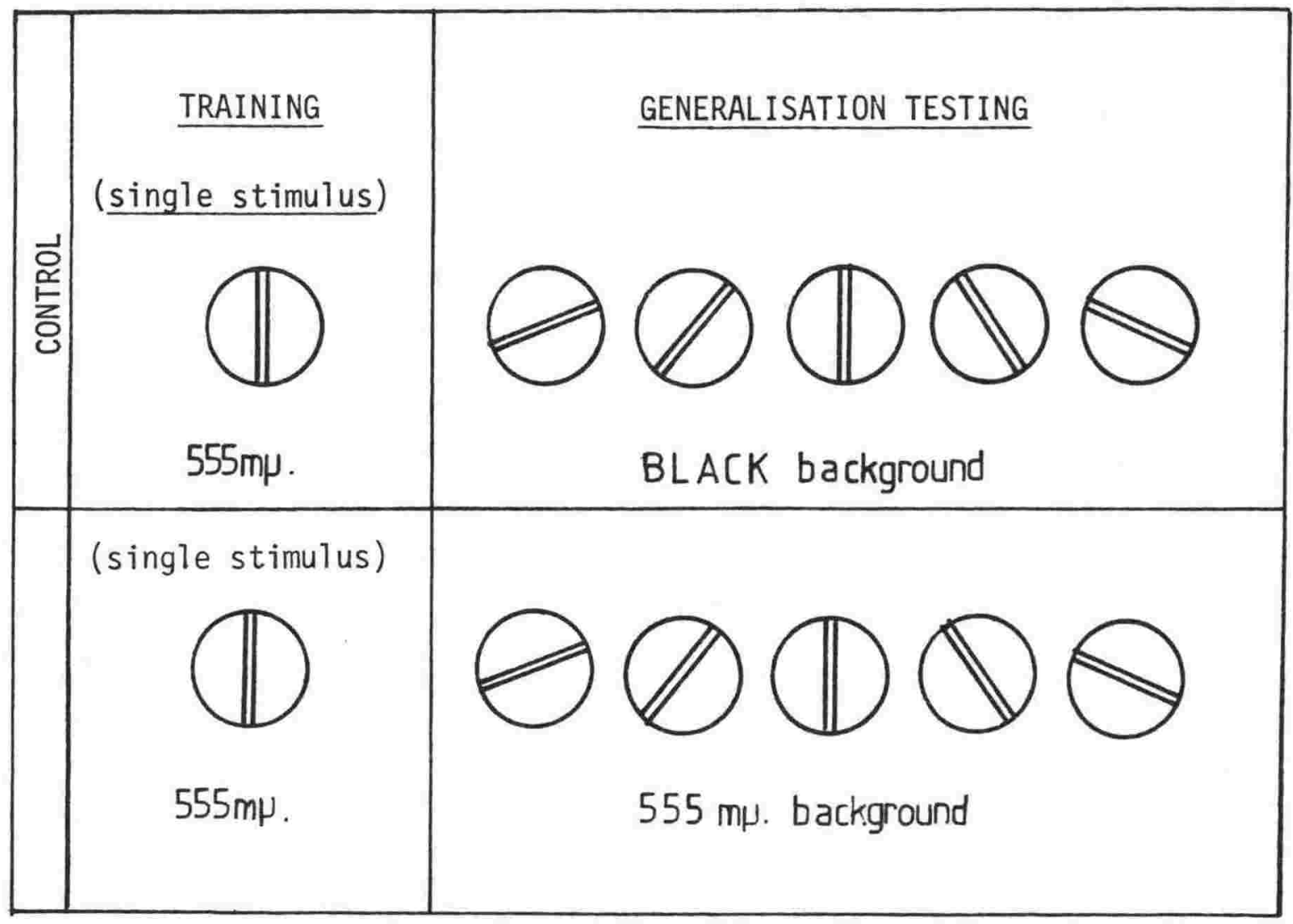

Figure 21: A schematic representation of the two testing conditions in the Freeman and Thomas (1967) experiment. 
128.

equally valid to assume that a relatively flat gradient means the subject is not attending to that dimension. Conditions of the test itself also influence the slope of generalisation gradients when under other test conditions attention to a particular dimension is clearly in evidence as has been demonstrated (Freeman \& Thomas, 1967: Newman \& Baron, 1965: Newman \& Benefield, 1968)

Honig and Urcuioli (1981) recognise masking as a testing phenomenon that can modulate attention to criterion stimuli. It is important therefore, that masking of stimulus control by a competing dimension should be considered before attention or its absence is inferred from a generalisation gradient. In general, in the ID-ED paradigm, attention to one dimension is assessed in the presence of other dimensions which may or may not have been relevant, irrespective of whether trial to criterion, errors to criterion or generalisation tests have been administered. There have been few exceptions in the human field (Hogg \& Evans, 1975).

In the present experiment, Experiment 5 was replicated, but instead of varying the orientation and length dimensions simultaneously in the generalisation test, only one dimension, the dimension of acquisition, orientation, was varied and the length was held constant at a single value.

METHOD

Subjects were 80 undergraduates. Equal numbers of males and females were randomly assigned to one of two control and eight 
experimental groups. The eight experimental groups were identical to those used in Experiment 5. The apparatus used and the general procedural conditions were the same as those in Experiment 5, with the exception of the stimuli used in the generalisation test.

Phase-1 discrimination training. Phase-1 discrimination training was the same as in the experimental groups in Experiment 5 . An additional 16 subjects were run in two control groups. These subjects were given Phase-1 two-dimensional or constant-irrelevant training only followed immediately by a one-dimensional generalisation test.

Phase-2 discrimination training. The same Phase-2 discrimination training was administered as in Experiment 5.

Generalisation testing. A generalisation test was administered directly following the pre-determined number of Phase-2 discrimination trials without a break in the procedure. The test comprised presentations of 49 stimuli combining seven orientation values with a constant $(20 \mathrm{~mm})$ value of the length dimension in seven different random orders. The random order of presentation over the first 49 trials was reversed for a second block of 49 trials.

\section{RESULTS AND DISCUSSION}

\section{Control groups}

The data for the two groups of eight subjects run in a control 
group for the generalisation test following Phase-1 constantirrelevant training and Phase-1 two-dimensional training are shown in Figure 22( $i)$ and (ii) respectively. It can be seen that following 24 trials of Phase-1 training on the length dimension, there is no control by the dimension of orientation for either group when orientation is varied alone in the generalisation test. An analysis of variance supported this finding with no effect of orientation ( $F \quad 1$ ) in either condition. This compares with the control groups in Experiments 1 (Figure 5) and 5 (Figure 17) where orientation has no dimensional stimulus control.

\section{Experimental groups}

Comparisons between the eight experimental groups are the same as those in Experiment 5 .

1) The mean data plotted in Figure 23 parts (ii) and (iv) show that subjects in Group CI-2D (12) exhibit more rapid transfer to the twodimensional ED problem than Group 2D-2D (12). After a further eight trials in the condition CI-2D (Figure 23 (ii)), the control by the orientation dimension has shown no marked change, particularly when the individual data (Figure 24 (ii)) for Group CI-2D (12) and Group CI-2D (20) is compared. Control by orientation in the 2D-2D condition, however, is markedly stronger in Group 2D-2D (20) than in the same group after 12 trials (Figure 23 (iv)). The differences between Group CI-2D and Group 2D-2D are therefore attenuated by 20 trials. A three-way analysis of variance with repeated measures on the factor of orientation (Winer, 1962, p.338) is summarised in 


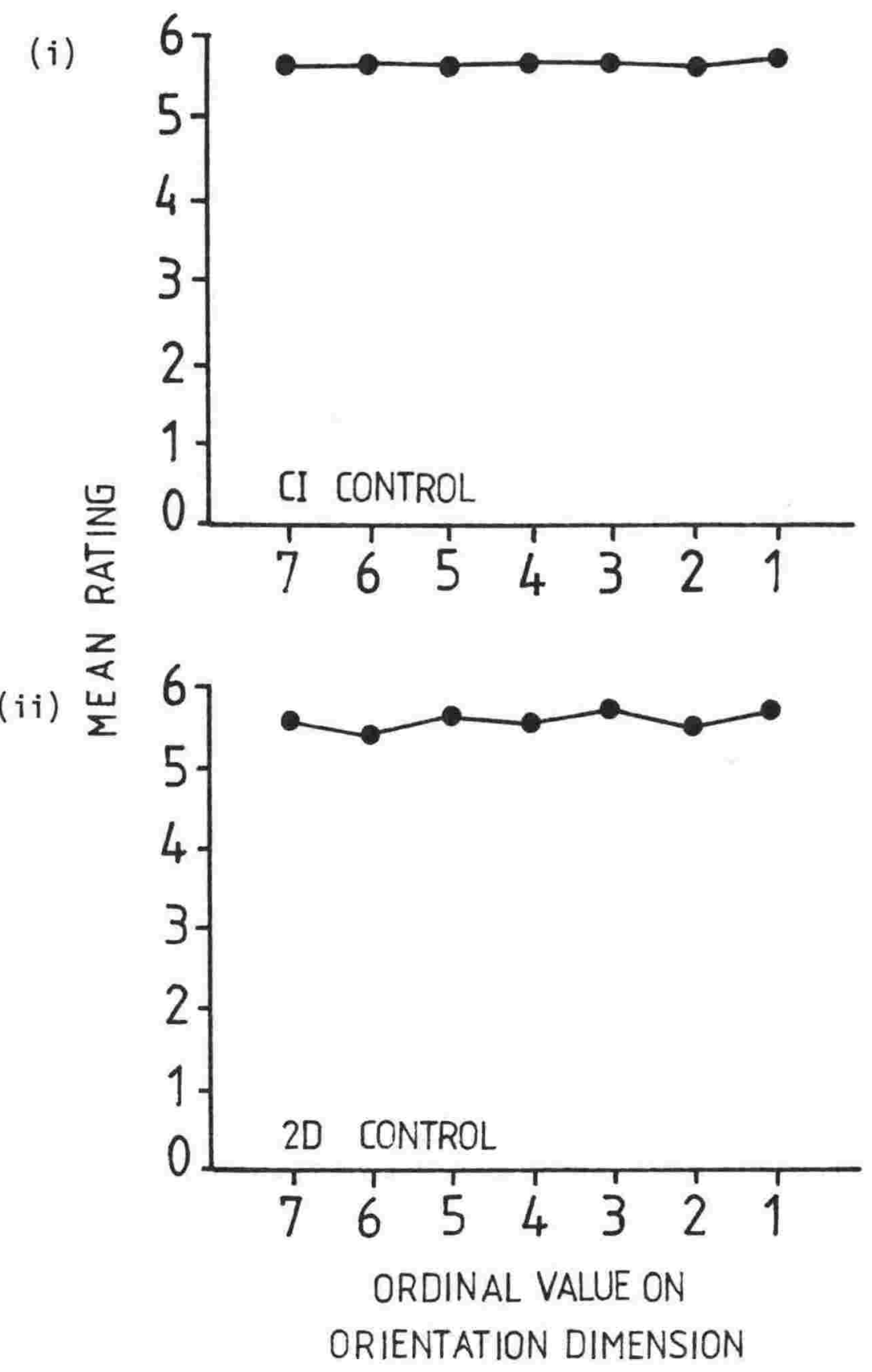

Figure 22: Generalisation gradients along the dimension of orientation (o) following 24 trials of Phase-1 training with either (i) length (relevant) and orientation (constant-irrelevant:CI) or ( $i i$ ) length (relevant) and orientation (irrelevant:2D). 
132.

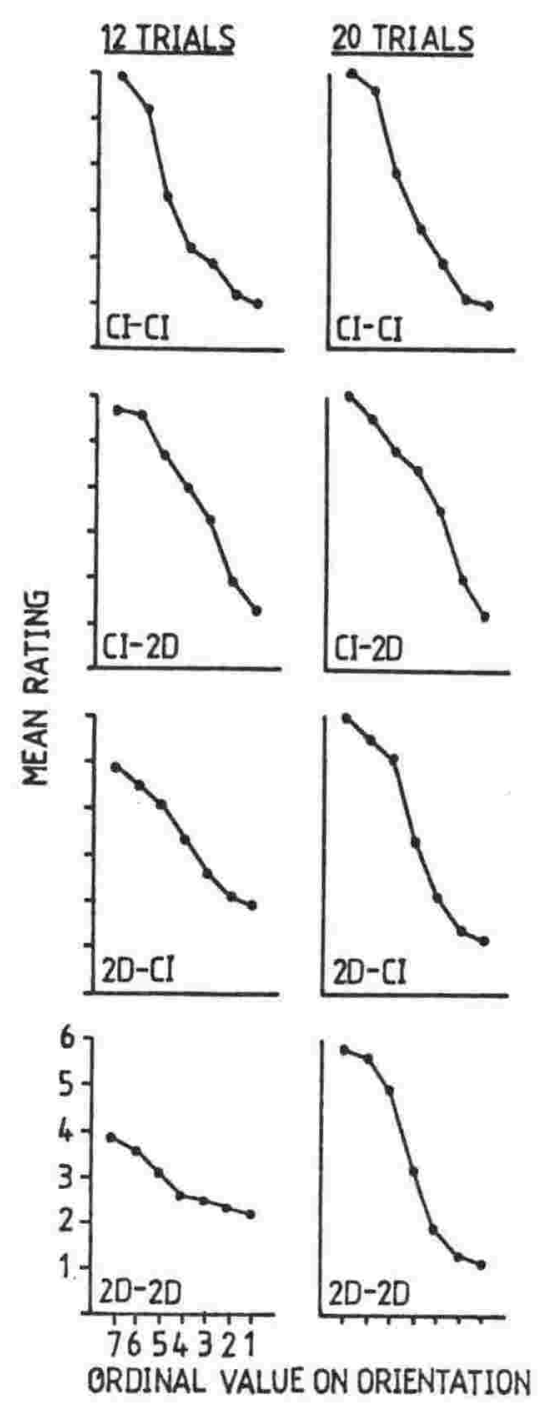

Figure 23: One-dimensional generalisation gradients showing group mean response ratings as a function of orientation ( $(\bullet)$ following Phase-2 ED training with orientation (relevant) and length (irrelevant). In Phase-1 and Phase-2 there were either two values (2D) or one value (CI) on the irrelevant dimension. 
12 PHASE 2 TRIALS
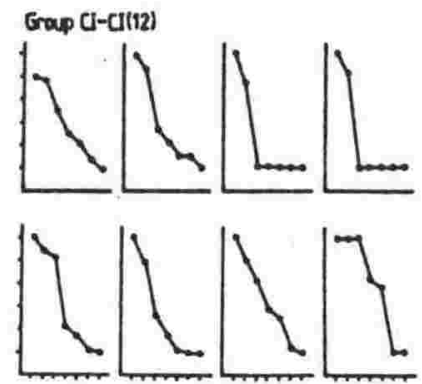

Grap [-20112]
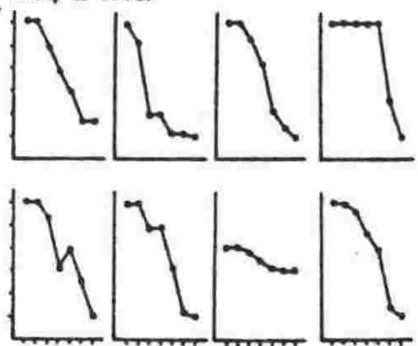

Group 20-C112)
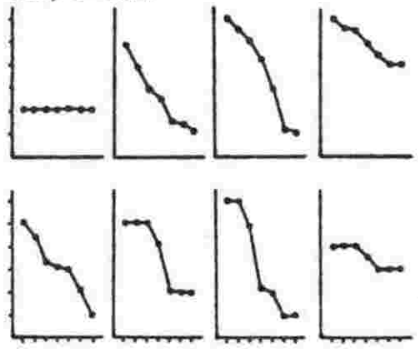

Group 2D-2D(12)
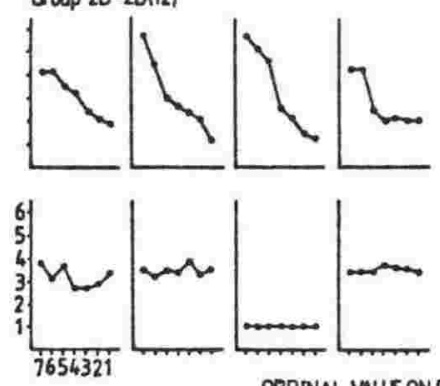

20 PHASE 2 TRIALS

Group c-a(20)
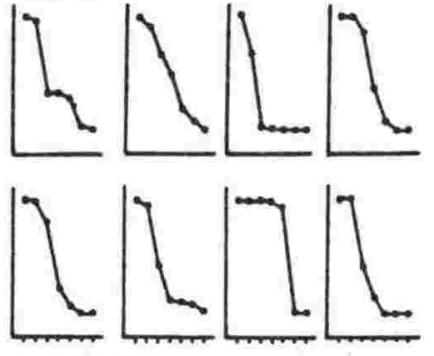

Group Q-201201
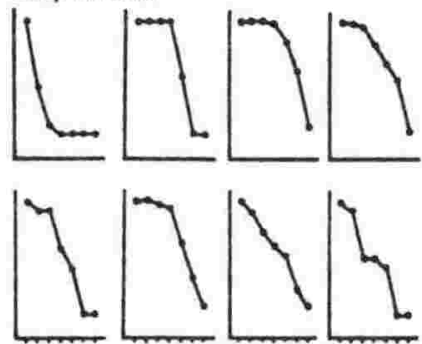

Group 20-C1(20)
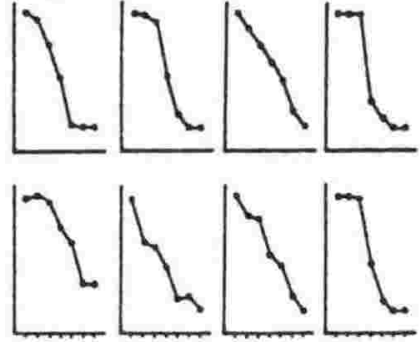

Group 20-20(20)
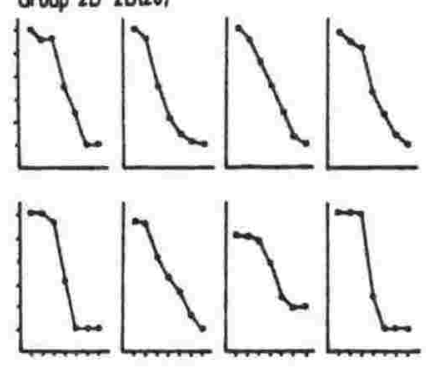

Figure 24: One-dimensional generalisation gradients showing mean response ratings for individual subjects as a function of orientation ( ) following Phase-2 ED training with orientation (relevant) and length (irrelevant). In Phase-1 and Phase-2 there were either two values (2D) or one value (CI) on the irrelevant dimension. 
Table 16, and together with separate two-way analyses (Appendix 1, Table 6) confirms this finding.

When transfer data for these four experimental groups are expressed in terms of correct number of responses over transfer trials, the same kind of pattern emerges. These results for Groups CI-2D (12) and (20) (Figure 25) show a relatively rapid transfer from random responding on the first few trials to near perfect performance by trial 12, stabilising at that level for all further trials. In contrast, the same graphs for Groups 2D-2D (12) and (20) show a slower transition to the Phase-2 task. Generalisation gradients for these groups can therefore be seen to reflect the rate of change described in the training data for these four groups.

In summary, these results show that holding the irrelevant dimension constant in Phase-1 training enhances control by the new relevant dimension in Phase-2 and that this result obtains irrespective of whether the relevant dimension of Phase-1 varies throughout the generalisation test (seven values) as in Experiment 5 or remains constant (one value) as in Experiment 6.

\section{2) Constant-irrelevant versus two-dimensional in Phase-2}

There do not appear to be any marked differences following the two types of ED transfer for Groups 2D-CI (12) and 2D-2D (12) (Figures 23 and 24). After a further eight trials the performance for both groups indicate the same order of control by orientation for Groups 2D-CI (20) and 2D-2D (20), Figure 23 (iii) and (iv). A 


\section{$\underline{\text { TABLE } 16}$}

Summary of three-way analysis of variance to show between group effects of Type of Phase-1 Stimulus Array and Number of Trials as a function of Dimension in Phase-2 ED discrimination training for Groups CI-2D (12) and (20), and Groups 2D-2D (12) and (20).

\begin{tabular}{lcccc} 
Source & DF & MS & $F$ & \\
\hline ARRAY (CI-2D vS 2D-2D) & 1,28 & 20.9 & 5.7 & $\mathrm{p}<.05$ \\
TRIALS (12 or 20) & 1,28 & 4.6 & 1.3 & $\mathrm{n} . \mathrm{s}$. \\
ARRAY x TRIALS & 1,28 & 1.8 & $<1$ & $\mathrm{n} . \mathrm{s}$. \\
DIMENSION (ORIENTATION) & 6,168 & 76.9 & 148.0 & $\mathrm{p}<.001$ \\
ARRAY X DIMENSION & 6,168 & 2.6 & 5.0 & $\mathrm{p}<.001$ \\
TRIALS X DIMENSION & 6,168 & 4.3 & 8.3 & $\mathrm{p}<.001$ \\
$\begin{array}{l}\text { ARRAY X TRIALS X } \\
\text { DIMENSION }\end{array}$ & 6,168 & 4.5 & 8.6 & $\mathrm{p}<.001$
\end{tabular}


12 TRIALS
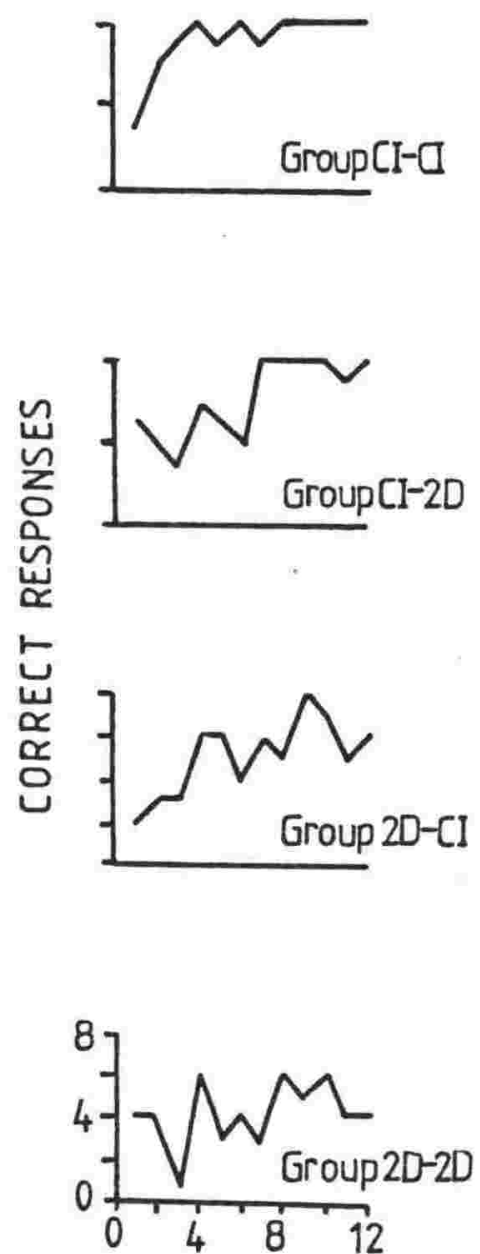

20 TRIALS
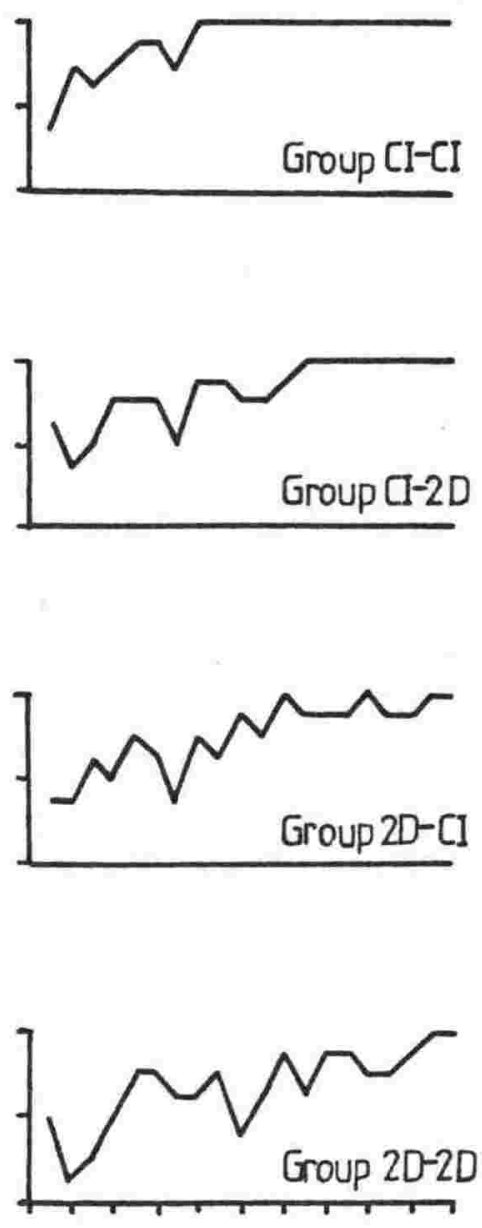

TRIALS

Figure 25: Number of correct responses per group as a function of the number of trials of Phase-2 ED training following four combinations of Phase-1 and Phase-2 discrimination training with either two (2D) or one (CI) values on the irrelevant dimension. 
three-way analysis of variance with repeated measures on the factor of orientation (Winer, 1962, p.338) is summarised in Table 17. No main effect was significant. The only significant interaction for these groups was Trials $\times$ Dimension, which indicated a different order of control by orientation after 12 or 20 trials. When data for the two conditions were collapsed over trials, the two-way analyses of variance (refer Appendix I, Table 7) revealed no differences at either 12 or 20 trials for Array x Dimension effects.

The transfer data (Figure 25) illustrate how these changes are taking place over trials. Groups 2D-CI (12) and 2D-2D (12) show no marked difference in rate of acquisition of the task. The equivalent groups after 20 trials also show no marked difference in rate.

In summary, the analyses and trials of transfer data do not reveal any superior performance by the $2 D-C I$ condition with respect to dimensional stimulus control by orientation in either Experiment 5 or Experiment 6.

\section{3) Constant-irrelevant to constant-irrelevant}

In Experiment 5 the results from this group proved to be ambiguous. While the trials of transfer graph (Figure 20) showed relatively fast transfer by the CI-CI groups, the generalisation gradients (Figures 18 and 19) showed poor control by orientation after 12 trials of Phase-2 training, although control by this dimension has improved by 20 trials. It was argued that while 
138.

\section{TABLE 17}

Summary of three-way analysis of variance to show between group effects of Type of Phase-2 Stimulus Array and Number of Trials as a function of Dimension in Phase-2 ED discrimination training for Groups 2D-CI (12) and (20), and Groups 2D-2D (12) and (20).

\begin{tabular}{lcrrc}
\hline Source & DF & MS & F & \\
\hline ARRAY (2D-CI vS 2D-2D) & 1,28 & 4.0 & 1.4 & $\mathrm{n} . \mathrm{s}$. \\
TRIALS (12 or 20) & 1,28 & 6.5 & 2.3 & $\mathrm{n} . \mathrm{s}$. \\
ARRAY X TRIALS & 1,28 & 1.8 & $<1$ & $\mathrm{n} . \mathrm{s}$. \\
DIMENSION (ORIENTATION) & 6,168 & 69.4 & 144.5 & $\mathrm{p}<.001$ \\
ARRAY X DIMENSION & 6,168 & 0.7 & 1.5 & $\mathrm{n} . \mathrm{s}$. \\
TRIALS X DIMENSION & 6,168 & 10.1 & 21.1 & $\mathrm{p}<.001$ \\
ARRAY X TRIALS X & 6,168 & 0.6 & 1.3 & $\mathrm{n} . \mathrm{s}$. \\
DIMENSION & & & &
\end{tabular}


subjects in Group CI-CI learned that orientation was relevant in Phase-2, they may not have learned that length was irrelevant. Thus when presented with both dimensions in the generalisation test, control by orientation persisted from Phase-2, but in addition, residual control by length was carried over from Phase-1. This result was reflected in both the analysis of variance for this group (Table 13) where $F(6,42)=4.08 \mathrm{p}<, 01$ for length and $F(6,42)=$ $10.18 \mathrm{p}<.001$ for orientation, and in the individual gradients (Figure 19). The subjects in CI-CI were therefore in the same type of situation as Newman and Baron's (1965) pigeons.

The results of Experiment 6 showed a different order of control by the orientation dimension when the length dimension was removed in testing. Training data (Figure 25) showed the same rapid acquisition of the task as in Experiment 5, but the generalisation gradients (Figures 23 and 24) exhibited much stronger control by the CI-CI (12) group when tested only on the orientation dimension. The strong control by orientation was true for all subjects. In contrast to the same gradients in Experiment 5, the CI-CI groups in Experiment 6 show the strongest control by the orientation dimension of any group. This difference between Experiments 5 and 6 prompted a further comparison of the data from the two experiments.

\section{Comparison of Experiments 5 and 6}

Equivalent conditions in Experiments 5 and 6 involved identical Phase-1 and Phase-2 training. The only difference in the procedure was that, in Experiment 5, both orientation and length were varied 


\section{TABLE 18}

Summary of analyses of variance comparing effects of one and two dimensional generalisation tests on control by the orientation dimension in the ED shift.

\begin{tabular}{|c|c|c|c|c|c|}
\hline Group & Source & $\mathrm{DF}$ & MS & $\mathrm{F}$ & \\
\hline $\mathrm{CI}-\mathrm{CI}$ (12) & $\begin{array}{l}\text { Test } \\
\text { Dimension } \\
\text { Test x Dim }\end{array}$ & $\begin{array}{l}1,14 \\
6,84 \\
6,84\end{array}$ & $\begin{array}{r}0.2 \\
26.9 \\
6.4\end{array}$ & $\begin{array}{c}<1 \\
55.3 \\
13.2\end{array}$ & $\begin{aligned} & n . s \\
p & <.001 \\
p & <.001\end{aligned}$ \\
\hline CI-CI (20) & $\begin{array}{l}\text { Test } \\
\text { Dimension } \\
\text { Test x Dim }\end{array}$ & $\begin{array}{l}1,14 \\
6,84 \\
6,84\end{array}$ & $\begin{array}{c}0 \\
26.9 \\
4.4\end{array}$ & $\begin{array}{r}<1 \\
60.8 \\
7.2\end{array}$ & $\begin{array}{l}\quad n . s . \\
p<.001 \\
p<.01\end{array}$ \\
\hline$C I-2 D(12)$ & $\begin{array}{l}\text { Test } \\
\text { Dimension } \\
\text { Test x Dim }\end{array}$ & $\begin{array}{l}1,14 \\
6,84 \\
6,84\end{array}$ & $\begin{array}{r}12.8 \\
38.9 \\
2.6\end{array}$ & $\begin{array}{r}4.0 \\
67.8 \\
4.6\end{array}$ & $\begin{array}{l}\quad n . s . \\
p<.001 \\
p<.01\end{array}$ \\
\hline$C I-2 D \quad(20)$ & $\begin{array}{l}\text { Test } \\
\text { Dimension } \\
\text { Test x Dim }\end{array}$ & $\begin{array}{l}1,14 \\
6,84 \\
6,84\end{array}$ & $\begin{array}{r}10.3 \\
41.8 \\
1.2\end{array}$ & $\begin{array}{r}2.6 \\
77.7 \\
2.3\end{array}$ & $\begin{aligned} & n .5 \\
p & <.001 \\
p & <.05\end{aligned}$ \\
\hline 2D-CI (12) & $\begin{array}{l}\text { Test } \\
\text { Dimension } \\
\text { Test x Dim }\end{array}$ & $\begin{array}{l}1,14 \\
6,84 \\
6,84\end{array}$ & $\begin{array}{r}4.9 \\
14.2 \\
1.1\end{array}$ & $\begin{array}{r}1.4 \\
23.2 \\
1.7\end{array}$ & $p<\begin{array}{r}n . s . \\
\\
n . s .001 \\
n .\end{array}$ \\
\hline $2 D-C I \quad(20)$ & $\begin{array}{l}\text { Test } \\
\text { Dimension } \\
\text { Test x Dim }\end{array}$ & $\begin{array}{l}1,14 \\
6,84 \\
6,84\end{array}$ & $\begin{array}{r}5.1 \\
37.1 \\
4.4\end{array}$ & $\begin{array}{r}6.2 \\
72.9 \\
8.7\end{array}$ & $\begin{array}{l}p<.05 \\
p<.001 \\
p<.001\end{array}$ \\
\hline 2D-2D (12) & $\begin{array}{l}\text { Test } \\
\text { Dimension } \\
\text { Test x Dim }\end{array}$ & $\begin{array}{l}1,14 \\
6,84 \\
6,84\end{array}$ & $\begin{array}{l}1.1 \\
5.8 \\
0.1\end{array}$ & $\begin{array}{l}<1 \\
9.1 \\
<1\end{array}$ & $\begin{array}{c}n . s . \\
<.05 \\
n . s .\end{array}$ \\
\hline $2 D-2 D(20)$ & $\begin{array}{l}\text { Test } \\
\text { Dimension } \\
\text { Test x Dim }\end{array}$ & $\begin{array}{l}1,14 \\
6.84 \\
6,84\end{array}$ & $\begin{array}{r}5.3 \\
44.5 \\
2.5\end{array}$ & $\begin{array}{r}5.0 \\
117.9 \\
6.6\end{array}$ & $\begin{array}{l}p<.05 \\
p<.001 \\
p<.01\end{array}$ \\
\hline
\end{tabular}


in the generalisation test, whereas in Experiment 6 only orientation was varied and length was held at a constant value. It was thus possible to test whether control by the Phase-2 relevant dimension (orientation) was attenuated by the presence of the Phase-1 relevant dimension (length) during two-dimensional generalisation tests.

Separate two-way analyses of variance (Keppel, 1982, p.438) were conducted for each pair of equivalent conditions to compare control by the orientation dimension for the two testing situations. A summary of these analyses can be found in Table 18. Comparing Figures 19 and 24 and Table 18, two main findings are evident. Firstly, when stimulus control by orientation was strong in Experiment 5, holding length constant in a generalisation test in Experiment 6 resulted in enhanced control by orientation. This effect can be observed in Groups CI-2D (12), CI-CI (20), CI-2D (20), $2 D-C I(20)$ and $2 D-2 D(20)$. The presence of length in the generalisation test therefore masks control by orientation in cases where control by orientation is well developed.

Secondly, when stimulus control by orientation was relatively poor in Experiment 5, holding length constant in the generalisation test in Experiment 6 made no difference to control by orientation. This effect can be observed in Groups 2D-CI (12) and 2D-2D (12). That is, control by orientation had not developed sufficiently to be masked by the length dimension. There appears, therefore, to be an interaction between the extent of masking by length and the extent of original control by orientation. 
GENERAL DISCUSSION 


\section{GENERAL DISCUSSION}

Wolff's (1967) summary of findings for adult students and the results of more recent studies (Nehrke, 1973: Shanab \& Yasin, 1979) generally show that in simultaneous discriminations, the ID shift is performed faster or more accurately than the ED shift over a range of conditions. The present experiments examined the ID-ED shift in successive two-dimensional discriminations using data from post-discrimination generalisation tests to determine the nature of stimulus control in the ID-ED shift.

In Experiment 1 the ID-ED difference was evident both when data were expressed in terms of speed of acquisition (cf. 0zioko \& May, 1977: Shanab \& Yasin, 1979), and in terms of stimulus control. It was found that the relationship between the trials to criterion data and the degree of control by the two dimensions at criterion was a consistent one. The post-discrimination generalisation gradients at criterion showed strong control by the Phase-2 relevant dimension and weak control by the irrelevant dimension of Phase-2.

Previous studies have identified two factors in adult discrimination learning that can potentially affect the extent of the ID-ED difference: the relative salience of the dimensions (Esposito, 1973: Harrow, 1964: Jzioko \& May, 1977), and the variation of the irrelevant dimension in Phase-1 (Esposito, 1975a: Ozioko \& May, 1977). In Experiment 1 the difference between ID-ED shift data when represented as both trials to criterion and generalisation gradients was of the same order for both the orientation-to-length and length- 
to-orientation groups. Therefore there was no evidence to suggest that a dominance relation existed with line length and orientation dimensions (cf. Lovejoy, 1968: Newman \& Baron, 1965: Trabasso \& Bower, 1968). It could therefore be assumed that the ID-ED shift differences were not attributable to such a factor.

Experiments 2 and $2 A$ (Appendix II) were designed to examine the possibility that dimensional control in the ID shift was mediated by cue-specific transfer because the discrimination between $20 \mathrm{~mm}$ versus $8 \mathrm{~mm}$ in Phase-1 was transferred to a discrimination between $18 \mathrm{~mm}$ versus $10 \mathrm{~mm}$ in Phase-2. It was demonstrated that the significantly faster transfer by the ID group compared to the ED group was not attributable to stimulus generalisation from Phase-1 to Phase-2 stimuli alone. Instead, the superiority of the ID condition over the ED condition provided evidence for attention to the dimensions of the stimuli.

Experiment 3 was designed to examine the development of stimulus control over the course of the Phase-2 ID or ED discrimination task (Heinemann \& Avin, 1973: Ray, 1972). Results confirmed that subjects transferred more quickly in the ID shift than in the ED shift. Post discrimination generalisation gradients showed that in an ED shift, attention to the irrelevant dimension in Phase-2 (Phase-1 relevant dimension) weakened over trials, while attention to the Phase-2 relevant (Phase-1 irrelevant) dimension increased over trials. Thus two effects are involved in the ID-ED shift difference: weakening of control by the previously relevant dimension 
144.

and strengthening of control by the new relevant dimension.

Lovejoy (1968) and Sutherland and Mackintosh (1971) argue that the theoretical postulates of acquisition and extinction implicated in learning are applied to dimension-specific responding. It is hypothesised that acquisition processes operate directly to increase the probability of attending to the relevant dimension of Phase-1, while extinction processes operate to decrease the probability of attending to the irrelevant dimension. The results from Experiments 1,2 and 3 serve to confirm this prediction for the case where two values on each of the relevant and irrelevant dimension are presented.

Honig and Urcuioli (1981) identify two distinct approaches to how attentional processes operate. "The first ascribes attentional effects to a selection among concurrently available stimuli....(and) the second is that attentiveness to all predictive stimuli is enhanced by discrimination training" (p.430). The first of these approaches characterises the selective attention theories discussed earlier (Lovejoy, 1968: Sutherland \& Mackintosh, 1971). The second approach refers to the general attentiveness theory proposed by Thomas. Thomas et a1. $(1969,1970)$ reported evidence to show that rather than a specific tendency to attend to only the particular stimulus differences of a stimulus dimension relevant during discrimination training, a general set to attend to stimulus differences is established to any potentially relevant stimuli.

Thomas et al. (1970) found discrimination training along one 
dimension sharpened the generalisation gradient along other dimensions which were either present but irrelevant, or, introduced after dimensional acquisition. (Irrelevant dimensions were only represented by one cue on that dimension). In a similar vein Robles et al. (1980) showed how an experimental group given initial discrimination training on a line tilt dimension exhibited better dimensional stimulus control by a second, wavelength dimension relative to a control group that had been exposed to the original tilt dimension, but who did not receive differential training. In Experiment 4 of the current series of experiments an ED-shift group was compared to a PD group that had been exposed to the original length dimension, but that had not received differential training. The ED group showed better dimensional stimulus control than the PD group. Therefore, the probability of attending to any dimension, even one that was irrelevant in Phase-1, is enhanced by prior differential training. That is, True-Discrimination on an unrelated dimension can enhance performance on a second dimension, consistent with the General Attentiveness theory of Thomas (1970).

Having established that a non-dimension specific decision rule may be learned as a result of discrimination training, we examined which factors determined the probability of attending to the Phase-2 relevant dimension in an ED shift. Two-process theories of discrimination learning (Lovejoy, 1968: Sutherland \& Mackintosh, 1971) and studies involving simultaneous discrimination learning (Esposito, 1975a: Ozioko \& May, 1977) identified the mode of presentation of the irrelevant dimension as critical in obtaining an ID-ED difference. 
146.

In a successive paradigm it is not possible to vary the dimensions within and between trials as Ozioko and May (1977) had done with adults and Dickerson et al. (1971) had done with children. However, Trabasso et a1. (1966) used a different method to compare children's shift learning in the presence and absence of an irrelevant dimension. In order to avoid the effects of novelty increasing the probability of responding to the extradimension in Phase-2, they held the irrelevant dimension of Phase-1 constant. There was no difference between mean error for ID and ED shift when the irrelevant dimension was held at a constant value over trials. Similarly, when the irrelevant dimension of Phase-1 in Experiment 5 was held at a constant (constant-irrelevant) value, the ID-ED shift difference was attenuated.

Of critical importance is the difference between cues on the dimensions. Experiment 2 established that it was the differences between cues rather than the specific values of those cues that was important. Where the irrelevant dimension of Phase-1 was ConstantIrrelevant, the subject did not learn about the dimension's irrelevance, and therefore in Phase-2 the new task was not impaired to the same extent as where the dimension was rendered explicitly irrelevant by random reinforcement of different values on that dimension (eg. Experiment 3).

Experiment 6 examined a more general question, one previously identified in the stimulus generalisation literature (Thomas, 1970: Thomas et al. 1970): cue utilisation in testing versus cue selection 
during training. Experiment 6 replicated Experiment 5, but with testing conducted on the orientation dimension only. With the exception of the CI-CI condition, the one-dimensional generalisation tests yielded the same pattern of results in Experiment 6 as did the two-dimensional tests in Experiment 5 . It can therefore be concluded that the possibility of masking did not alter the conclusions made in Experiment 5.

SUMMARY

Taken together the results of the present experiments indicate that in general, compared to ID shifts, ED shifts are slower because the Phase-1 relevant dimension must lose control in Phase-2, and the Phase-1 irrelevant dimension must gain control in Phase-2 (Experiment 3). However, the inverse relation between loss of control by one dimension and gaining of control by the other does not occur in a way consistent with the Inverse Hypothesis of theories of selective attention (Lovejoy, 1968: Sutherland \& Mackintosh, 1971). Firstly, one dimension loses control before the other starts gaining control (Experiment 3). Secondly, control by the previously relevant dimension does not directly compete with control by the new relevant dimension in Phase-2 (Experiment 4). If anything, the previously relevant (TD) dimension factilitates control by the new relevant dimension, consistent with attentional enhancement (Thomas, 1970).

In addition the development of control by the new relevant 
dimension in Phase-2 of an ED shift is retarded, relative to development of control by the relevant dimension in Phase-2 of an ID shift, by non-differential (PD) training on that dimension during Phase-1 (Experiment 5). This may be labelled a "learned irrelevance" effect.

\section{ATTENTION AS TWO-DIMENSIONAL STIMULUS CONTROL}

The use of operant conditioning techniques to investigate the ID-ED shift phenomenon has several advantages over trials to criterion methods and forms part of a trend to examine traditionally cognitive phenomena using operant methodology (Marr, 1984).

One advantage is that using generalisation tests exposes each subject to a range of test stimuli comprising a dimension rather than one or two discrete stimuli from which attention to a dimension is inferred. Gradient slope affords a direct measure of the extent of stimulus control by either dimension.

A second advantage is that dimensional control can be measured at different transitional stages during the Phase-2 discrimination. This is particularly appropriate to the ID-ED shift which is a transitional or transient phenomenon.

A third advantage in the current operant approach is that testing is carried out in extinction and involves a relatively large number of responses to stimuli on a dimension. In addition, all subjects were tested with an identical set of stimuli in which onty 
the random orders were changed. This last factor permitted an additional analysis of the ID-ED shift data described in the following section. The aim of the present approach is to estimate the magnitude of control by two dimensions in an ID-ED paradigm. Various models have been proposed (Blough, 1972: Chase \& Heinemann, 1972: Heinemann et al., 1969, 1970, 1973: Purtle \& Newman, 1969) to show how two dimensions combine to produce a response. In the present analysis of the role of attention in the performance of an ID-ED shift, it has been argued that instead of referring to attention in terms of a mediating response, attention is synonymous with dimensional stimulus control.

\section{QUANTIFICATION OF TWO-DIMENSIONAL STIMULUS CONTROL}

The two-dimensional gradients presented here were interpreted in terms of the extent of control by the length and orientation dimensions and the possible interaction between the dimensions. In most cases there was no significant interaction between the dimensions. Notwithstanding the ogival form of the gradients, the two-dimensional surfaces can be represented by two-dimension linear surfaces in which the extent of control by the length and orientation dimensions is given by the slopes of the marginal gradients. Thus the mean rating is given by a linear model in which $x_{1}$ and $x_{2}$ are the values on the length and orientation dimensions and $a_{1}$ and $a_{2}$ are coefficients that quantify the magnitude of control exerted by length and orientation. That is, for mean rating $y, y=a_{0}+a_{1} x_{1}+$ $a_{2} x_{2}$. In the analysis that follows, values of the coefficients were 
150.

TABLE 19.

Variance accounted for by the multiple linear regression analyses for the twenty conditions in Experiments 3 to 5 .

$\begin{array}{ll}\text { ID (0) } & 0.95 \\ \text { ID (8) } & 0.94 \\ \text { ID (12) } & 0.94 \\ \text { ID (16) } & 0.95 \\ \text { ID (20) } & 0.93 \\ \text { ED (0) } & 0.96 \\ \text { ED (8) } & 0.93 \\ \text { ED (12) } & 0.64 \\ \text { ED (16) } & 0.93 \\ \text { ED (20) } & 0.93 \\ & \\ \text { PD (12) } & 0.32 \\ \text { PD (20) } & 0.84 \\ \text { ED (12) } & 0.64 \\ \text { ED (20) } & 0.93 \\ & \\ C I-C I(12) & 0.87 \\ C I-C I(20) & 0.93 \\ \text { CI-2D (12) } & 0.94 \\ \text { CCI-2D (20) } & 0.96 \\ \text { 2D-CI (12) } & 0.92 \\ \text { 2D-CI (20) } & 0.93 \\ \text { 2D-2D (12) } & 0.71 \\ \text { 2D-2D (20) } & 0.94\end{array}$


found by multiple linear regression analyses. Coefficients of zero indicate no control by a dimension, and coefficients of 0.86 and above indicate maximal control. Table 19 lists the variance accounted for by the multiple linear regression analyses for the different conditions of the present experiment. In most cases these values were satisfactorily high.

In order for the coefficients to represent the extent of stimulus control by the two dimensions on the same scale, the seven stimulus values on length and orientation dimensions were placed on the same ordinal scale ( 1 to 7 ). Stimulus values 1 to 7 (orientation) represent $15^{\circ}$ through $75^{\circ}$ and stimulus values 1 to 7 (length) represent $8 \mathrm{~mm}$ through $20 \mathrm{~mm}$. Length and orientation gradients for post-extradimensional criterion performance are shown in Figure 26. These gradients were obtained by plotting response ratings on one dimension averaged over all values of the other dimensions. The gradients exhibited a very close fit. The ordinal scale ( 1 to 7 ) therefore dealt with the common scaling of the dimensions satisfactorily.

Figure 27 shows regression coefficients for line length $(x)$ and line orientation (o) plotted against trials of Phase-2 training for ID shift and ED shift in Experiment 3. The degree of control by each dimension is expressed in terms of a single regression coefficient. The ID-ED shift difference is given by the difference between coefficients for the relevant dimensions in the ID and ED conditions at any stage of Phase-2 training. In the ID shift, 
152.

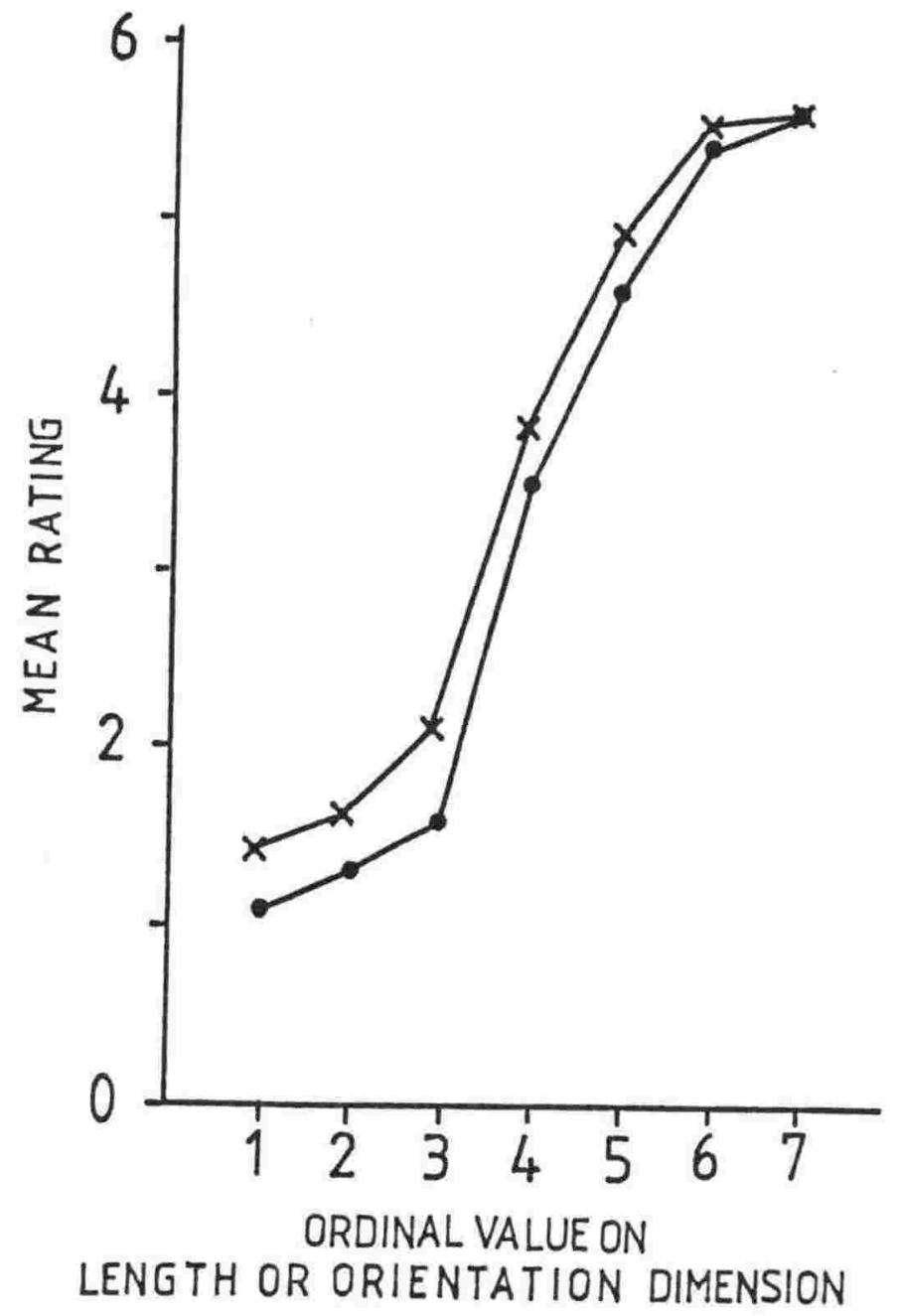

Figure 26: A comparison of response ratings to stimulus values on the dimensions of length $(x)$ and orientation (•) following Phase-2 ED (criterion) training. The rating scale has a minimum possible rating of one (1) and a maximum possible rating of $\operatorname{six}(6)$. 

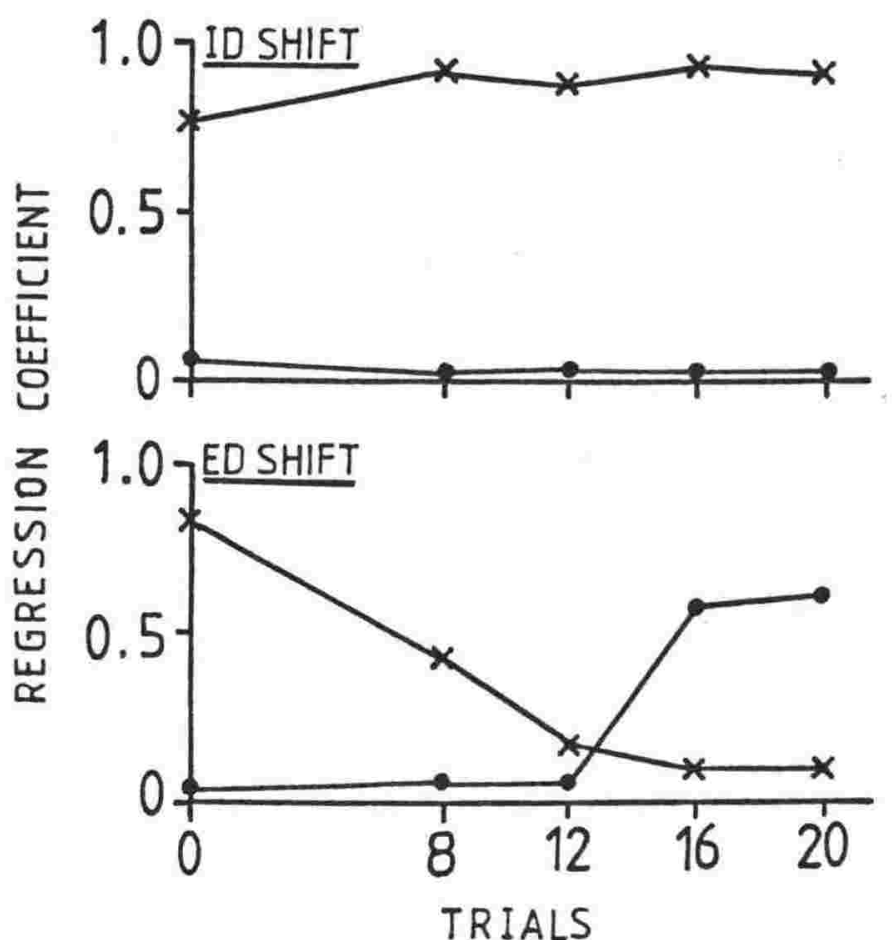

Figure 27: Regression coefficients for the length and orientation dimensions plotted against trials of Phase-2 ID or ED discrimination training in Experiment 3. [Length $(x)$ and Orientation $(\bullet)$ ] 
control by the relevant length dimension is consistently high between Phases 1 and 2, and for orientation, consistently low. For the ED shift, the influence of the previously relevant length dimension decreases, and control by the Phase-2 relevant dimension, orientation, increases. Thus the ID shift involves maintaining stimulus control that was established in Phase-1 throughout Phase-2. The ED shift, however, involves reversing control by the two dimensions between Phases 1 and 2. Figure 27 shows that the control established in Phase-1 persists through the first 12 trials of Phase-2, and is reversed appropriate to the new discrimination only after 16 or 20 trials of Phase-2.

Given that the two dimensions of length and orientation alone control responding, the Inverse Hypothesis would predict that as length lost control in Phase-2, orientation would correspondingly gain control at the same rate. However, Figure 27 shows that orientation does not start to gain control of responding until after trial 12 when length, the previously relevant dimension has established minimal control. This type of non-continuity effect would not be predicted by the Sutherland and Mackintosh (1971) model of discrimination learning.

Figure 28 shows the degree of control (regression coeffecients) exerted by both length and orientation dimensions over trials of ED training following either Pseudo (PD) or Extra (ED) dimensional discrimination training in Experiment 4. Two-process models based on the inverse hypothesis (Lovejoy, 1968: Sutherland \& Mackintosh, 
155.

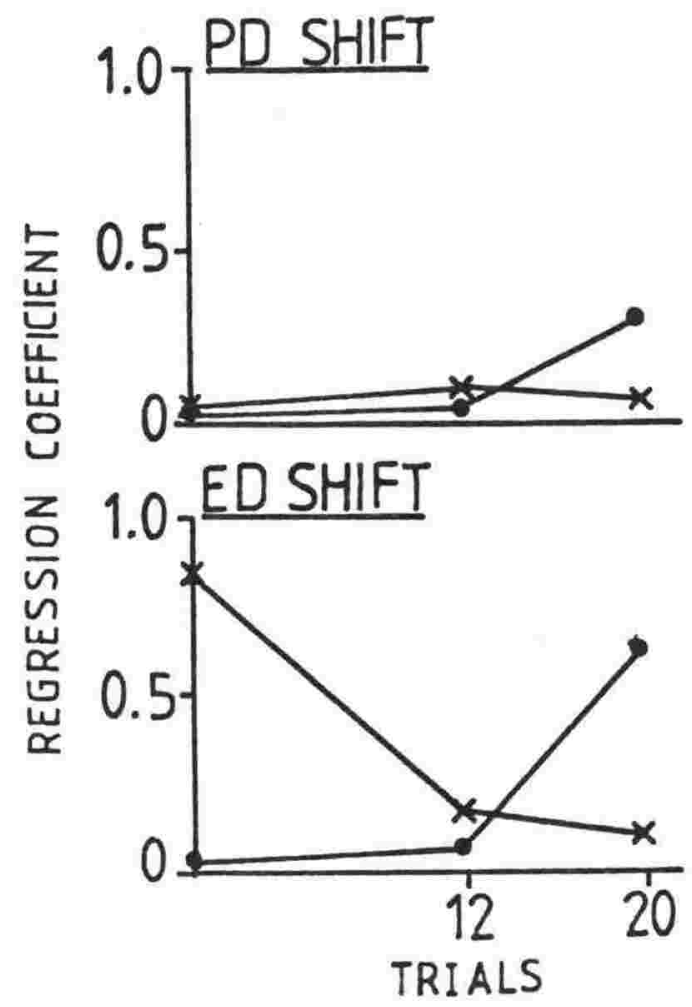

Figure 28: Regression coefficients for the length and orientation dimensions plotted against trials of Phase-2 discrimination training following Phase-1 Pseudo-discrimination (PD) or True-discrimination (ED) in Experiment 4. [Length $(x)$ and Orientation $(\bullet)$ ] 
156.

1971) would require that discrimination training on the length dimension in Phase-1 would impair control by orientation in Phase-2 relative to non-differential training on the length dimension in Phase-1. A general attentiveness model (Thomas, 1970) would make the opposite prediction: better control by orientation in Phase-2 because of previous discrimination training with length in Phase-1. The present data support the second model. Dimensional stimulus control and hence attention to orientation increases more rapidly following discrimination training on length relative to nondifferential training on length.

Results from Experiment 5 are shown in Figure 29. In Experiment 5 the relative influence of length discrimination in Phase-1 on the acquisition of the orientation discrimination in Phase-2 is tested with (2D) and without (CI) differences in orientation in Phase-1. Following Phase-1 training the index of control by length for all groups is high. In the CI-2D condition orientation acquires stronger control more rapidly than where it had two values in Phase-1 (2D-CI) and $(2 D-2 D)$.

The Chase and Heinemann (1972) Model of Two-Dimensional Stimulus Control

Chase and Heinemann (1972) described a quantitative model of two-dimensional stimulus control based on data from post-discrimination generalisation gradients. They obtained such data using conditional discrimination in a discrete trials procedure with pigeons. Such procedures produced the same sigmoidal gradients as 

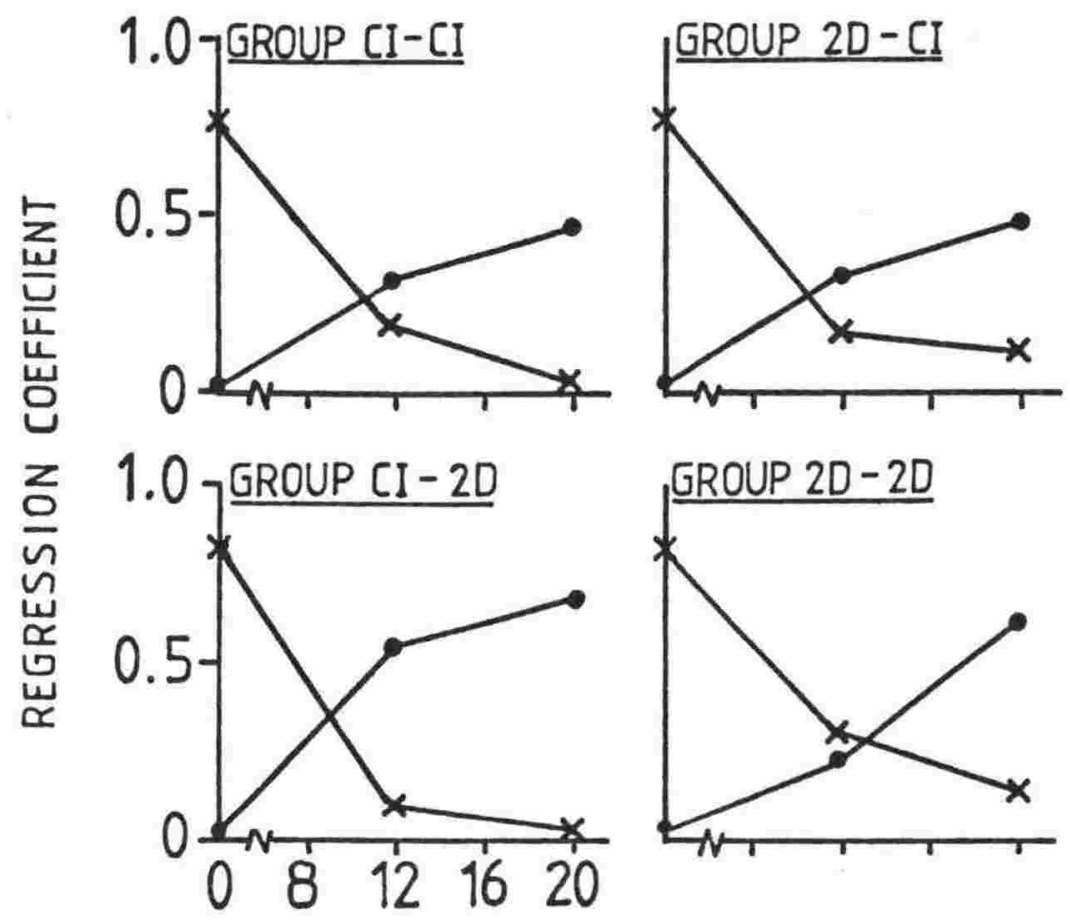

TRIALS

Figure 29: Regression coefficients for the length $(x)$ and orientation ( $\bullet$ ) dimensions plotted against trials of Phase-2 discrimination training for the four experimental conditions in Experiment 5. 
in the current series of experiments. The main area of interest in their work was the relative discriminability of two stimulus dimensions as it affected stimulus control, but their model has interesting implications for studying the effects of selective attention.

Chase and Heinemann (1972) argued that the degree to which behaviour is affected by stimulus changes along dimensions is determined by two factors: firstly, the decision rule learned by the subject, and secondly, the probabilities of attending to each dimension. The slope of the decision line, which is derived empirically from post discrimination generalisation gradients, is generated in the following way:

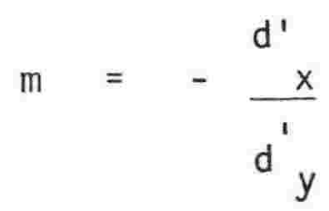

where $m$ is the slope of the decision line, and, $d$ ' is the difference between the means on dimension $x$ and $y$ when the variables of the two decision axes are expressed in standard deviation units. If one dimension is constant-irrelevant during training, then the optimal decision line is perpendicular to the axis of the other stimulus dimension.

Chase and Heinemann (1972) assess the relevance of the two factors hypothesised to affect the relative influence of stimulus variations along each dimension. These were the decision rule (as represented by the slope of the decision line) and the 
probability of attention to each dimension. Differences in relative influence of the two dimensions resulted primarily from differences in slope of the decision line (which determined the slopes of the two sets of choice curves) rather than differences in attention to the two dimensions. This confirms the conclusions drawn here that stimulus differences have a critical role in determining the effect of discrimination training. 
APPENDIX I 
160.

\section{TABLE 1}

Summaries of two-way analyses of variance to show the effect of Number of Trials of ID Phase-2 discrimination training as a function of either the relevant dimension, length $(x)$ or the irrelevant dimension, orientation $(\bullet)$.
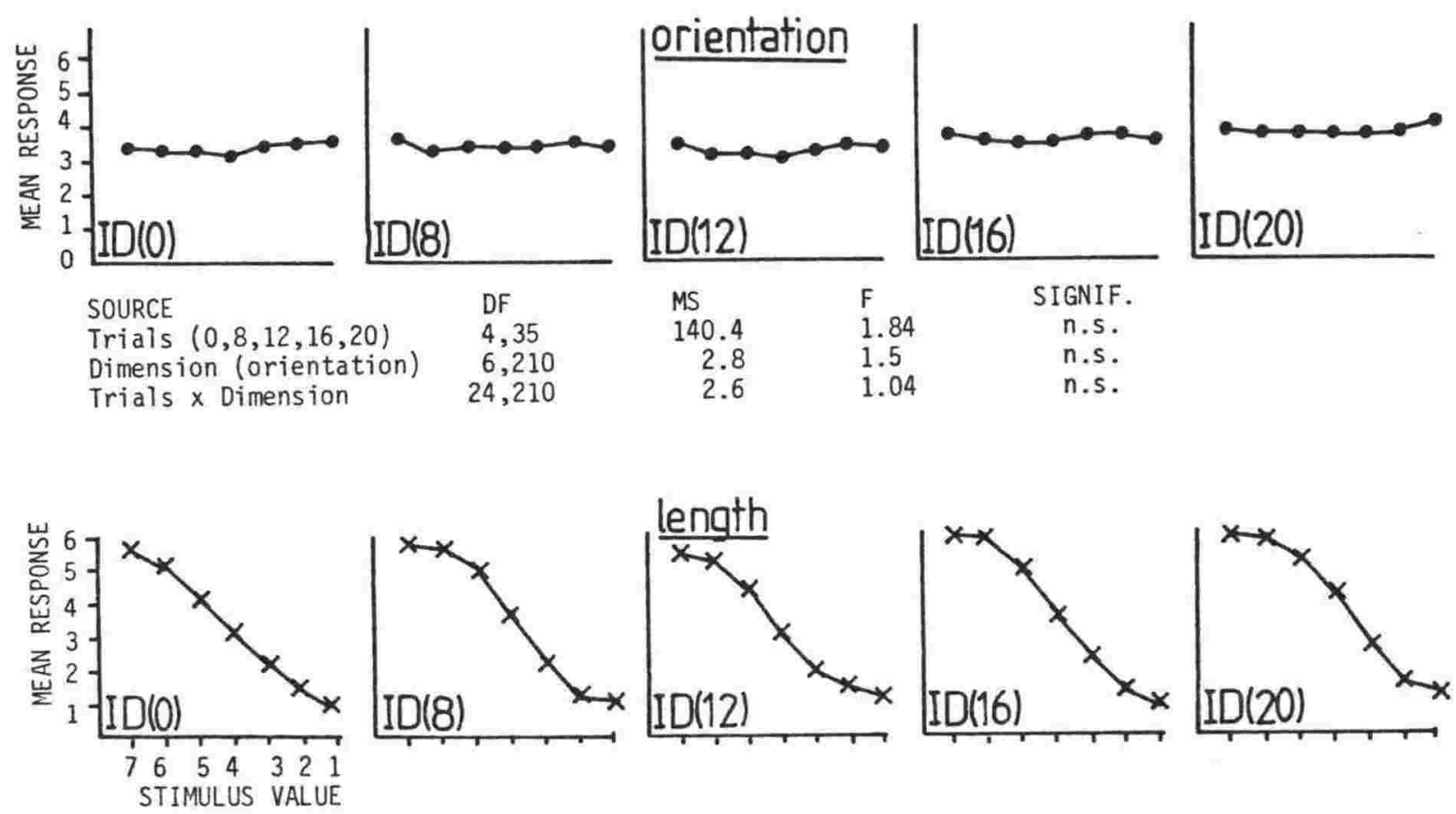

SOURCE

Trials $(0,8,12,16,20)$

Dimension (length)

DF

4,35

6,210

24,210

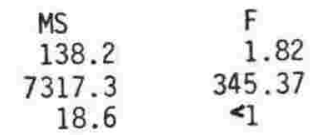

SIGNIF.

n.s.

p $<.001$

Trials $x$ Dimension

18.6

n.s. 


\section{TABLE 2}

Summaries of two-way analyses of variance to show the effect of Number of Trials of ED Phase-2 discrimination training as a function of either the relevant dimension, orientation ( ) or the irrelevant dimension, length $(x)$.
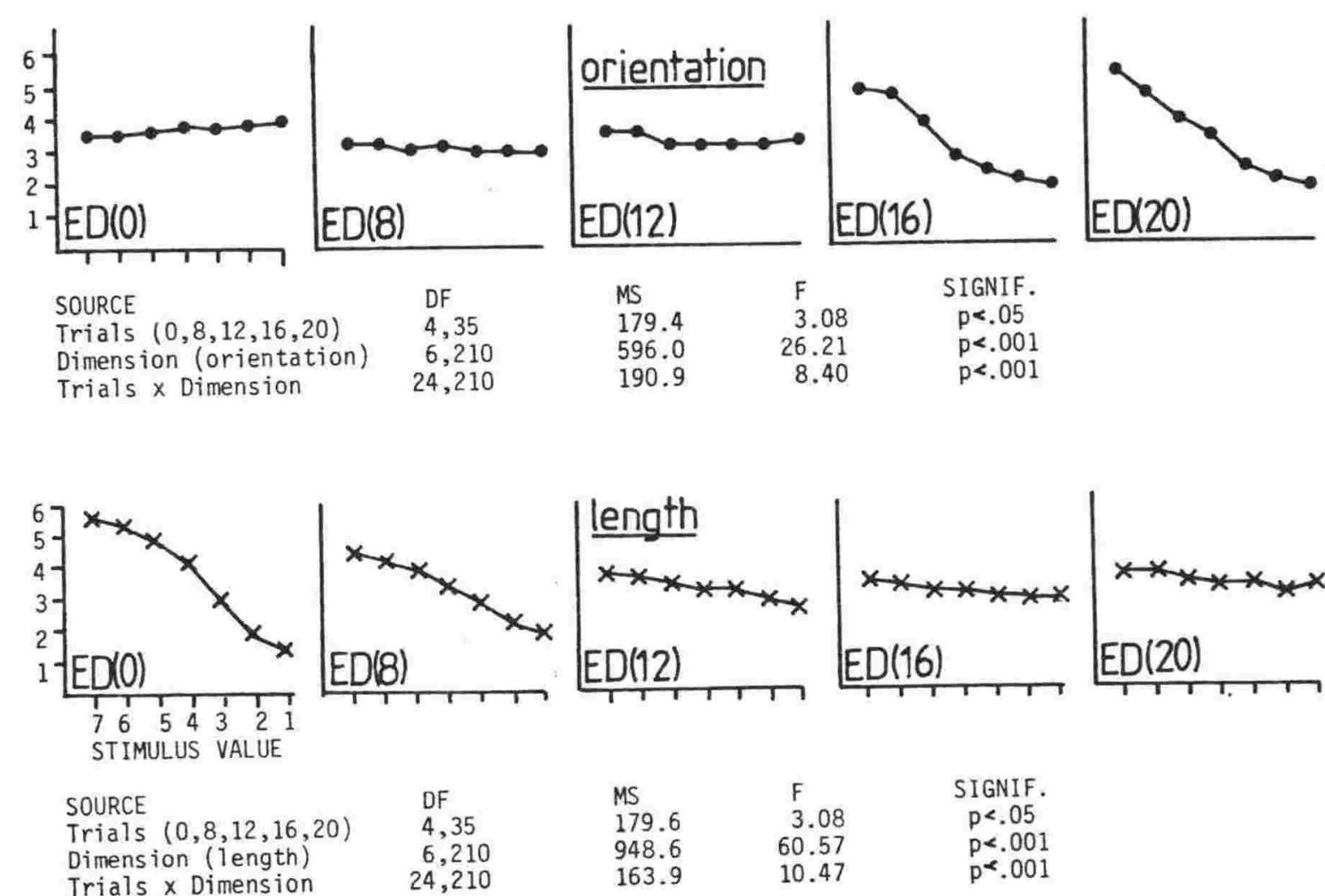

DF

4,35

6,210

24,210

MS

179.6

948.6

163.9
F

3.08

60.57

10.47
SIGNIF.

$p<.05$

$p<.001$

p $<001$ 


\section{TABLE 3}

Summary of two-way analyses of variance to show the between-group effect of Type of Phase-1 training (TD or PD) as a function of dimension, length ( $x$ ) or orientation ( ), collapsed over either 12 or 20 trials of Phase-2 discrimination training.
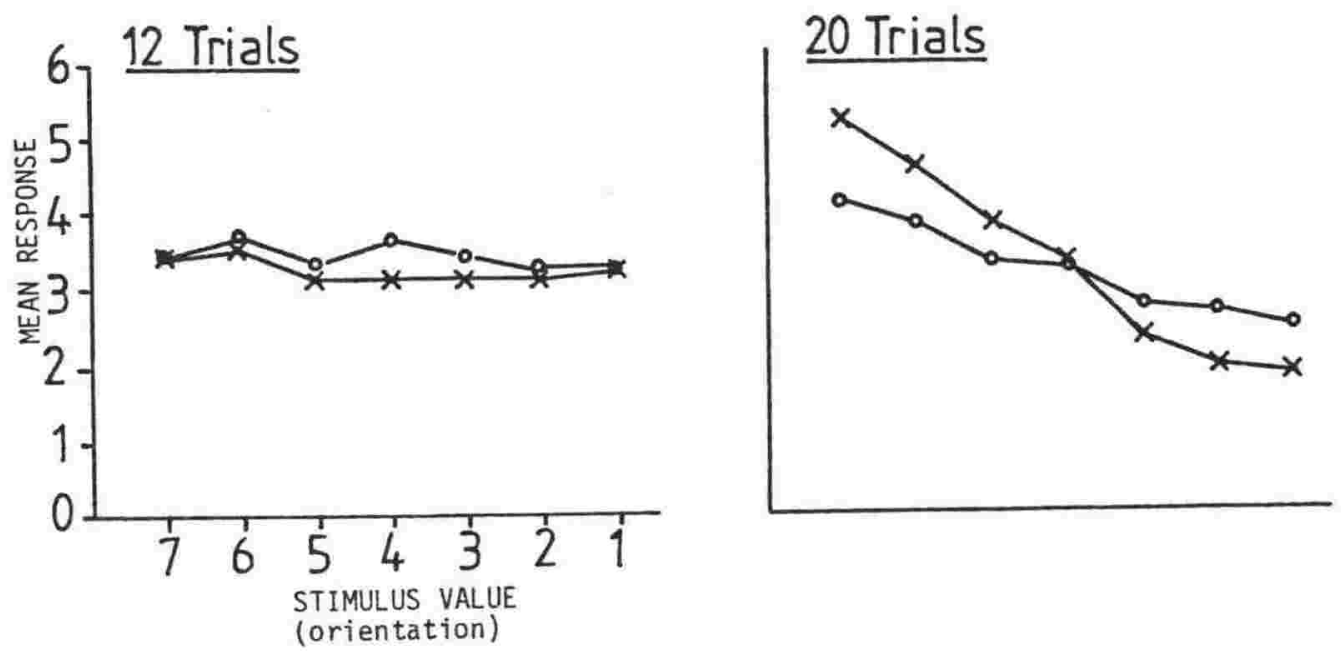

SOURCE

DF

MS

F SIG.

$\frac{12 \text { Trials }}{\text { Array }}$

Dimension

Array $x$ Dimension

1,14

6,84

6,84

0.6

$<1$

1.36

0.3
0.1

$<1$

n.s.

20 Trials

Array
Dimension

Array $x$ Dimension

1,14

6,84

0.2

15.7

1.9

27.6

3.38

n.s.

6,84

$p<.00$

$p<.01$ 


\section{TABLE 4}

Summary of two-way analyses of variance to show the between group effects of Type of Phase-1 stimulus Array (CI or 2D) as a function of dimension (orientation - $\bullet$ ), collapsed over either 12 or 20 trials of Phase-2 ED training with length (irrelevant) and orientation (relevant).
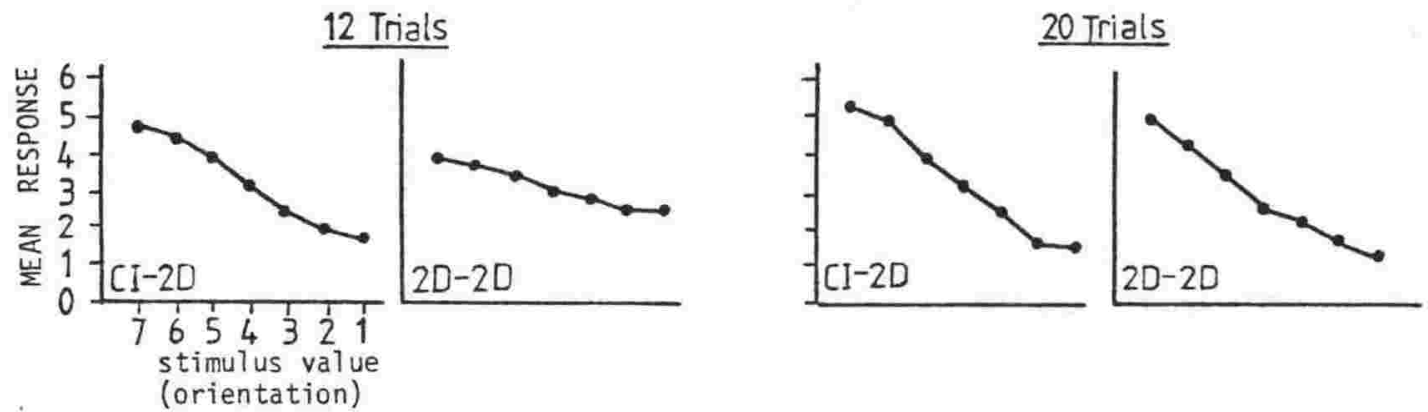

SOURCE

12 Trials

Array

Dimension

Array $x$ Dimension

$\frac{20 \text { Trials }}{\text { Array }}$

Dimension

Array $x$ Dimension

$\begin{array}{ccc}\text { DF } & \text { MS } & F \\ 1,14 & 0.15 & <1 \\ 6,84 & 11.9 & 17.97 \\ 6,84 & 1.7 & 2.61\end{array}$

$\begin{array}{rrc}1,14 & 3.0 & 1.46 \\ 6,84 & 31.3 & 69.5 \\ 6,84 & 0.2 & <1\end{array}$

SIGNIFICANCE

$$
\begin{aligned}
& \text { n.s. } \\
& p<.001 \\
& p<.05
\end{aligned}
$$

$$
\begin{gathered}
\text { n.s. } \\
\text { p } \\
\text { n. } 001 \\
\text {. }
\end{gathered}
$$




\section{TABLE 5}

Summary of two-way analyses of variance to show the between group effect of Type of Phase-2 stimulus Array (CI or 2D) as a function of dimension (orientation - $\bullet$ ) collapsed over either 12 or 20 trials of Phase-2 ED training with length (irrelevant) and orientation (relevant).
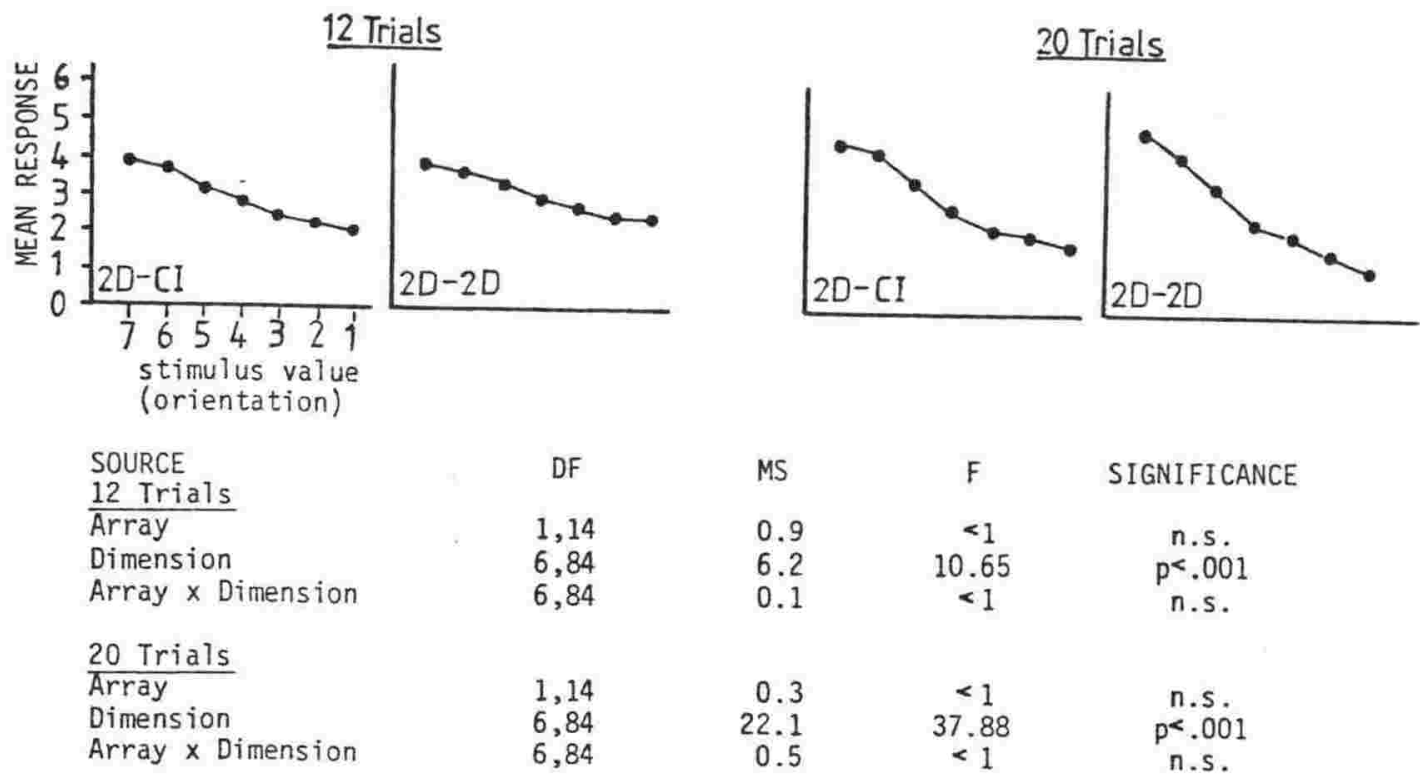

MS

0.9

6.2

0.1

$\mathrm{F}$

$<1$

$<1$

0.3

22.1
0.5

$<1$
37.88
$<1$
SIGNIFICANCE

$$
\begin{gathered}
\text { n.s. } \\
p<.001 \\
\text { n.s. }
\end{gathered}
$$

n.s. $p<.001$ 
165.

\section{TABLE 6}

Summary of two-way analyses of variance to show the between group effect of Type of Phase-1 stimulus Array (CI-2D) as a function of dimension (orientation - $\bullet$ ) collapsed over either 12 or 20 trials of Phase-2 ED training with length (irrelevant) and orientation (relevant).

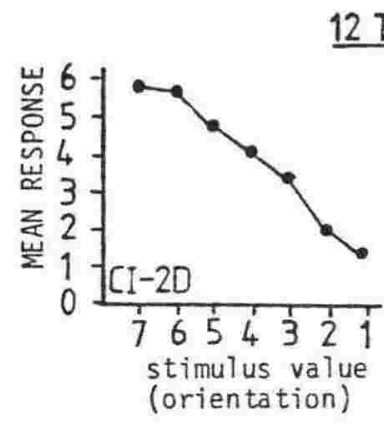

2 Trials

SOURCE

12 Trials

Dimension

Array $x$ Dimension
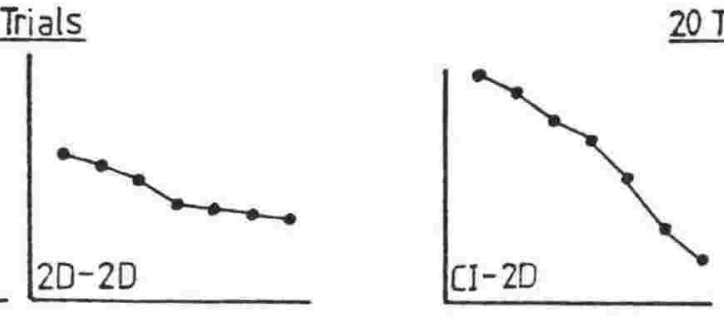

20 Trials

20 Trials

Dimension

DF

MS

$F$

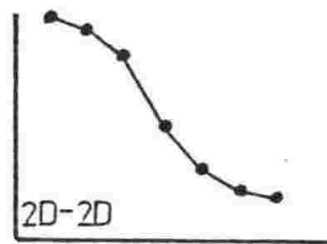

Array $x$ Dimension

1,14

6,84

22.6

22.5

6.23

6,84

5.2

31.8

7.34

SIGNIFICANCE

1,14
6,84
6,84

5.6

57.9

1.91

121.14

3.28

$p<.001$

$p<.001$

$p<.001$

n.s.

$p<.001$

$p<.01$ 


\section{TABLE 7}

Summary of two-way analyses of variance to show the between group effect of Type of Phase-2 stimulus Array (CI or 2D) as a function of dimension (orientation - $\bullet$ ) collapsed over either 12 or 20 trials of Phase-2 ED training with length (irrelevant) and orientation (relevant).
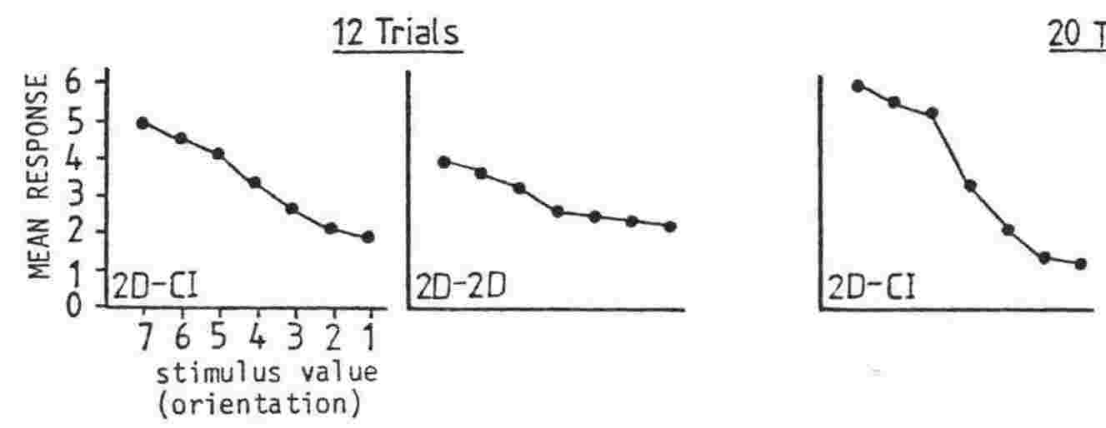

20 Trials

SOURCE

$\frac{12 \text { Trials }}{\text { Array }}$

Dimension

Array $\times$ Dimension

20 Trials

Dimension

Array $x$ Dimension
DF

1,14
6,84

6,84
6,84

1,14
6,84
6,84

\begin{abstract}
MS
\end{abstract}
5.6
13.7

1.3

0.2
65.9
0.05

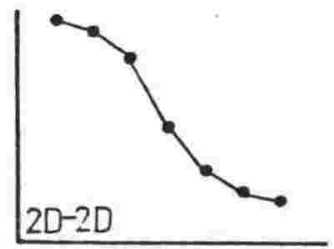

SIGNIFICANCE

1.16

20.76

1.9

pe.s.

n.s.

n.s.

p<.001

n.s. 
APPENDIX II 


\section{EXPERIMENT $2 \mathrm{~A}$}

METHOD

Subjects were 16 undergraduates who were randomly assigned to one of two experimental groups, with equal numbers of males and females in each group. The apparatus and general procedural conditions were the same as those used for the ID (R) shift group in Experiment 2.

Phase-1 discrimination training. For both groups the same discrete trials procedure was followed as for ID $(L)$ in Experiment 1 . Four training stimuli were presented equally often in a random order over 24 training trials. 'Yes' ratings were required to $20 \mathrm{~mm}$ lines and ' $\mathrm{no}$ ' ratings were required to $8 \mathrm{~mm} 1$ ines, independently of line orientation $\left(15^{\circ}\right.$ or $\left.75^{\circ}\right)$.

Phase-2 discrimination training followed Phase-1 training without a break in the procedure. The rule relating 'yes' and 'no' ratings to specific stimulus values was the same as for the ID (R) shift group in Experiment 2. 'Yes' ratings were required to $8 \mathrm{~mm}$ lines and 'no' ratings were required to $20 \mathrm{~mm}$ lines, independently of line orientation $\left(25^{\circ}\right.$ or $\left.65^{\circ}\right)$. There were two transfer conditions. Group ID $(R) 12$ received 12 trials of Phase-2 discrimination training while group ID (R) 20 received 20 trials of Phase-2 discrimination training. Generalisation testing. A generalisation test was administered directly following either 12 or 20 trials of Phase-2 discrimination training. The testing procedure was identical to that used in 
Experiment 2, and involved 98 trials during which all combinations of line length and orientation were presented.

\section{RESULTS}

Generalisation gradients were plotted in the same way as in Experiment 2. Figure 1 (Appendix II) shows the post-Phase-2 generalisation gradients for both ID (R) 12 and ID (R) 20 trials. Both gradients are sharp along the dimension that was relevant in Phase-2 (length) discrimination training, and flat along the dimension that was irrelevant (orientation). The mean ratings for these groups had maxima at the stimulus value requiring 'yes' ratings in Phase-2 $(8 \mathrm{~mm})$ and minima at the stimulus value requiring 'no' ratings $(20 \mathrm{~mm})$.

Comparing these two groups to their non-Reversal ID counterparts in Experiment 3, ID (12) and ID (20) (Figure 11), it can be seen that the ID (R) groups performed the reversal ID shift within the same number of trials of Phase-2 discrimination training as the ID groups. The ID (R) groups exhibit the same degree of dimensional stimulus control by the length dimension as the ID groups, except that control is by the new, reversed training stimuli. 
169.

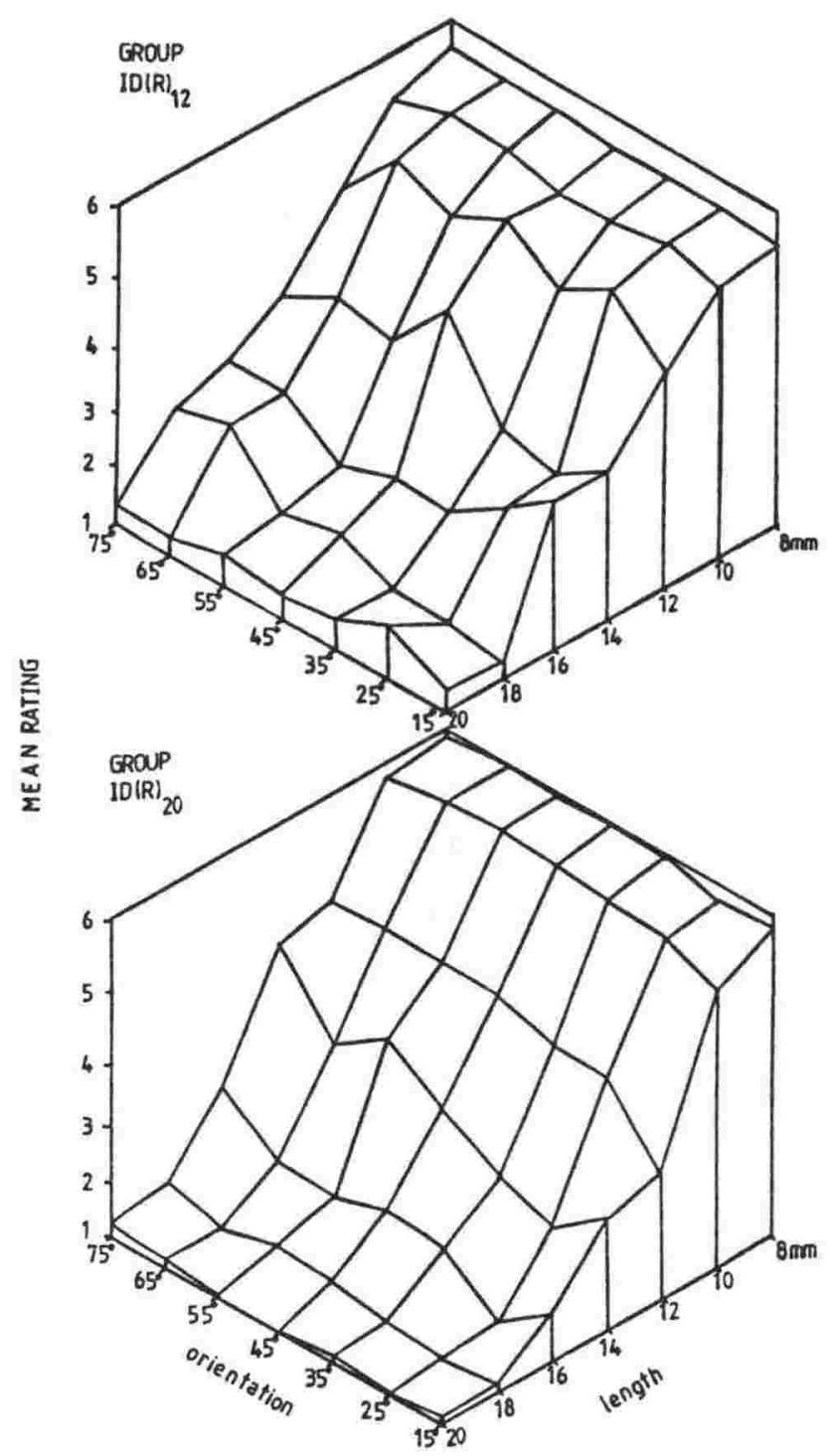

Figure 1 (Appendix II): Two-dimensional generalisation gradients following 12 or 20 trials of Phase-2 ID (Reversal) shift showing group mean response ratings as a function of the length and orientation dimensions, 
REFERENCES 


\section{REFERENCES}

ABRAHAM, F., GORMEZANO, I. and WIEHE, R. "Discrimination learning as a function of prior relevance of a partially reinforced dimension". Journal of Experimental Psychology, 1964, 67 (3), 242-9.

BLOUGH, D.S. "Recognition by the pigeon of stimuli varying in two dimensions". Journal of the Experimental Analysis of Behaviour, $1972, \underline{18},(3), 345-367$.

BUSS, A.H. "Rigidity as a function of reversal and non-reversal shifts in the learning of successive discriminations". Journal of Experimental Psychology, 1953, 45, (2), 75-81.

CAMPIONE, J.C. "Optional intradimensional and extradimensional shifts in children as a function of age". Journal of Experimental Psychology, 1970, 84, 296-300.

CHASE, S. and HEINEMANN, E.G. "Choices based on redundant information: an analysis of two-dimensional stimulus control". Journal of Experimental Psychology, 1972, 92, 161-175.

DICKERSON, D.J., WAGNER, J.F. and CAMPIONE, J. "Discrimination shift performance of kindergarten children as a function of variation of the irrelevant shift dimension". Developmental Psychology, 1970 3, (2), 229-235.

DOLL, T.J. and THOMAS, D.R. "Effects of discrimination training on stimulus generalisation for human subjects". Journal of Experimental Psychology, 1967, 75, 508-12.

EIMAS, P.D. "Comment: comparisons of reversal and non-reversal shifts". Psychonomic Science, 1965, $\underline{3}, 445-446$. 
EIMAS, P.D. "Effects of overtraining and age on intradimensional and extradimensional shifts in children". Journal of Experimental Child Psychology, 1966, $\underline{3}, 348-355$.

ESPOSITO, N.J. "Effects of stimulus novelty and dimensional saliency in human shift learning". Journal of Experimental Psychology, $1973, \underline{98},(2), 264-270$.

ESPOSITO, N.J. "Review of discrimination shift learning in young children". Psychological Bulletin, 1975a, 82, (3), 342-55.

ESPOSITO, N.J. "Modifiability of attention effects of dimensional salience and overtraining on discrimination shifts". Learning and Motivation, 1975b, $\underline{6}$, (1), 114-21.

EVANS, P.L.C. and HOGG, J. "Individual differences in the severely retarded child in acquisition, stimulus generalisation and extinction in go/no-go discrimination learning". Journal of Experimental Child Psychology, 1975, 20, 377-390.

FANTINO, E. and LOGAN, C.A. "The experimental analysis of behaviour: a biological perspective". W.H. Freeman and Company, San Francisco, 1979.

FISHER, M.A. and ZEAMAN, D. "An attention-retention theory of retardate discrimination learning" in: N.R. Ellis (Ed) International Review of Research in Mental Retardation. Academic Press, New York, No. 6, 1973.

FREEMAN, F. and THOMAS, D.R. "Attention vs. cue utilization in generalisation testing". Paper presented at the meeting of the Midwestern Psychological Association, Chicago, May 1967. 
GARNER, W.R. "The stimulus in information processing". American Psychologist, 1970, 25, 350-358.

GOODWIN, W.R. and LAWRENCE, D.H. "The functional independence of two discrimination habits associated with a constant stimulus situation". Journal of Comparative and Physiological Psychology, $1955,48,437-443$.

GORMEZANO, I. and GRANT, D.A. "Progressive ambiguity in the attainment of concepts on the Wisconsin Card Sorting Test". Journal of Experimental Psychology, 1958, 55, 621-627.

GRAY, V.A. and MACKINTOSH, N.J. "Control by an irrelevant stimulus in discrete-trial discrimination learning by pigeons". Bulletin of the Psychonomic Society, 1973, 1, (3), 193-195.

HALL, G. and CHANNELL, S, "A comparison of intradimensional and extradimensional shift learning in pigeons". Behavioural Processes, 1985, 10, 285-295.

HALL, G. and CHANNELL, S. "A search for perceptual differentiation produced by nondifferential reinforcement". Quarterly Journal of Experimental Psychology, 1980, 32, 185-95.

HARPUR, J.G.E. "Partial reinforcement and noticeability of an irrelevant dimension and their effects on subsequent performance in a concept-shift task". Psychological Reports, 1978, 42, 927-32.

HARROW, M. "Stimulus aspects responsible for the rapid acquisition of reversal shifts in concept formation". Journal of Experimental Psychology, 1964, 67, (4) 330-334. 
HARROW, M. and FRIEDMAN, G.B. "Comparing reversal and nonreversal shifts in concept formation with partial reinforcement controlled". Journal of Experimental Psychology, 1958, 55, 592-598.

heinemann, E.G., AVIN, E, SUlLivan, M.A. and CHASE, S. "Analysis of stimulus generalisation with a psychophysical method". Journal of Experimental Psychology, 1969, 80, 215-224.

HEINEMANN, E.G. and AVIN, E. "On the development of stimulus control". Journal of the Experimental Analysis of Behaviour, 1973, 20, (2), 183-195.

HEINEMANN, E.G. and CHASE, S. "Conditional stimulus control". Journal of Experimental Psychology, 1970, 84, 187-197.

HOGG, J. and EVANS, P.L.C. "Stimulus generalisation following extradimensional training in educationally subnormal (severely) children". British Journal of Psychology, 1975, 66, (2) 211-224.

HOGG, J. and EVANS, P.L.C. "Stimulus generalisation following extradimensional and intradimensional training in educable retarded children". Journal of Experimental Child Psychology, $1978,25,224-41$.

HONIG, W.K. "Attentional factors governing the slope of the generalisation gradient". In: R.M. Gilbert and N.S. Sutherland (Eds.) Animal discrimination learning, London: Academic Press, 1969.

HONIG, W.K. "Attention and the modulation of stimulus control". In: D. Mostofsky (Ed.) Attention: contemporary theory and analysis. New York: Appleton-Century-Crofts, 1970. 
HONIG, W.K. and URCUIOLI, P.J. "The legacy of Guttman and Kalish (1956): 25 years of research on stimulus generalisation". Journal of the Experimental Analysis of Behaviour, 1981, 36, (3) 405-445.

HOWARD, R.W. "Stimulus generalisation along a dimension based on a verbal concept". Journal of the Experimental Analysis of Behaviour, 1979, 32, 199-122.

ISAACS, I.D. and DUNCAN, C.P. "Reversal and nonreversal shifts within and between dimensions in concept formation". Journal of Experimental Psychology, 1962, 64, (6), 580-585.

JOHNSON, D.F. "Determiners of selective stimulus control in the pigeon". Journal of Comparative and Physiological Psychology, 1970, 70, (2), 298-307.

JOHNSON, P.J. "Factors affecting transfer in concept identification problems". Journal of Experimental Psychology, 1966, 72, (5) 655-660.

KEMLER, D.G. and SHEPP, B.E. "Learning and transfer of dimensional relevance and irrelevance in children". Journal of Experimental Psychology, 1971, 90, (1), 120-7.

KENDLER, H.H. and D'AMATO, M.J. "A comparison of reversal and nonreversal shifts in human concept formation and behaviour". Journal of Experimental Psychology, 1955, 49, 165-174.

KENDLER, H.H. and KENDLER, T.S. "Vertical and horizontal processes in problem solving". Psychological Review, 1962, 69, 1-16. 
KENNEDY, T.D. and GERSTEN, C.D. "Comparison of intradimensional and extradimensional shifts using geometric and symbolic stimuli". Bulletin of the Psychonomic Society, 1976, $\underline{7},(5), 458-60$.

KEPPEL, G. "Design and Analysis: A researcher's handbook" (2nd ed.), New Jersey: Prentice-Hall Inc. 1982.

KRECHEVSKY, I. "Hypotheses in rats". Psychological Review, 1932 , 39, 516-532.

LASHLEY, K.S. "Brain mechanisms and intelligence". Chicago, University of Chicago Press, 1929.

LASHLEY, K.S. "An examination of the continuity theory as applied to discriminative learning". Journal of General Psychology, 1942 , 26, 241-265.

LAWRENCE, D.H. "Acquired distinctiveness of cues: I. Transfer between discriminations on the basis of familiarity with the stimulus". Journal of Experimental Psychology, 1949, 39, 770-784.

LAWRENCE, D.H. "Acquired distinctiveness of cues: II. Selective association in a constant stimulus situation". Journal of Experimental Psychology, 1950, 40, 175-188.

LEBOW, M.D. and TRITT, J. "Intradimensional and extradimensional shifts using a total change design with word stimuli". Psychonomic Science, 1971, 22, (6), 325-27. 
LOGAN, F.A. and FERRARO, D.P. "Systematic analyses of learning and motivation". New York, John Wiley \& Sons, 1978.

LOVEJOY, E. "Analysis of the overlearning reversal effect". Psychological Review, 1966, 73, 87-103.

LOVEJOY, E. "Attention in discrimination learning". San Francisco: Holden-Day, 1968.

LOWENKRON, B. "Performance in intra- and extra-dimensional shifts as a function of solution mode in concept-identification problems". Psychological Reports, 1975, 37, 447-454.

MACKINTOSH, N.J. "Selective attention in animal discrimination learning". Psychological Bulletin, 1965, 64, 124-150.

MACKINTOSH, N.J. "Attention and probability learning" in: D.I. Mostofsky (Ed.) Attention: contemporary theory and analysis. New York, Appleton-Century-Crofts, 1970, 173-191.

MACKINTOSH, N.J. "Stimulus selection: learning to ignore stimuli that predict no change in reinforcement" in: Hinde, R.A. \& Stevenson-Hind, J. (Eds.) Constraints on learning: limitations and predispositions. New York, Academic Press, 1973, 75-100.

MACKINTOSH, N.J. "The psychology of animal learning". Academic Press, London, 1974. 
MACKINTOSH, N.J. "Stimulus control: attentional factors" in:

W.K. Honig and J.E.R. Staddon (Eds.) Handbook of Operant

Behaviour. N.J., Prentice-Ha11, 1977, 481-513.

MARR, M.J. "Conceptual approaches and issues". Journal of the Experimental Analysis of Behaviour, 1984, 42, (3), 353-362.

MEDIN, D.L. "Theories of discrimination learning and learning set" in: W.K. Estes (Ed.) Handbook of learning and Cognitive Processes. Volume 3. Approaches to human learning and motivation. Lawrence Erlbaum Assoc. Inc., N.J., 1976, 131-169.

NEHRKE, M.F. "Age and sex differences in discrimination learning and transfer of training". Journal of Gerontology, 1973, 28, (3) 320-27.

NEWLIN, R.J., RODGERS, J.P. and THOMAS, D.R. "Two determinants of the peak shift in human voluntary stimulus generalisation". Perception and psychophysics, 1979, 25, 478-86

NEWMAN, F.L. and BARON, M.R. "Stimulus generalisation along the dimension of angularity". Journal of Comparative and Physiological Psychology, 1965, 60, 59-63.

OZIOKO, J.O.C. and MAY, R.B. "Dimensional dominance and adult shift learning". Bulletin of the Psychonomic Society, 1977, $\underline{9}$ (4) $314-16$.

PURTLE, R.B. and NEWMAN, F.L. "Multidimensional stimulus generalisation of a tactile response along the dimensions of angularity and texture". Journal of Experimental Psychology, 1969, 81, 566-571. 
RAY, B.A. "Strategy in studies of attention: a commentary on D.I. Mostofsky"s Attention: Contemporary Theory and Analysis". Journal of the Experimental Analysis of Behaviour, 1972, 17, (2), 293-8.

RAY, B.A. and SIDMAN, M. "Reinforcement schedules and stimulus control" in: W.N. Schoenfeld (Ed.) The Theory of Reinforcement Schedules. New York, Appleton-Century-Crofts, 1970, 187-214.

ROBLES, L., NEWLIN, R.J. and THOMAS, D.R. "Potentiation of attentional enhancement in the pigeon produced by a 'blocking' stimulus". Animal Learning and Behaviour, 1980, $\underline{8}, 31-36$.

SHANAB, M.E. and YASIN, A.B. "Intradimensional and extradimensional shifts by Jordanian students". The Journal of General Psychology, $1979,100,199-213$.

SHEPP, B.E. and EIMAS, P.D. "Intradimensional and extradimensional shifts in the rat". Journal of Comparative and Physiological Psychology, 1964, 57, 357-61.

SHEPP, B.E. and GRAY, V.A. "Some effects of variable-within and variable-between irrelevant stimuli on dimensional learning and transfer". Journal of Experimental Psychology, 1971, 89, (1), 32-9.

SHEPP, B.E. and TURRISI, F.D. "Learning and transfer of mediating responses in discriminative learning" in: N.R. Ellis (Ed.) International Review of Research in Mental Retardation. Volume 2, New York, Academic Press, 1966, 85-121.

SIEGEL, S. "Overtraining and transfer processes". Journal of Comparative and Physiological Psychology, 1967, 64, 471-477. 
SINGH, N.N. "Attentional responses during discrimination learning by retarded children". PhD Thesis, Auckland University, 1978.

SINGH, N.N. and BEALE, I.L. "Attentional changes during discrimination learning by retarded children". Journal of the Experimental Analysis of Behaviour, 1978, 29, (3), 527-533.

SKINNER, B.F. "Science and human behaviour" New York: Macmillan, 1953.

SLAMECKA, N.J. "A methodological analysis of shift paradigms in human discrimination learning". Psychological Bulletin, 1968, 69, (6), 423-38.

SLAMECKA, N.J. "An improved discrimination-shift design". Psychonomic Science, 1969, 17, (2), 89.

SPENCE, K.W. "The nature of discrimination learning in animals". Psychological Review, 1936, 43, 427-449.

SUTHERLAND, N.S. "Visual discrimination in animals". British Medical Bulletin, 1964, 20, (2), 54-9.

SUTHERLAND, N.S. and MACKINTOSH, N.J. "Mechanisms of animal discrimination learning". Academic Press, New York, 1971.

SWITALSKI, R.W., LYONS, J. and THOMAS, D.R. "Effects of interdimensional training on stimulus generalisation". Journal of Experimental Psychology, 1966, 72, 661-666. 
180.

THOMAS, D.R. "The use of operant conditioning techniques to investigate perceptual processes in animals". In: R.M.

Gilbert and N.S. Sutherland (Eds.) Animal Discrimination

Learning. London: Academic Press, 1969.

THOMAS, D.R. "Stimulus selection, attention and related matters".

In: J.H. Reynierse (Ed.) Current issues in animal learning.

Lincoln, University of Nebraska Press, 1970, 311-356.

THOMAS, D.R., FREEMAN, F., SVINICKI, J.G., BURR, D.E.S. and LYONS, J. "Effects of extradimensional training on stimulus generalisation". Journal of Experimental Psychology, Monograph, 1970, 83, (1), 1-21.

THOMAS, D.R., SVINICKI, M.D. and VOGT, J. "Adaptation-level as a factor in human discrimination learning and stimulus generalisation". Journal of Experimental Psychology, 1973, 97, 210-219.

THOMAS, D.R. and VOGT, M.B. "Stimulus generalisation viewed from a signal detection perspective". New Zealand Journal of Psychology, $1983,12,(1), 1-6$.

TIGHE, T.J., GLICK, J. and COLE, M. "Subproblem analys is of discrimination-shift learning". Psychonomic Science, 1971, 24, 159-160.

TRABASSO, T. and BOWER, G.H. "Attention in learning: theory and research". New York, Wiley, 1968.

TRABASSO, T., DEUTSCH, J.A. and GELMAN, R. "Attention in discrimination learning of young children". Journal of Experimental Child Psychology, 1966, 4, 9-19. 
TURNER, C. and MACKINTOSH, N.J. "Stimulus selection and irrelevant stimuli in discrimination learning by pigeons". Journal of Comparative and Physiological Psychology, 1972, 78, (1), 1-9.

UHL, N. "Intradimensional and extradimensional shifts as a function of amount of training and similarity between training and shift stimuli". Journal of Experimental Psychology, 1966, 72, (3), 429-433.

WATSON, G.W. "Discrimination-shift behaviour as a function of rule learning and the number of irrelevant categories". Bulletin of the Psychonomic Society, 1975, 5 , (1), 49-50.

WHITE, K.G. and THOMAS, D.R. "Postdiscrimination stimulus generalisation in humans: An extension of Galizio and Brown". Animal Learning and Behaviour, 1979, $\underline{7}$, (14), 564-65.

WINER, B.J. "Statistical principles in experimental design". New York: McGraw-Hi11, 1962.

WINER, B.J. "Statistical principles in experimental design". (2nd ed.) New York: McGraw-Hi11, 1971.

WOLFF, J.L. "Concept-shift and discrimination reversal learning in humans". Psychological Bulletin, 1967, 68, 369-408.

WYCKOFF, L.B. "The role of observing responses in discrimination learning: Part I. Psychological Review, 1952, 59, 431-442.

ZEAMAN, D. and HOUSE, B.J. "The role of attention in retardate discrimination learning". In: N.R. Ellis (Ed.) Handbook of mental deficiency: psychological theory and research. New York: McGraw-Hi11, 1963. 